\title{
Real-Time Autonomous Obstacle Avoidance for Low-Altitude Fixed-Wing Aircraft
}

\author{
by
}

\author{
Shahaboddin Owlia
}

\author{
A Thesis submitted to \\ the Faculty of Graduate Studies and Research \\ in partial fulfilment of \\ the requirements for the degree of \\ Master of Applied Science \\ in
}

\author{
Mechanical Engineering \\ Carleton University \\ Ottawa, Ontario, Canada \\ January 2013 \\ Copyright (C) \\ 2013 - Shahaboddin Owlia
}


Library and Archives

Canada

Published Heritage

Branch

395 Wellington Street

Ottawa ON K1A ON4

Canada
Bibliothèque et

Archives Canada

Direction du

Patrimoine de l'édition

395 , rue Wellington

Ottawa ON K1A ON4

Canada
Your file Votre référence

ISBN: 978-0-494-94276-5

Our file Notre référence

ISBN: 978-0-494-94276-5
NOTICE:

The author has granted a nonexclusive license allowing Library and Archives Canada to reproduce, publish, archive, preserve, conserve, communicate to the public by telecommunication or on the Internet, loan, distrbute and sell theses worldwide, for commercial or noncommercial purposes, in microform, paper, electronic and/or any other formats.

The author retains copyright ownership and moral rights in this thesis. Neither the thesis nor substantial extracts from it may be printed or otherwise reproduced without the author's permission.
AVIS:

L'auteur a accordé une licence non exclusive permettant à la Bibliothèque et Archives Canada de reproduire, publier, archiver, sauvegarder, conserver, transmettre au public par télécommunication ou par l'Internet, prêter, distribuer et vendre des thèses partout dans le monde, à des fins commerciales ou autres, sur support microforme, papier, électronique et/ou autres formats.

L'auteur conserve la propriété du droit d'auteur et des droits moraux qui protege cette thèse. $\mathrm{Ni}$ la thèse ni des extraits substantiels de celle-ci ne doivent être imprimés ou autrement reproduits sans son autorisation.
In compliance with the Canadian Privacy Act some supporting forms may have been removed from this thesis.

While these forms may be included in the document page count, their removal does not represent any loss of content from the thesis.
Conformément à la loi canadienne sur la protection de la vie privée, quelques formulaires secondaires ont été enlevés de cette thèse.

Bien que ces formulaires aient inclus dans la pagination, il n'y aura aucun contenu manquant. 


\section{Abstract}

The GeoSurv II is an Unmanned Aerial Vehicle (UAV) being developed by Carleton University and Sander Geophysics. This thesis is in support of the GeoSurv II project.

The objective of the GeoSurv II project is to create a fully autonomous UAV capable of performing geophysical surveys. In order to achieve this level of autonomy, the UAV, which due to the nature of its surveys flies at low altitude, must be able to avoid potential obstacles such as trees, powerlines, telecommunication towers, etc. Developing a method to avoid these obstacles is the objective of this thesis.

The literature is rich in methods for trajectory planning and mid-air collision avoidance with other aircraft. In contrast, in this thesis, a method for avoiding static obstacles that are not known a priori is developed.

The potential flow theory and panel method are borrowed from fluid mechanics and are employed to generate evasive maneuvers when obstacles are encountered. By means of appropriate modelling of obstacles, the aircraft's constraints are taken into account such that the evasive maneuvers are feasible for the UAV. Moreover, the method is developed with consideration of the limitations of obstacle detection in GeoSurv II.

Due to the unavailability of the GeoSurv II aircraft, and the lack of a complete model for GeoSurv II, the method developed is implemented on the non-linear model of the Aerosonde UAV. The Aerosonde model is then subjected to various obstacle scenarios and it is seen that the UAV successfully avoids the obstacles. 
To my beloved mother who sacrificed her personal life to overcome the challenges our family encountered so that her children could succeed in life. 
No bird ever flew nonstop from New York to Tokyo, or raced 15 miles high at triple the speed of sound. But birds do something else. They do not conquer the air; they romance it.

Peter Garrison 


\section{Acknowledgments}

First I would like to thank my supervisor Professor Anton de Ruiter for his guidance, assistance and encouragement. In several occasions where I was under a lot of pressure he encouraged me to push harder, which ultimately led me to succeed in the task at hand. I would also like to thank him for his in-depth review of my thesis, which greatly contributed to the improvement of this document.

I would also like to express my gratitude to the GeoSurv II project manager, Professor Paul V. Straznicky, and Sander Geophysics for their continued support of the project.

Finally, I would like to especially thank Tom Hastie from CMC Electronics Esterline, for the help he gave me over the course of my master's degree. Tom taught me to fly R/C aircraft and greatly helped me in the development of ATB 10. Many times he would spend an excessive amount of time answering various questions I had regarding aircraft flight and avionics. Learning from a pilot and avionics engineer was a great opportunity for me that I cherish. By knowing and working with Tom, his passion of flying and airplanes was conveyed to me. 


\section{Table of Contents}

$\begin{array}{ll}\text { Abstract } & \text { ii }\end{array}$

Acknowledgments $\quad$ v

Table of Contents vi vi

List of Tables $\quad$ x

List of Figures $\quad$ xi

List of Acronyms xvi

1 Introduction $\quad 1$

1.1 GeoSurv II Project . . . . . . . . . . . . . . . . . 2

1.1 .1 Survey Path . . . . . . . . . . . . . . 3

1.1 .2 Potential Obstacles ................. 3

1.1.3 Obstacle Detection ................ 6

1.2 Thesis ........................... 7

1.2.1 Thesis Motivation . . . . . . . . . . . 7

1.2.2 Problem Statement and Thesis Structure . . . . . . . 8

2 Background Information and Method Selection 10

2.1 Literature Review . . . . . . . . . . . . . . . . . . . . 12 
2.1 .1 Road Map Method . . . . . . . . . . . . . . . 12

2.1.2 Rapidly Exploring Random Tree . . . . . . . . . . . . 14

2.1.3 Artificial Potential Fields . . . . . . . . . . . . 16

2.1.4 Harmonic Potential Functions . . . . . . . . . . 17

2.1 .5 Model Predictive Control . . . . . . . . . . . . 18

2.2 Method Selection . . . . . . . . . . . . . 19

3 Theory and Initial Results 22

3.1 Artificial Potential Field . . . . . . . . . . . . . . . 22

3.2 Harmonic Functions . . . . . . . . . . . . . 26

3.3 Potential Flow . . . . . . . . . . . . . . . . . . 28

3.3.1 Obstacle Avoidance using Potential Flow . . . . . . . . . . 29

3.3 .2 Flow primitives ................... 31

3.3 .2 .1 Uniform Flow . . . . . . . . . . . . 31

3.3 .2 .2 Sinks and Sources . . . . . . . . . 32

3.4 Panel Method . . . . . . . . . . . . . . . . . 34

3.4.1 Calculating $I_{i, j}, J_{x, j}, J_{y, j} \ldots \ldots \ldots \ldots$. . . . . 41

3.5 Obstacle Modeling \& Implementation . . . . . . . . . . . . . . 44

3.5.1 Obstacle Modeling . . . . . . . . . . . . . . 44

3.5.1.1 Increasing the Number of Panels . . . . . . . . 45

3.5.2 Incorporating Aircraft Constraints . . . . . . . . . 50

3.5.2.1 Aircraft Constraints ... . . . . . . . 50

3.5.2.2 Integration of OA and Aircraft Constraints . . . . 53

3.5.3 Multiple Obstacles . . . . . . . . . . . . . . 58

3.5 .4 Adding a Sink . . . . . . . . . . . . . 6 60

3.5.4.1 Computing the Required Sink Strength . . . . . . . 63

3.6 Summary . . . . . . . . . . . . . . . . 63 
4 Aircraft Autonomous Flight \& Simulation Framework 65

4.1 Simulation Framework . . . . . . . . . . . . . . 65

4.2 Autonomous Flight Architecture . . . . . . . . . . . . . 66

4.2.1 Autopilot Architecture . . . . . . . . . . . . 69

4.3 Autonomous Flight Architecture with Obstacle Avoidance . . . . . . 73

4.3.1 Modified Autopilot Architecture for OA . . . . . . . 74

5 Obstacle Avoidance Implementation \& Results 81

5.1 Obstacle Avoidance Implementation . . . . . . . . . . . . . . . 81

5.1 .1 Obstacle Consideration . . . . . . . . . . . 81

5.1.1.1 Top Plane Obstacle Consideration . . . . . . . . 82

5.1.1.2 Side Plane Obstacle Consideration . . . . . . . . 87

5.1 .2 Deliberator ... . . . . . . . . . . . 9 90

5.1 .3 Selector ......................... 91

5.1.4 Setting OA Parameters . . . . . . . . . . . 93

5.1.4.1 Calculating the Required Wedge Length, $h \ldots . . .95$

5.2 Obstacle Avoidance Results . . . . . . . . . . . . . . . 95

5.2.1 Fly-around Maneuver . . . . . . . . . . . . . . . . . 97

5.2 .2 Fly-over Maneuver . . . . . . . . . . . . . 101

5.2 .3 Multiple Obstacles ................. 103

5.3 Summary . . . . . . . . . . . . . . . . 106

6 Autonomous Aircraft Test Bed 109

6.1 ATB $10 \ldots \ldots \ldots \ldots \ldots$

6.1 .1 Senior Telemaster . . . . . . . . . . . . 109

6.1 .2 Autopilot ..................... 110

6.1 .3 Telemetry . . . . . . . . . . . . . . 112

6.2 Flight Tests ......................... 114 
6.3 Obstacle Avoidance Flight Test . . . . . . . . . . . . . . . . 117

7 Conclusions $\quad 119$

7.1 Contributions . . . . . . . . . . . . . . . . 120

7.2 Future Work . . . . . . . . . . . . . . . . 121

$\begin{array}{ll}\text { List of References } & 122\end{array}$

$\begin{array}{ll}\text { Appendix A The } \delta \text {-Region } & 127\end{array}$

$\begin{array}{ll}\text { Appendix B } & 131\end{array}$

B.1 Scaling of the Velocity Field . . . . . . . . . . . . . . 131

B.2 Scaling of the Coordinate System . . . . . . . . . . . . . 134

B.3 Scaling an Obstacle . . . . . . . . . . . . . . . . 134

B.4 Effect of $V_{\infty} \ldots \ldots \ldots \ldots \ldots \ldots \ldots$

$\begin{array}{ll}\text { Appendix C Definitions } & 137\end{array}$ 


\section{List of Tables}

2.1 Comparison of TP and ROA characteristics . . . . . . . . . . 12

3.1 Computing time for different number of panels . . . . . . . . . . . . 49

4.1 Aerosonde UAV specifications. . . . . . . . . . . . . . . . . 68

A.1 Different wedge length required for a specific scenario based on the choice of $\delta \ldots \ldots \ldots \ldots \ldots \ldots \ldots \ldots$ 


\section{List of Figures}

1.1 GeoSurv II prototype $\ldots \ldots \ldots \ldots \ldots \ldots \ldots$

1.2 Example of a survey path (shown with white lines) $\ldots \ldots \ldots .4$

1.3 Snapshots from a flight during a geophysical flight survey (Courtesy of

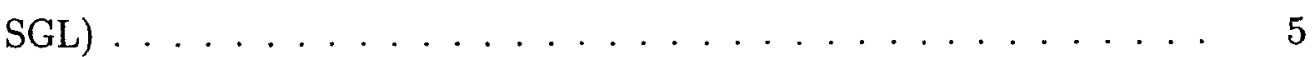

1.4 Left and right stereo image pair (balloon obstacle circled in red) $\ldots 6$

1.5 Stereoscopic image computed for the right input image (lighter regions are closer to the camera $\ldots \ldots \ldots \ldots \ldots \ldots$

1.6 Connected component segmentation results with similar depth regions.

Obstacles are highlighted in red bounding boxes. . . . . . . . 8

2.1 Visibility graph $\ldots \ldots \ldots \ldots \ldots \ldots \ldots \ldots \ldots$

2.2 Flight primitives $\ldots \ldots \ldots \ldots \ldots \ldots \ldots \ldots \ldots \ldots$

2.3 The RRT method used for path planning in an urban area . . . . 15

3.1 APF and artificial forces corresponding to a circle obstacle and a goal 24

3.2 The value of four potential functions $\ldots \ldots \ldots \ldots \ldots$

3.3 The value of four harmonic functions $\ldots \ldots \ldots \ldots \ldots$

3.4 Obstacle avoidance using potential flow . . . . . . . . . 30

3.5 Uniform steady-state flow $\ldots \ldots \ldots \ldots \ldots$

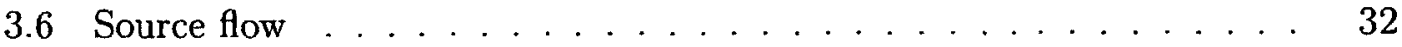

3.7 Volumetric flow from a source $\ldots \ldots \ldots \ldots \ldots \ldots \ldots$

3.8 Source sheet . . . . . . . . . . . . . . . . . . 35 
3.9 An n-panel obstacle . . . . . . . . . . . . . . . . 36

3.10 Computing the contribution of the $i^{t h}$ panel to $V_{n_{i}} \ldots \ldots \ldots$

3.11 Velocity field and flow pathlines around a unit square. $V_{\infty}=1 \mathrm{~m} / \mathrm{s} . .42$

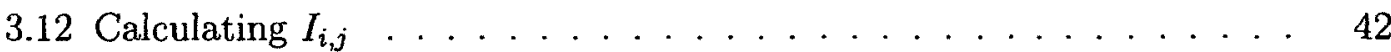

3.13 Assuming equal depth and width in obstacle modeling $\ldots . . . .45$

3.14 Difference between exact contour and assumption based on equal width and depth . . . . . . . . . . . . . . . . . 46

3.15 Obstacle being updated $\ldots \ldots \ldots \ldots 46$

3.16 Avoiding pathlines for a random polygon . . . . . . . . . . . 47

3.17 Concave obstacle ... . . . . . . . . . . . . . . 50

3.18 Concave obstacle (concave region is magnified) . . . . . . . . 50

3.19 Concave region of a concave obstacle with enhanced accuracy . . . . 51

3.20 Top view of an aircraft performing the tightest turn possible . . . . . 51

3.21 Front view of an aircraft performing the tightest turn possible ( $\phi$ is the

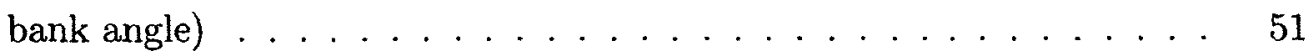

3.22 Side view of an aircraft performing the tightest pull-up turn possible . 52

3.23 Simple unit length square . . . . . . . . . . . . . . . 54

3.24 Square with wedges added . . . . . . . . . . . . 54

3.25 Modelled obstacle . . . . . . . . . . . . . . . . . . . . 54

3.26 Computing approximate radius of curvature using two adjacent points 55

3.27 Three pathlines in close proximity of the horizontal axis of symmetry 56

3.28 Minimum radius of curvature for different wedge lengths. Pathlines with starting points within $[-0.1,0.1] a$ of the centerline were excluded. 57

3.29 Increasing obstacle size to avoid pathlines with tight turns . . . . 58

3.30 Enumeration of panels for multiple obstacles $\ldots \ldots \ldots$

3.31 Two obstacles of unit length with wedges $h=0.92(a=1) \ldots 59$ 
3.32 Velocity field flow pathlines around a unit square. $V_{\infty}=1 \mathrm{~m} / \mathrm{s}, \Lambda_{g}=$

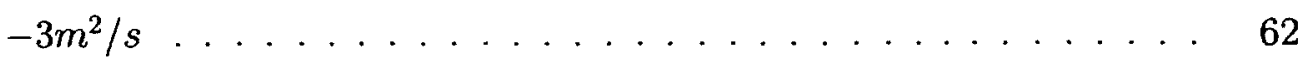

3.33 A uniform flow and a sink. $V_{\infty}=1 \mathrm{~m} / \mathrm{s}, l=2 m$ and $\Lambda=-2 \mathrm{~m}^{2} / \mathrm{s} \ldots \quad 64$

4.1 The Aerosonde UAV model at the museum of flight in Seattle . . . 66

4.2 Aerosonde Simulink model and FlightGear visualization for a $0.05 \mathrm{rad}$ aileron deflection ..................... 67

4.3 Autonomous flight architecture . . . . . . . . . . . . 68

4.4 A mission consisting of FlyTo and From To commands . . . . . . . . . 69

4.5 Autopilot architecture . . . . . . . . . . . . . . . 70

4.6 Heading from crosstrack error controller . . . . . . . . . . . . 71

4.7 Definition of crosstrack error that is used in a FromTo controller . . . 71

4.8 Autonomous flight architecture with OA . . . . . . . 73

4.9 Obstacle avoidance hierarchy . . . . . . . . . . . . 74

4.10 Autopilot architecture for OA . . . . . . . . . . 76

$4.11+90$ degree heading command . . . . . . . . . . . 77

$4.12+90$ degree course command . . . . . . . . . . . . . 77

4.1320 degree pitch command . . . . . . . . . . . 78

4.1420 degree path angle command ... . . . . . . . . 78

$4.15+90$ degree flight scenario . . . . . . . . . . . . . 78

$4.16+90$ degree heading command. Wind $=5 m / s \ldots \ldots . \ldots 79$

$4.17+90$ degree course command. Wind $=5 \mathrm{~m} / \mathrm{s} \ldots \ldots \ldots 79$

$4.18 \mathrm{Crab}$ angle while flying in wind . . . . . . . . . . 80

4.1920 degree pitch command with $2 \mathrm{~m} / \mathrm{s}$ downdraft $\ldots \ldots$. . . . . 80

4.2020 degree path angle command with $2 \mathrm{~m} / \mathrm{s}$ downdraft . . . . . . . . 80

5.1 Three-dimensional view of an aircraft encountering an obstacle scenario 82

5.2 Top plane obstacle scenario ................. 83

5.3 Side plane obstacle scenario . . . . . . . . . . . . . 83

xiii 
5.4 Obstacle Avoidance Range (Top view) . . . . . . . . . . . . 84

5.5 Correlation between $y_{\max }$ and wedge length, $h \ldots \ldots 85$

5.6 Combining explicit and implicit obstacles (top plane) ... . . . 85

5.7 Simultaneously considering an explicit and implicit obstacle . . . 86

5.8 Simultaneously considering two explicit obstacles $\ldots \ldots . . \ldots 87$

5.9 Artificially magnifying obstacles in the side plane $\ldots \ldots . \ldots 88$

5.10 Combining two explicit obstacles (side plane) . . . . . . . . . . . . 89

5.11 Resizing obstacles in the side plane to guarantee flying over the obstacle 89

5.12 Projection of avoiding maneuver on obstacle . . . . . . . . . 91

5.13 Activation Area (AcA) in the top plane . . . . . . . . . . . 92

5.14 Maximum course/path angle $(0<h / a<2$ with 0.1 increments $)$. . . 93

5.15 Defining $l$, to compute $\Lambda_{g} \ldots \ldots \ldots \ldots$

5.16 The returning effect caused by a sink . . . . . . . . . . 96

5.17 Fly-around maneuver for an obstacle of length $50 \mathrm{~m}$, where $\delta_{y}=20 \mathrm{~m} \quad 98$

5.18 Fly-around maneuver for an obstacle of length $50 \mathrm{~m}$, where $\delta_{y}=10 \mathrm{~m} \quad 99$

5.19 Fly-around maneuver for an obstacle of length $50 m$, where $\delta_{y}=0 m \quad . \quad 99$

5.20 Avoidance maneuver with updated obstacle depth while performing the avoidance maneuver $\ldots \ldots \ldots 100$

5.21 Fly-over maneuver for an obstacle of $75 \mathrm{~m}$ height, where $\delta_{z}=15 \mathrm{~m} \ldots 101$

5.22 Fly-over maneuver for an obstacle of $75 \mathrm{~m}$ height, where $\delta_{z}=7.5 \mathrm{~m} .101$

5.23 Fly-over maneuver for an obstacle of $75 m$ height, where $\delta_{z}=0 m$. . 102

5.24 Fly-over maneuver for an obstacle of $75 m$ height, where $\delta_{z}=37.5 m \quad \cdot 102$

5.25 Two obstacle scenario - Obstacle dimensioning . . . . . . . . . . . 104

5.26 Two obstacle scenario - Flight case I . . . . . . . . . . . . 104

5.27 Two obstacle scenario - Flight Case II . . . . . . . . . . . . . . 105

5.28 Two obstacle scenario - Flight case III . . . . . . . . . . . 106

5.29 OA maneuvers in a scenario with two obstacles for three different cases. 107 
6.1 The Senior Telemaster . . . . . . . . . . . . . . . . . 110

6.2 The transmitter (left) and receiver (right) . . . . . . . . . . 111

6.3 The MP2028 autopilot . . . . . . . . . . . . . . . . . 112

6.4 Horizon GCS showing ATB 10 in flight . . . . . . . . . . . 113

6.5 MicroHard ${ }^{\circledR}$ n920-ENC radio modem . . . . . . . . . . . . . . . 113

6.6 ATB 10 and ground control station setup . . . . . . . . . . . 114

6.7 Fine-tuning controller gains over 10 laps (laps were flown clockwise) . 115

6.8 Circuit flown with fine-tuned autopilot (lap was flown clockwise) . . 115

6.9 Survey flown with fine-tuned autopilot under strong wind with violent gusts ............................ 116

6.10 ATB 10 during autonomous flight $\ldots \ldots \ldots . \ldots . \ldots 117$

6.11 Obstacle avoidance test setup . . . . . . . . . . . 118

A.1 Minimum radius of curvature for different wedge lengths for $\delta=0.02 \quad 127$

A.2 Minimum radius of curvature for different wedge lengths for $\delta=0.04128$

A.3 Minimum radius of curvature for different wedge lengths for $\delta=0.06 \quad 128$

A.4 Minimum radius of curvature for different wedge lengths for $\delta=0.08 \quad 129$

A.5 Minimum radius of curvature for different wedge lengths for $\delta=0.1 \quad 129$

C.1 Aircraft course and heading . . . . . . . . . . . . . . 137

C.2 Aircraft path angle . . . . . . . . . . . . . . . 138 


\section{List of Acronyms}

\begin{tabular}{|c|c|}
\hline Acronyms and Abbreviations & Definition \\
\hline AcA & Activation Area \\
\hline AGL & Above Ground Level \\
\hline $\mathrm{AO}$ & Autonomous Operations \\
\hline APF & Artificial Potential Field \\
\hline$A R$ & Avoidance Range \\
\hline ATB & Avionics Test Bed \\
\hline $\mathrm{CIC}$ & Computer In Control \\
\hline $\mathrm{CO}$ & Considered Obstacle \\
\hline $\mathrm{ESC}$ & Electronic Speed Controller \\
\hline FHSS & Frequency-hopping spread spectrum \\
\hline GCS & Ground Control Software \\
\hline GPS & Global Positioning System \\
\hline $\mathrm{Li}-\mathrm{PO}$ & Lithium Polymer \\
\hline
\end{tabular}


LLA

$\mathrm{MO}$

MUAV

NSERC

OA

OD

PIC

PID

$\mathrm{RC}$

ROA

RX

SISO

SGL

$\mathrm{TP}$

TX

UAV

WP
Latitude, Longitude and Altitute

Modelled Obstacle

Micro-Unmanned Aerial Vehicle

Natural Sciences and Engineering

Research Council of Canada

Obstacle Avoidance

Obstacle Detection

Pilot In Command

ProportionalIntegralDerivative

Radio Control

Reactive Obstacle Avoidance

Receiver

Single-Input Single-Output

Sander Geophysics Limited

Trajectory Planning

Transmitter

Unmanned Aerial Vehicle

Waypoint 


\section{Chapter 1}

\section{Introduction}

Unmanned Aerial Vehicles (UAVs) are being developed in a variety of applications, mainly for military purposes. However, research and demand for civilian applications are increasing. Some examples of such applications are: geophysical surveys [1], forest fire monitoring [2], environmental monitoring [3], border patrol [4], aerial photography [5], monitoring pipelines [6] and even wildlife research [7].

The use of UAVs can be attractive for several reasons. Firstly, UAVs can operate in situations too dangerous for human pilots. Secondly, UAVs can be flown at lower altitudes than manned aircraft [8]. For example, this feature is attractive for geophysical surveys where lower flight increases the resolution of measured data. Finally, due to the absence of an on-board pilot, smaller aircraft can typically be used, which decreases the price of the aircraft and yield to savings in fuel consumption.

UAVs have different levels of autonomy. In the absence of autonomy a UAV can only be remotely piloted. At a basic level of autonomy, a flight trajectory is preplanned and the aircraft has the ability to complete a mission. If the system has the ability to avoid obstacles and potential collision scenes, the autonomy level of the aircraft is even higher. In such a UAV, the operator uploads a flight mission to the UAV, and only monitors the flight. However, the level of autonomy of a UAV is not limited to what is mentioned here. For instance, in forest fire monitoring, a UAV can 
be given the ability to define its flight trajectory while flying, in a way to follow the boundaries of the forest fire [2].

\subsection{GeoSurv II Project}

The GeoSurv II project is funded jointly by Sander Geophysics Limited (SGL) and NSERC. Its objective is to develop a small UAV, capable of performing high resolution geophysical surveys at low flight altitudes. To date, the aircraft has been designed, built, and several low and high-speed taxi tests have been performed. The aircraft designed is a twin-boom pusher aircraft with a wingspan of $4.9 m$, with two magnetometers installed on its wing tips to measure the magnetic field of the terrain the UAV is flying over. The GeoSurv II prototype is illustrated in Figure 1.1.

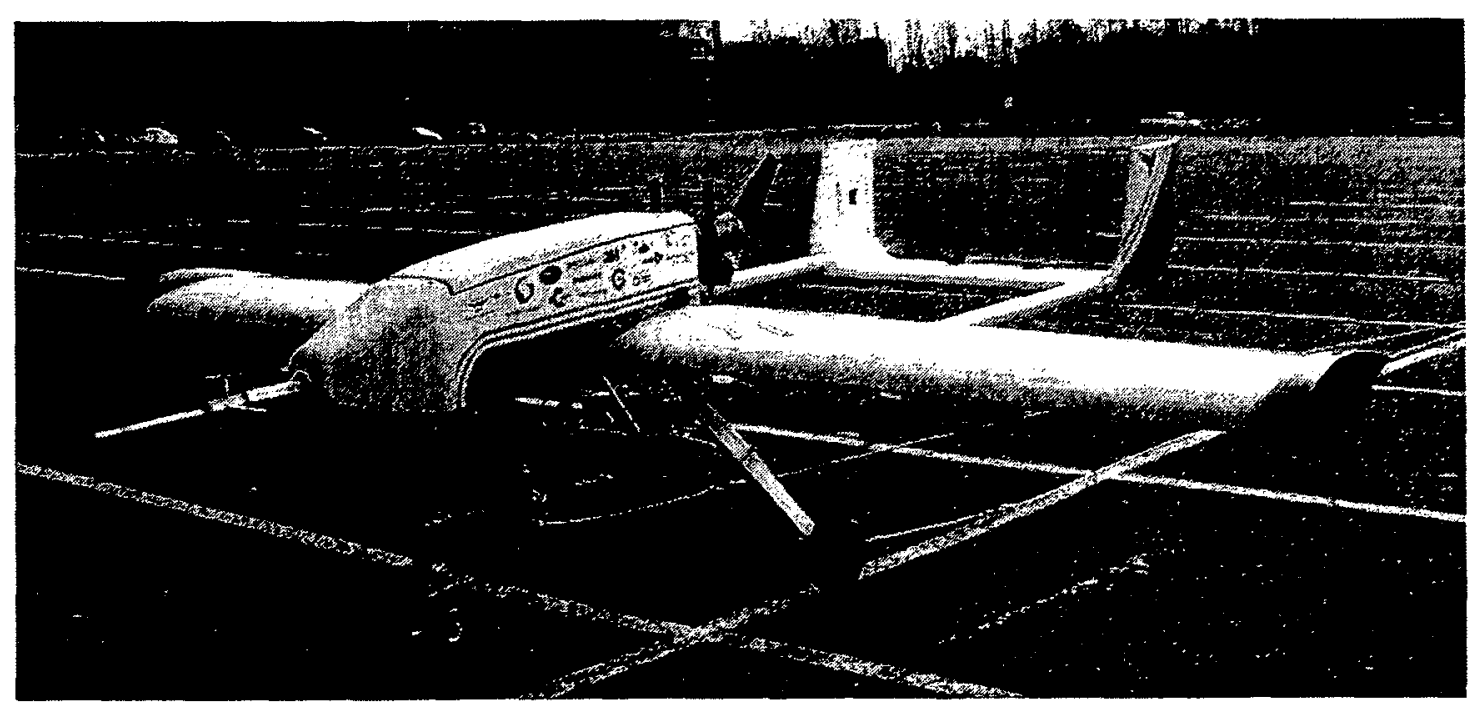

Figure 1.1: GeoSurv II prototype

Since the aircraft's mission is to measure magnetic field, research has been undertaken on reducing magnetic interference of ferromagnetic sources on the aircraft. Furthermore, research is being carried-out on developing a low cost composite structure for the airframe. 
Currently, to perform surveys similar to those expected from GeoSurv II, a four member crew is required. The crew consists of a pilot and a co-pilot, an aircraft maintenance engineer and a geophysicist. One of the objectives of the GeoSurv II project is to develop a UAV with a level of autonomy where the maintenance engineer and the geophysicist would be able to perform the survey without the pilots.

Therefore, another area of research within the GeoSurv II project is Autonomous Operations $(A O)$. The research within this area is aimed to develop a system that has a level of autonomy as stated above. Such a system should have a "sense and avoid" feature to automatically avoid obstacles without the interference of the operator. The sense and avoid feature is broken into two separate research areas: Obstacle Detection $(O D)$ and Obstacle Avoidance (OA). In this thesis OA will be covered.

\subsubsection{Survey Path}

The geophysical survey consists of multiple survey lines that are equally spaced over the area of interest. In order to account for terrain, a digital elevation model (DEM) of the survey area is used. This model is provided by the Shuttle Radar Topography Mission (SRTM) and is accessible through The Consultative Group on International Agricultural Research (CGIAR). The survey lines are then offset from the DEM in order to keep the aircraft at a constant height AGL (Above Ground Level) [9]. For the measured magnetic fields to have a high-resolution the aircraft is desired to fly below $50 \mathrm{~m}$ AGL. In Figure 1.2 the survey lines are shown for a test survey [9].

\subsubsection{Potential Obstacles}

With flight altitudes below $50 \mathrm{~m}$ AGL, the main obstacles the UAV may encounter are powerline and powerline towers, telecommunication towers and trees. Although the UAV is not intended to fly over urban areas; buildings, silos and industrial chimneys 


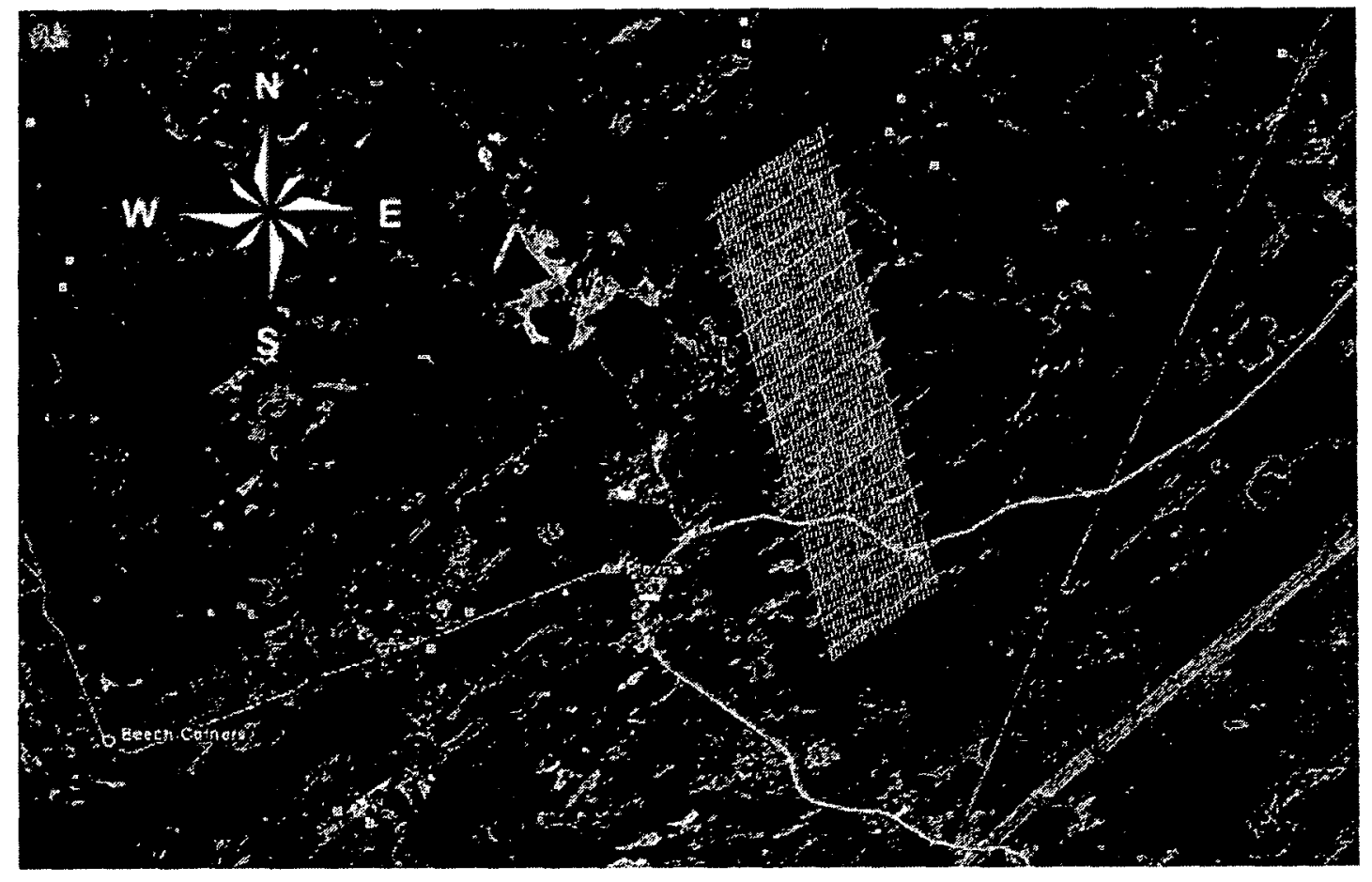

Figure 1.2: Example of a survey path (shown with white lines) 
are also potential obstacles that may not be known prior to flight. In Figure 1.3, several snapshots are extracted from a video taken while performing tests for a geophysical survey. These pictures give an insight into the type of terrain GeoSurv II will be flown over.

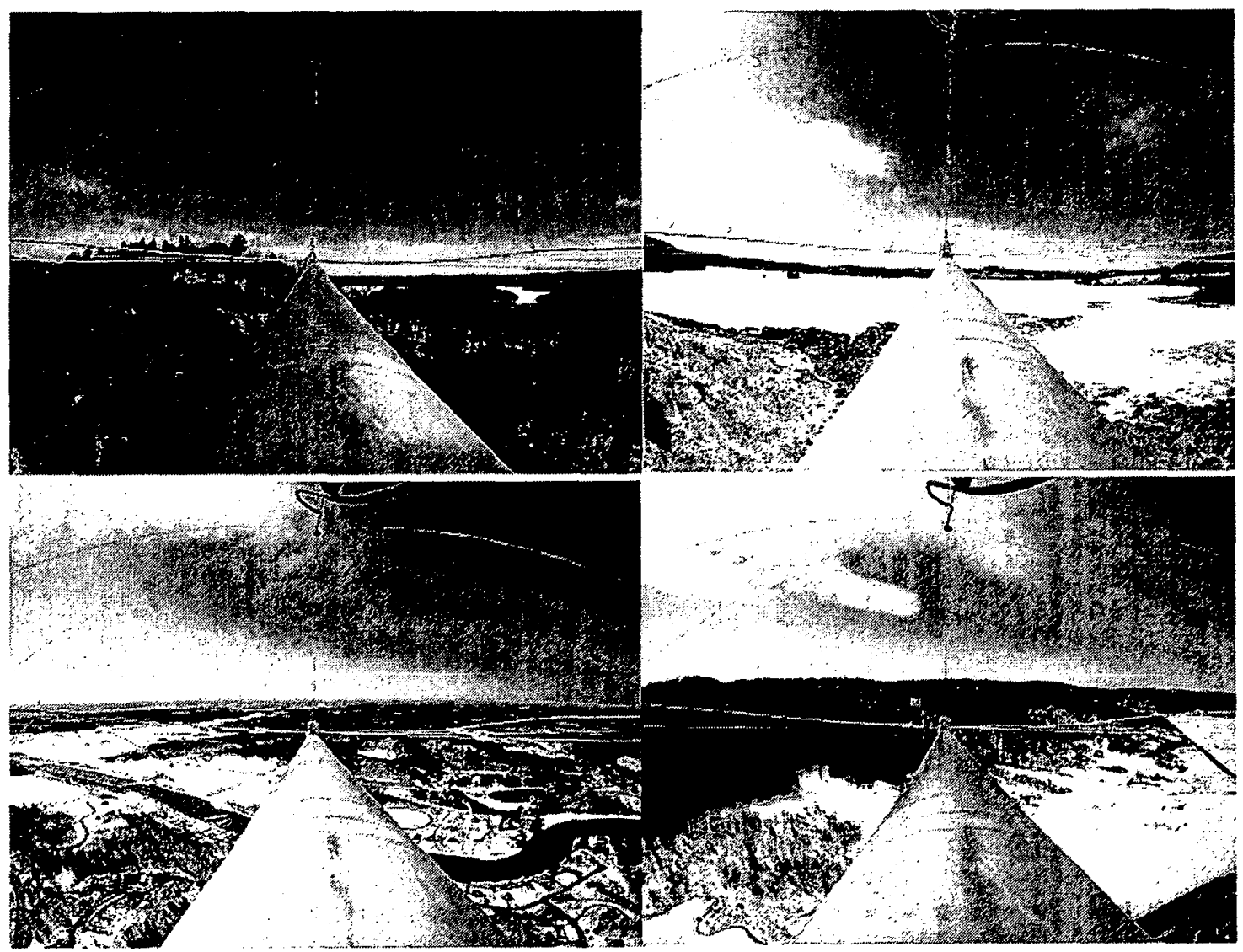

Figure 1.3: Snapshots from a flight during a geophysical flight survey (Courtesy of SGL)

At this point the aircraft is not expected to fly in non-segregated airspace. Therefore other aircraft are not considered as potential obstacles. Finally, it should be noted that the UAV is not intended to perform in cluttered space such as in an urban area where the UAV has to fly between a large number of buildings. 


\subsubsection{Obstacle Detection}

In order to be able to avoid obstacles, they first need to be detected. The obstacle detection being developed for the GeoSurv II currently focuses on using stereoscopic vision to determine the depth to visual features in front of the aircraft The obstacle detection method presented here is a summary of the work performed in Ref. [10], and further details may be found there.

Firstly, a pair of images is taken from cameras mounted on each wing of the aircraft. In Figure 1.4, such a pair of images is shown. Note that in this setup, a group of balloons were set in the field to be detected.

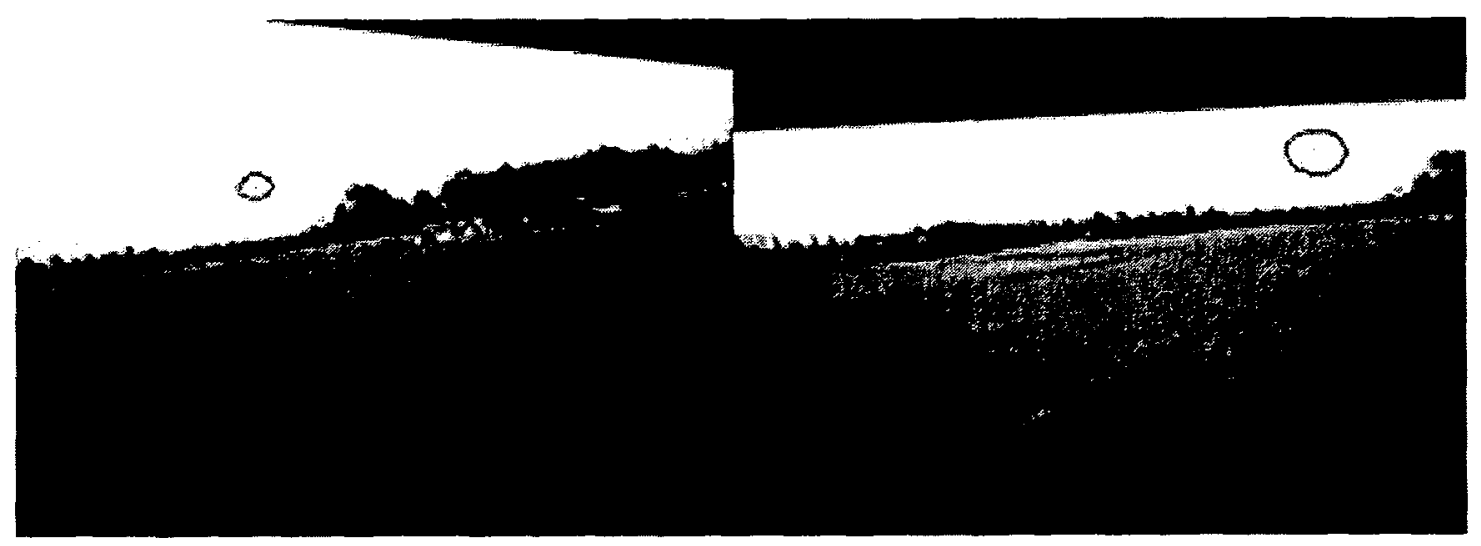

Figure 1.4: Left and right stereo image pair (balloon obstacle circled in red)

Secondly, edge detection is applied to the images to look for dominant line features. The edge map is then thickened and is used to mask the original color images. The result of this processing is the feature extraction of any potential edges which could belong to a potential obstacle. Then, stereoscopic block matching is applied to the detected edges so.that their corresponding depth can be detected. The result is shown in Figure 1.5, with lighter regions representing distances closer to the camera.

Finally, a connected components algorithm is applied in order to group regions of similar depth. This is shown in Figure 1.6 with red rectangles enclosing each individual region. Note how the balloons are now clearly identifiable as obstacles in 


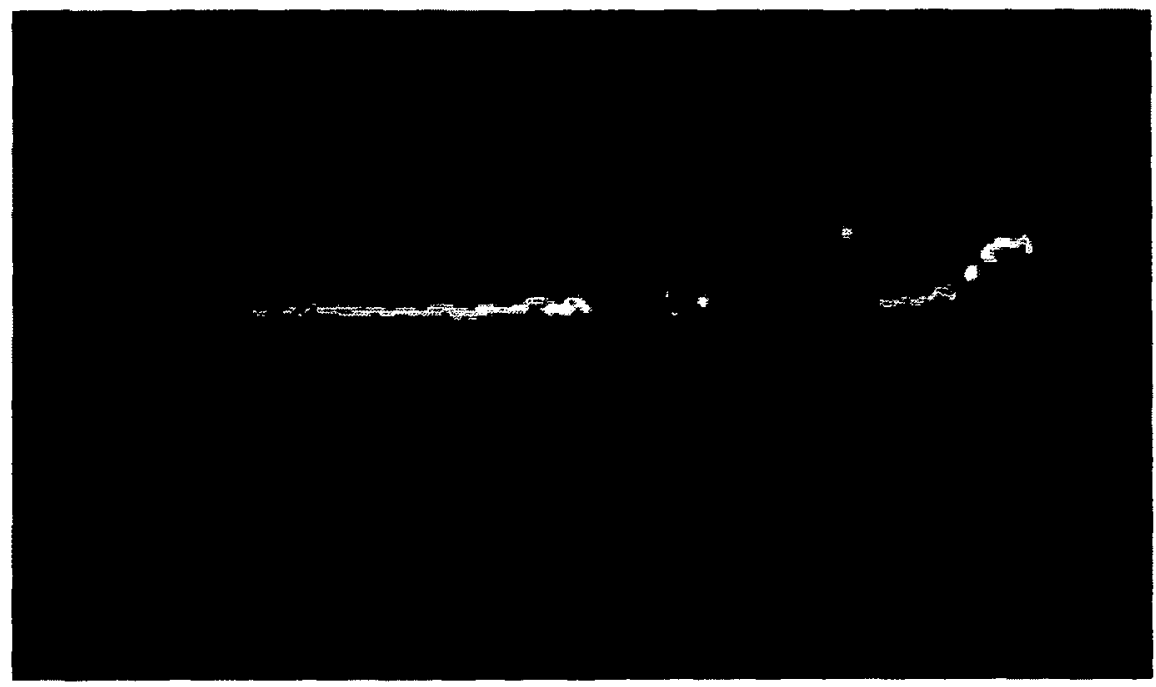

Figure 1.5: Stereoscopic image computed for the right input image (lighter regions are closer to the camera)

the figure. A depth value is associated to each rectangle and the locations and sizes of all bounding rectangles are then passed to OA.

\subsection{Thesis}

\subsubsection{Thesis Motivation}

The GeoSurv II UAV is expected to have a high level of autonomy. The operator of the UAV should only have to define a flight survey for the UAV (Section 1.1.1) and the UAV should be able to perform the flight with minimal interference of the operator. This level of autonomy requires the UAV to be able to avoid obstacles that were not foreseen and that are detected while flying. The motivation of this thesis is to develop a method to avoid these obstacles. In Chapter 2, it will be seen that this type of avoidance is termed as ROA. 


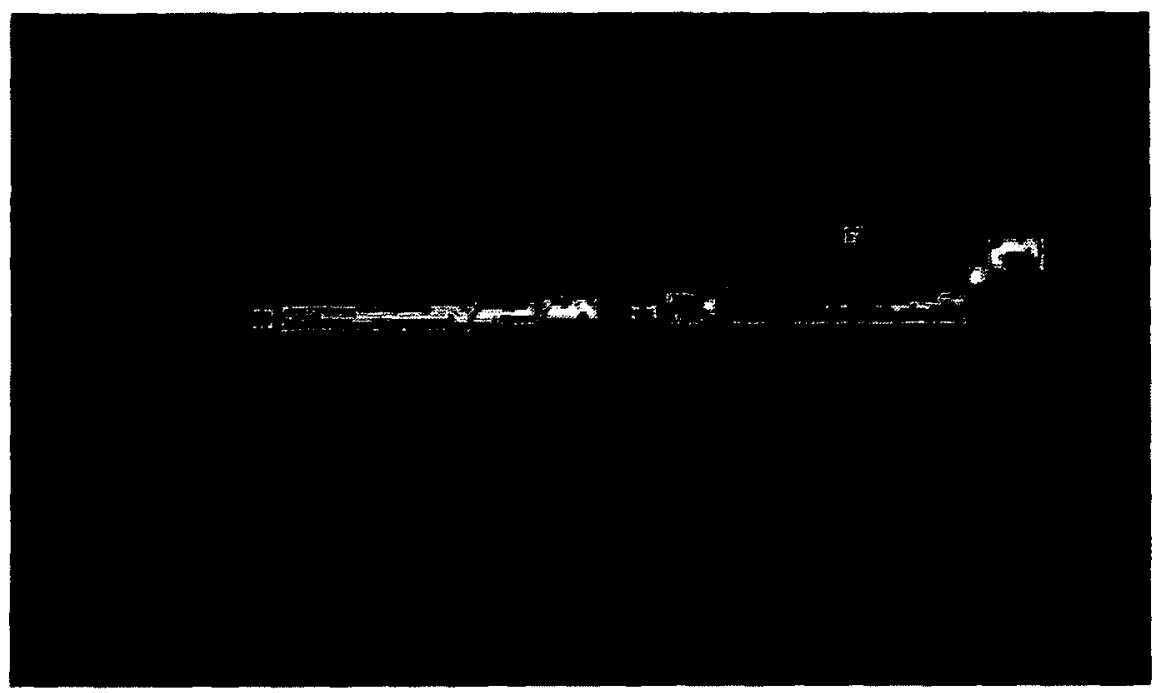

Figure 1.6: Connected component segmentation results with similar depth regions. Obstacles are highlighted in red bounding boxes.

\subsubsection{Problem Statement and Thesis Structure}

The objective of this thesis is to develop a method of obstacle avoidance that is achievable in real-time and with limited computation resources that can be installed on an aircraft. The developed method is to consider fixed-wing aircraft constraints such as minimum turning and pull-up radii, so that it does not command maneuvers to the aircraft that it cannot perform. Moreover, the method is designed to cope with potential obstacles that exist in low level flight.

In Chapter 2, a review of the literature for obstacle avoidance is performed and analysis is done to choose a method. In Chapter 3, the theory of the chosen method, i.e. potential flow, is described in detail. In Chapter 4, the autonomous flight architecture used in this thesis is described and the simulation framework that obstacle avoidance is tested on is introduced. In Chapter 5, the implementation of OA in the autonomous flight architecture is demonstrated. Moreover, the results of OA using potential flow are shown on the simulation framework and the results are discussed. In Chapter 6, a test bed developed within the framework of this thesis for autonomous 
operations is introduced and initial results of autonomous flight are demonstrated. Finally in Chapter 7, the conclusions and research contributions are summarized. 


\section{Chapter 2}

\section{Background Information and Method Selection}

Obstacle avoidance $(\mathrm{OA})$ is a topic that has been researched in different fields that require autonomous operation. The first field where it was researched was robotics [11-15]. With the advent of UAVs, and the increasing desire for autonomous UAVs, robotic obstacle avoidance methods were employed for UAVs [16-18]. However, because UAVs and robots have different properties and constraints, the methods being used on UAVs had to be specifically tailored to their new application. For instance, fixed-wing UAVs cannot stop in flight and change direction, whereas robots and rotary-wing UAVs can afford such luxury. Another example is that fixed-wing UAVs have a minimum turning and pull-up radius that need to be taken into account when designing OA methods. In summary, UAVs and robots can be divided into two categories: rotary-wing UAVs and mobile robots, which have high maneuverability, and fixed-wing UAVs and robotic manipulators which have more limited maneuverability.

In the literature, OA has been performed under two different set of circumstances: (1) When obstacle positions and properties are known prior to the start of a mission, the avoiding method is known as Trajectory Planning (TP). Other terminologies such as, path planning, global guidance and global path planning have been used as well. 
(2) In contrast, when the existence of obstacles are unknown and they "pop-up" in real-time, the method is called Reactive Obstacle Avoidance (ROA) or local path planning.

The avoidance at the global and local level (TP and ROA), operate under different circumstances. Thus, the methods used to perform avoidance have different characteristics. In TP, the method's response time is not of particular concern because the algorithms are run before the mission starts with no concern of algorithm response time. Similarly, the amount of computation power and memory required is not a limitation, since the algorithms can be run on a powerful PC. In contrast, response time in ROA is of great importance because delay in evasive commands can lead to collision.

Memory resources and computation power may also be limited on board an aircraft. This limitation can become more restrictive when working with MicroUnmanned Aerial Vehicles (MUAVs).

Furthermore, in ROA the robustness of the method employed is of great concern, since there is very limited time to detect and correct errors (due to the speed of the UAV relative to an obstacle), which can potentially lead to collision. In contrast, in $\mathrm{TP}$, the generated path can always be verified for collision before being confirmed for a mission and if needed trial and error can be used.

Finally, in TP, as there is no time constraint on the computation of the trajectory, the optimality of the generated path is typically a design parameter of interest. In contrast, in ROA, optimality is not crucial and compared to robustness has a lower degree of importance. Table 2.1 summarizes the importance of the characteristics discussed above for TP and ROA.

Furthermore, if a UAV would be required to fly in a non-segregated airspace, it should have the ability to avoid other UAVs/aircraft. In the literature, the research carried out to develop methods for this purpose is called collision avoidance [19-22]. 


\begin{tabular}{|l||c|c|}
\hline Characteristics & TP & ROA \\
\hline \hline Response Time & Unimportant & Very low \\
\hline Computation resources & Unlimited & Limited \\
\hline Robustness & Advantageous & Crucial \\
\hline Optimality & Important & Advantageous \\
\hline
\end{tabular}

Table 2.1: Comparison of TP and ROA characteristics

\subsection{Literature Review}

In this section, the previously presented OA methods are discussed in further detail. Many of these methods were developed for robotic manipulators and some of them were later applied to UAVs. The strengths and weaknesses of these methods are discussed and the possibility of employing them for fixed-wing UAVs is assessed. Note that since the UAV is not expected to fly in non-segregated airspace in this research, collision avoidance methods are not of interest.

\subsubsection{Road Map Method}

A road map is a network of straight lines that do not intersect obstacles and connect the starting point to the goal of a robot. To create such a network, the operational space of the robot is divided into an obstacle-free subspace and an obstacle subspace. Then, a network of straight lines within the obstacle-free subspace is created. Using a graph search algorithm, a path is then chosen within that network, which is guaranteed to be obstacle free [23]. Different algorithms such as, Dijkstra and A* have been employed to perform the graph search; some being faster at the price of losing optimality [24].

Visibility graphs [23] are the simplest of road maps. Firstly, it is considered that all obstacles are polygons -or are fitted with polygons. Considering the vertices of these polygons, the starting point and the goal point, any two vertices that are within 
line-of-sight are connected. As a result, a network is created that never crosses the obstacles (Figure 2.1).

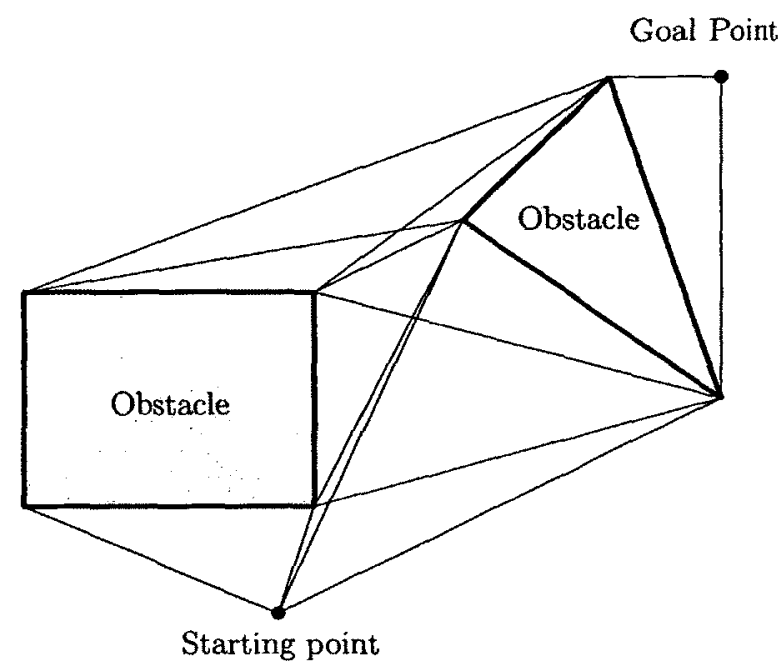

Figure 2.1: Visibility graph

Voronoi diagrams [23] have also been used to create road maps. The Voronoi diagrams result in road maps by creating fences around obstacles. The fences are created by drawing lines perpendicular to the lines connecting the centers of obstacles. Then, the fences are adjusted to meet a minimum number of vertices.

The road map method is not computationally expensive to generate. However, the trajectories created do not directly take kinematic or dynamic constraints into account. In Ref. [25], Voronoi diagrams were used to generate a path for a UAV with minimal exposure to a priori known radars. In this approach, the Voronoi diagrams were fitted with arcs at the diagram's vertices to incorporate the aircraft's minimum turning radius. Nevertheless, in a cluttered environment, the arcs might cross obstacles, and therefore trial and error is required.

In Ref. [16], a two-level architecture for UAV OA was proposed that consisted of TP and ROA. For TP, a road map was created by a node expansion technique while considering aircraft kinematic constraints. For ROA, a trajectory was created by 
interconnecting a set of dynamically feasible primitives. These primitives were short maneuvers that were feasible for the aircraft and were a function of the aircraft's starting state. In Figure 2.2 [16], the feasible flight primitives are shown for a specific starting point. A graph search method was then used to connect a number of feasible

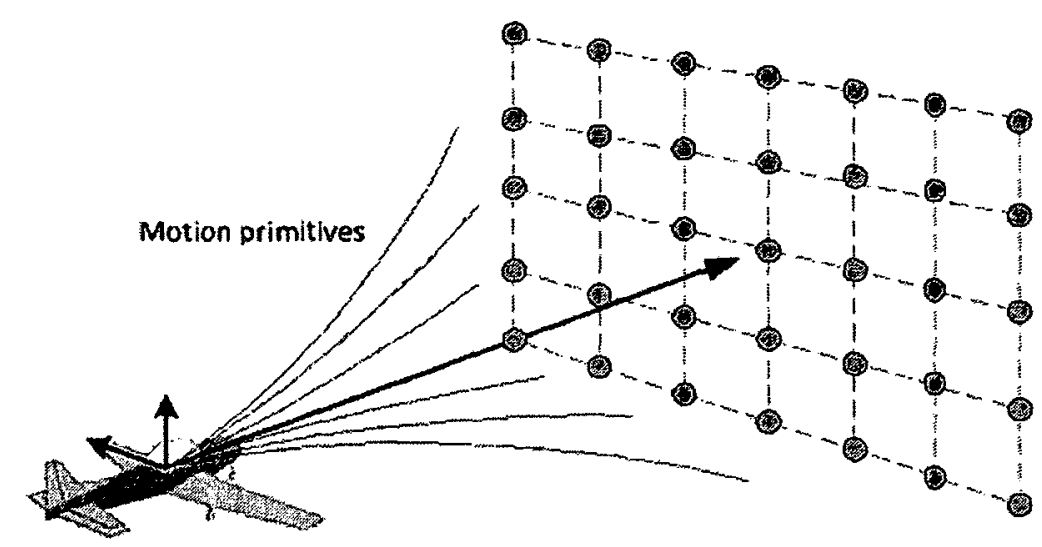

Figure 2.2: Flight primitives

primitives that would avoid the obstacle. A shortcoming of this method is that for different aircraft, a complex set of primitives need to be estimated. In addition, the storage of these motion primitives requires a large memory [26]. In summary, the road map method is more suited to TP than ROA.

\subsubsection{Rapidly Exploring Random Tree}

Rapidly Exploring Random Tree [27,28], RRT, is a probabilistic method, to find an obstacle free path in a cluttered environment. Firstly, the starting point of the path is used to initiate a "tree". Secondly, a random point is generated and the nearest node on the tree to this random point is found. Thirdly, a new node is created on the line connecting the random point and the nearest node from the previous step; 
at an incremental distance from the nearest node. Finally, if this point does not collide with any obstacle it is added to the tree. This process is done in an iterative manner until the goal is reached. Note that in a cluttered environment, this collision verification is computationally intensive [26]. In Ref. [17], Amin et al. applied the constraints of a rotary-wing aircraft to the RRT method. To achieve this, an extra verification step was added to the RRT algorithm: at each step while expanding the tree, the new segments were checked to satisfy a maximum turn rate. The results were trajectories that could be commanded to the UAV. Unfortunately, they were not tested on the UAV or in simulation. Therefore the method's ability to run in realtime was not assessed. Similarly, in Ref. [18], the RRT method was implemented on a flying wing MAV, where the the algorithm took up to five seconds to find an obstacle free trajectory through an urban area. The growth of the tree is shown in Figure $2.3[29]$.

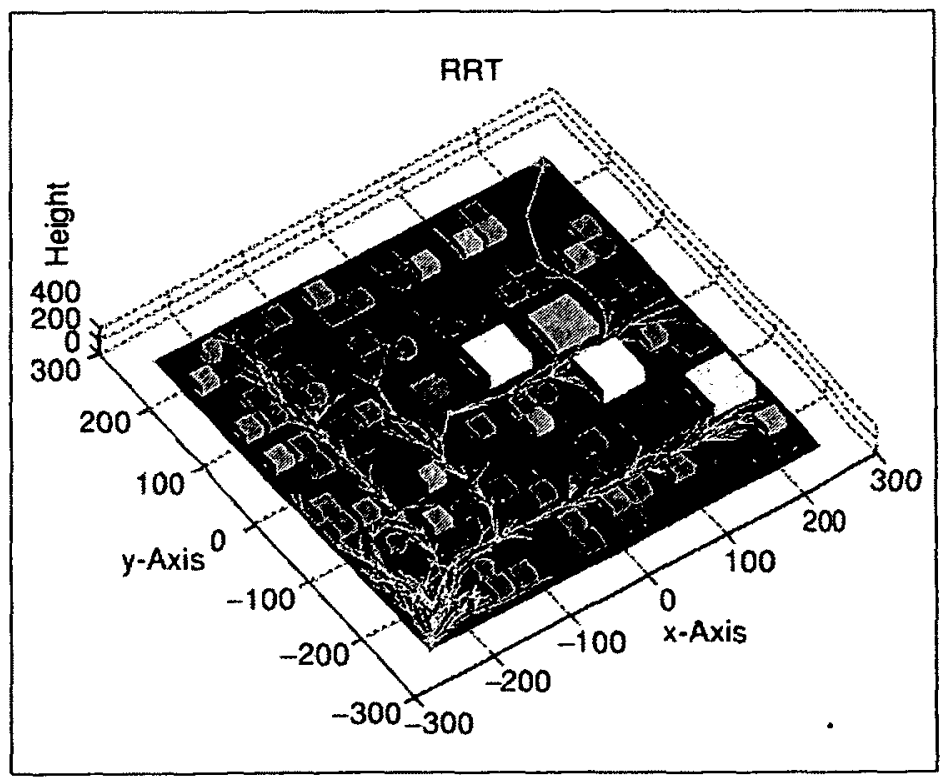

Figure 2.3: The RRT method used for path planning in an urban area

In summary, the method is computationally intensive and although it is a strong 
candidate for TP in a heavily cluttered environment, it is not suitable for ROA.

\subsubsection{Artificial Potential Fields}

The first initiative for ROA was taken for robotic manipulators in [15]. In this initiative, a repulsive Artificial Potential Field (APF) was assigned to an obstacle present in the operational space of a robotic manipulator. An attractive APF was also assigned to the goal the manipulator had to reach. As a result, the APFs generated an attractive force (implemented by actuators), which constantly pulled the manipulator's end-effector toward the goal, while in the presence of an obstacle, a repulsive force was generated (also implemented by the actuators) to avoid collision. In Ref. [15], Khatib stated that the APF method could be extended to multiple obstacles and to moving obstacles.

Khatib's repulsive potential field was defined as

$$
\Phi_{o}(\vec{x})= \begin{cases}\frac{1}{2} \eta\left(\frac{1}{f(\vec{x})}-\frac{1}{f(\vec{x})}\right)^{2} & f(x) \leqslant f\left(x_{0}\right) \\ 0 & f(x)>f\left(x_{0}\right)\end{cases}
$$

where $f(x)=0$ defines the surface of the obstacle and $x_{0}$ is a given point in the vicinity of the obstacle. Furthermore, the artificial force was defined as

$$
\vec{F}_{o}=-\nabla\left(\Phi_{o}(\vec{x})\right)
$$

A virtue of the APF method is its high computation speed. This simplicity is a result of an analytic derivation of the artificial forces. Another virtue of APFs, is that the resultant guiding force can be assumed to be exerted to the manipulator's endeffector and this force can be incorporated in the joint torque commands needed to perform an evasive maneuver. However, this last feature is only beneficial for robotic 
manipulators.

The major weakness the APF method has, is the occurrence of local minima in the overall potential field of an obstacle and a goal [30]. A local minimum results in zero force being exerted to the robot. This zero force leaves the end-effector stranded where the local minimum occurs.

In Ref. [30], the potential field was modified to a superquadratic potential field. The modified function was tested on a robotic manipulator and a single obstacle was successfully avoided and the goal was reached. In the same scenario, the end-effector was stranded while using Khatib's potential function. Furthermore, the robotic manipulator was able to successfully avoid a scenario with four obstacles. However, this improvement in the APF method did not guarantee immunity against local minima in the presence of multiple obstacles.

In the previous developments of the APF method, the goal was always assumed to be far from obstacles. However, if the goal is close to an obstacle, the repulsive force generated by the obstacle can be larger than the attractive force of the goal and as a result the robot will miss the goal point. In Ref. [31], the APF was modified to solve this problem by taking the distance between the robot and the goal into account when defining the APFs. In Ref. [32], a new APF was proposed that could be used for moving obstacles and goals. Note that in [31] and [32], the problem of local minima was not addressed.

\subsubsection{Harmonic Potential Functions}

To circumvent the limitation of the APF.method in the scenario of multiple obstacles, the usage of harmonic potential functions was suggested in Ref. [33]. It was proven that the addition of any number of harmonic potential functions (corresponding to multiple obstacles and a goal), would never lead to the occurrence of local extrema.

Interestingly, with certain simplifying assumptions, fluid flow properties could be 
derived from a combination of harmonic functions. These functions were part of a well known theory within fluid mechanics called Potential Flow. In Ref. [34], stream functions of potential flow were used to navigate a mobile robot. In Ref. [33], it was shown that by combining potential flow primitives, "panels" defining the boundaries of an obstacle could be created. This methodology is known as the Panel Method in the aerodynamics field.

Kim et al. [33] used obstacles placed in a uniform flow for their avoidance method. Furthermore, a sink was used at the goal point the manipulator was intended to reach. By using the resulting harmonics field, the manipulator successfully avoided obstacles without the local minimum problem arising. However, some parameters of the potential flow method, such as the uniform and sink strength needed to be heuristically chosen and therefore required some trial and error. In Ref. [35], the panel method was employed for multiple robot collision avoidance. Because the "obstacles" were moving robots, the flow parameters needed to be constantly updated and therefore, the parameters could not be set heuristically. Therefore a systematic method was proposed to calculate the flow parameters. Furthermore, in Ref. [36], Fahimi et al. used the panel method for OA of a hyper-redundant manipulator operating in a threedimensional space. In this approach, the two dimensional panel method was expanded to three dimensions and as a result, the manipulator could avoid three-dimensional obstacles.

\subsubsection{Model Predictive Control}

Model Predictive Control (MPC), is a popular method for the control of non-linear dynamic systems $[26,37]$. In this method, an optimal control input sequence is found over a finite receding horizon for a given system with respect to some predefined cost function. The receding horizon is a predictive time horizon over which the cost function is minimized. At each time interval only the first element of the control 
sequence (corresponding to the current time step) is commanded to the system. Then, a new optimal control sequence is determined over a new finite horizon starting at the next time step. Therefore, the horizon keeps shifting forward in time, hence the name receding horizon.

The cost function typically penalizes three occurrences: deviations from the reference input, high control inputs and forbidden states. The appealing feature the method has is that realistic constraints such as input saturation and state constraints can be considered [26]. A drawback of MPC is that the non-linear model of the system is required to determine the receding optimal control. The model required may not always be available or might be very complex. Furthermore, high computation power is required to calculate the optimal control sequence to optimize the cost function over each time interval.

Shim et al. employed MPC to a rotary-wing UAV in Ref. [38]. An artificial potential function was added to the cost function of the MPC method to penalize the approaching of an obstacle. However, because potential functions were used for OA, the method is vulnerable to local minima [39]. Shim et al. succeeded to test the MPC method on a helicopter, but the computation was performed on an on-ground PC and the control inputs were transferred via telemetry to the helicopter. Therefore, this method may not be computationally feasible for real-time onboard computing where computing resources are limited [26].

\subsection{Method Selection}

From the description of the project that was the motivation for this research (Section 1.1), it is clear that the method chosen should have the ability to avoid nonpredefined obstacles in real-time. As explained at the start of this chapter, TP is not of interest and a ROA method should be developed. From Table 2.1, the selected 
method should have a very low response time, a high level of robustness, and should not require high computation resources.

Since the method is to be used on a fixed-wing aircraft, the aircraft's dynamics also need to be accounted for. The aircraft dynamics impose constraints on evasive maneuvers such as the aircraft's minimum turning radius and minimum pull-up radius [40].

The potential scenarios described in Section 1.1.2 suggest that the UAV is not expected to operate in a heavily cluttered environment. For a cluttered environment rotary-wing UAVs should be used as they are more agile than fixed-wing UAVs. In a cluttered environment, an aerodynamically feasible maneuver might not exist for a fixed-wing aircraft. The increased agility of rotary-wing aircraft permits them to perform additional maneuvers such as rapidly decelerating to a stop, hovering, turning while hovering and even flying backwards. In conclusion, the road map and RRT which were developed to perform in cluttered environment are not suitable for the purpose of this research.

The fast responsiveness of the APF method makes it an appealing candidate for fixed-wing ROA. However, even though cluttered environments are not of interest, the UAV should be able to successfully avoid multiple obstacles. The occurrence of local minima in the traditional APF method suggests the use of harmonic potential fields.

Harmonic potential fields representing fluid flow in the potential flow theory are an attractive method for ROA. Using the panel method, obstacles can be inserted in the flow forcing it to avoid the obstacles. This feature makes the panel method and potential flow theory an intuitive and effective method for ROA, which is capable of handling multiple obstacles. The only weakness this method has is its lack of consideration of aircraft dynamics. It will be shown in Section 3.5.2.2, that by appropriately modeling obstacles, the aircraft's dynamic restrictions can be taken into account in 
the panel method.

In conclusion, the use of potential flow functions and the panel method is selected because it has fast response, does not require intensive computation resources, and by means of appropriate obstacle modeling, can account for aircraft constraints. 


\section{Chapter 3}

\section{Theory and Initial Results}

In this chapter, the panel method, which is the chosen method to be used for OA in this research, is introduced and developed. Since the APF method and the usage of harmonics functions were an inspiration to using potential flow theory for OA, they are revisited from the literature review and their strengths and weaknesses are discussed. Further in the chapter, obstacle modeling is discussed. Furthermore, a method to incorporate aircraft constraints in the panel method for obstacle avoidance is introduced.

\subsection{Artificial Potential Field}

The APF was briefly introduced in Section 2.1.3. The method is revisited in this section and the local minima problem is shown in an example.

Consider a scenario where an aircraft (or manipulator) is required to travel to a final goal, while avoiding an obstacle. Repulsive and attractive potential fields $\left(\Phi_{o}\right.$ and $\Phi_{g}$ ) are attached to the obstacle and final goal, respectively. The forces corresponding to these potential fields are defined as

$$
\vec{F}_{o}=-\nabla\left(\Phi_{o}(\vec{x})\right)
$$




$$
\vec{F}_{g}=-\nabla\left(\Phi_{g}(\vec{x})\right)
$$

These artificial forces can be realized by the control system to steer the aircraft around the obstacle and toward the final goal.

It should be noted that the gradient of a scalar field points in the direction of steepest ascent of the field. According to (3.1) and (3.2), $\vec{F}_{o}$ and $\vec{F}_{g}$ point toward the steepest descent directions of their respective potential functions. For an obstacle, the potential function has to be defined in a way that its value increases when approached so that the resulting force will point away from the obstacle, hence a repulsive potential function is used. In contrast, the goal requires an attractive potential with minimum at the goal, so that the resulting force vector will point toward the goal. In Figure 3.1, the combination of an obstacle's repulsive and goal's attractive potential function is shown. Note that the artificial forces are shown in the $x y$ plane and that the obstacle is a two-dimensional circle. The third axis is used to show the APF where large values have been truncated.

The problem of local minima mentioned in section 2.1.3, is shown by assessing the combination of four obstacles. For simplicity, obstacles are restricted to be in a plane, and the potential field of the goal point is neglected. The obstacle potential functions are defined as

$$
\Phi_{i}(\vec{x})=\frac{1}{r_{i}}
$$

where

$$
r_{i}=\sqrt{\left(x-x_{i}\right)^{2}+\left(y-y_{i}\right)^{2}}
$$

and $\left(x_{i}, y_{i}\right)$ is the location of each obstacle. The total potential field is obtained by

$$
\Phi_{\text {total }}=\sum_{i=1}^{4} \Phi_{i}
$$

In Figure 3.2, $\Phi_{\text {total }}$ is plotted for four obstacles located at 


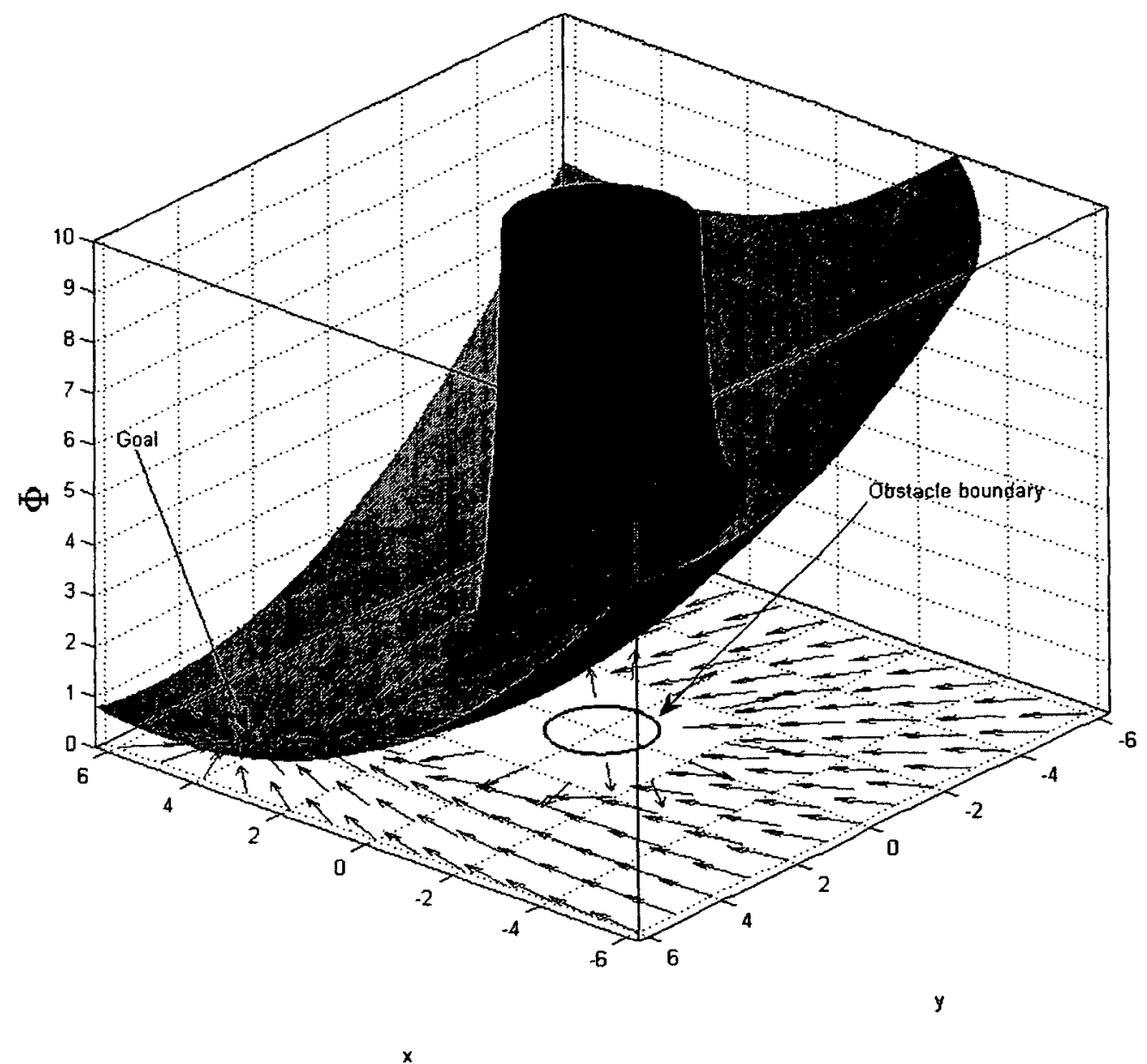

Figure 3.1: APF and artificial forces corresponding to a circle obstacle and a goal 


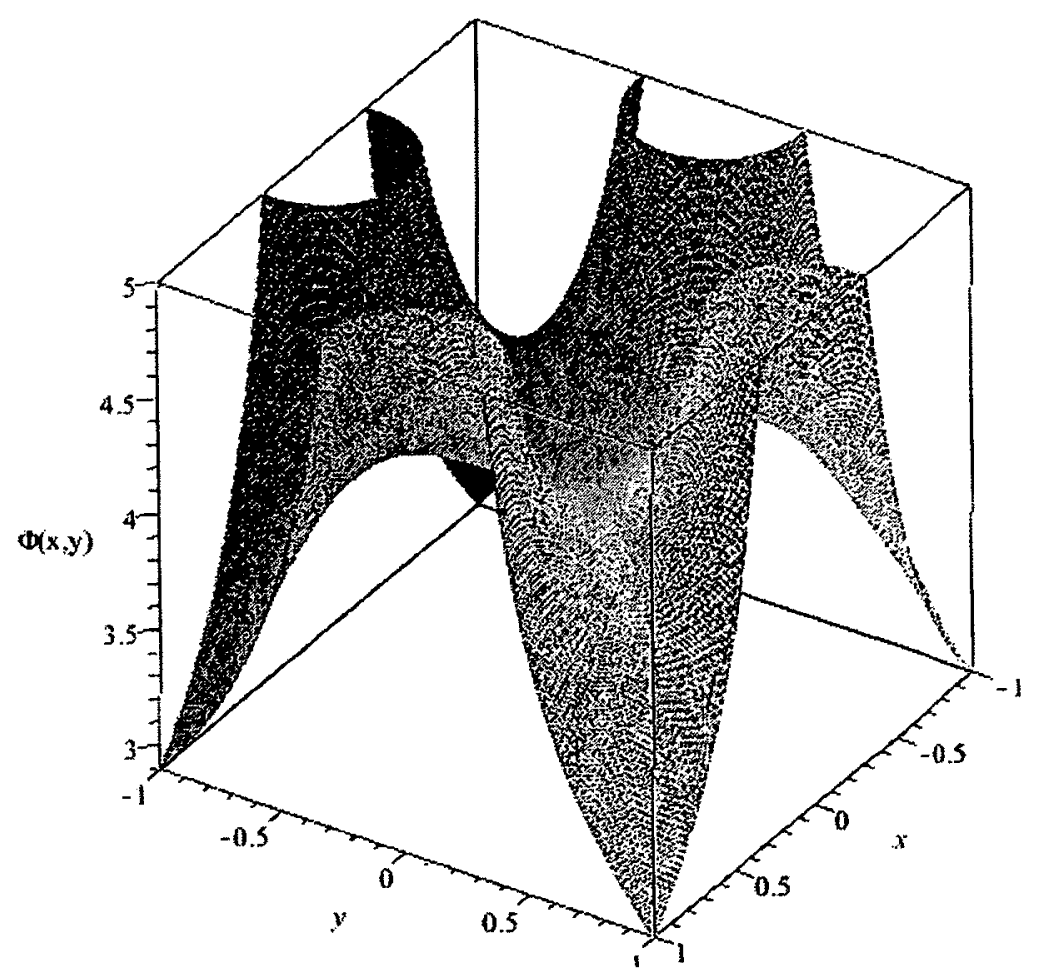

Figure 3.2: The value of four potential functions 
$(1,0),(-1,0),(0,1),(0,-1)$. At $(0,0)$, a local minimum is visible. At this point, the artificial force (gradient of the total potential) is zero and therefore, if a robotic manipulator is being guided, it would be left stranded if it reached that point. In order to avoid the occurrence of local minima, the usage of harmonic functions has been suggested [33].

\subsection{Harmonic Functions}

A harmonic function is a potential function that satisfies the Laplace equation [41], i.e.,

$$
\nabla^{2} \Phi=\overrightarrow{0}
$$

Harmonic functions have two properties that prevent local extrema from occurring when they are added:

1. The superposition property, i.e., any linear combination of harmonic functions is harmonic [42].

2. The corollary of the maximum and minimum principles, i.e., a non-constant function that is harmonic on an open set $\Omega$, does not have extrema in $\Omega[41,42]$.

In conclusion, if multiple obstacles are present, and each obstacle is defined by means of a harmonic potential function, the total potential function will also be harmonic. According to the corollary of the minimum and maximum principle, the total potential function, provided it is non-constant (which it will be by design), will not have local extrema.

A simple function that satisfies the Laplace equation and that is therefore harmonic is

$$
\Phi=-\ln r
$$


where $r=\sqrt{x^{2}+y^{2}}$. In cylindrical coordinates (3.6) is

$$
\nabla^{2} \Phi=\frac{1}{r} \frac{\partial}{\partial r}\left(r \frac{\partial \Phi}{\partial r}\right)+\frac{1}{r^{2}} \frac{\partial^{2} \Phi}{\partial \theta^{2}}+\frac{\partial^{2} \Phi}{\partial z^{2}}
$$

which is zero for $\Phi=-\ln r$.

Returning to the example in Section 3.1, the overall potential function of four harmonics located at $(1,0),(-1,0),(0,1),(0,-1)$ is calculated where

$$
\Phi_{h i}(\vec{x})=-\ln r_{i}
$$

The overall potential is

$$
\Phi_{h_{\text {total }}}=\sum_{i=1}^{4} \Phi_{h i} .
$$

In Figure 3.3, the total of the four harmonic functions are plotted. It can be seen

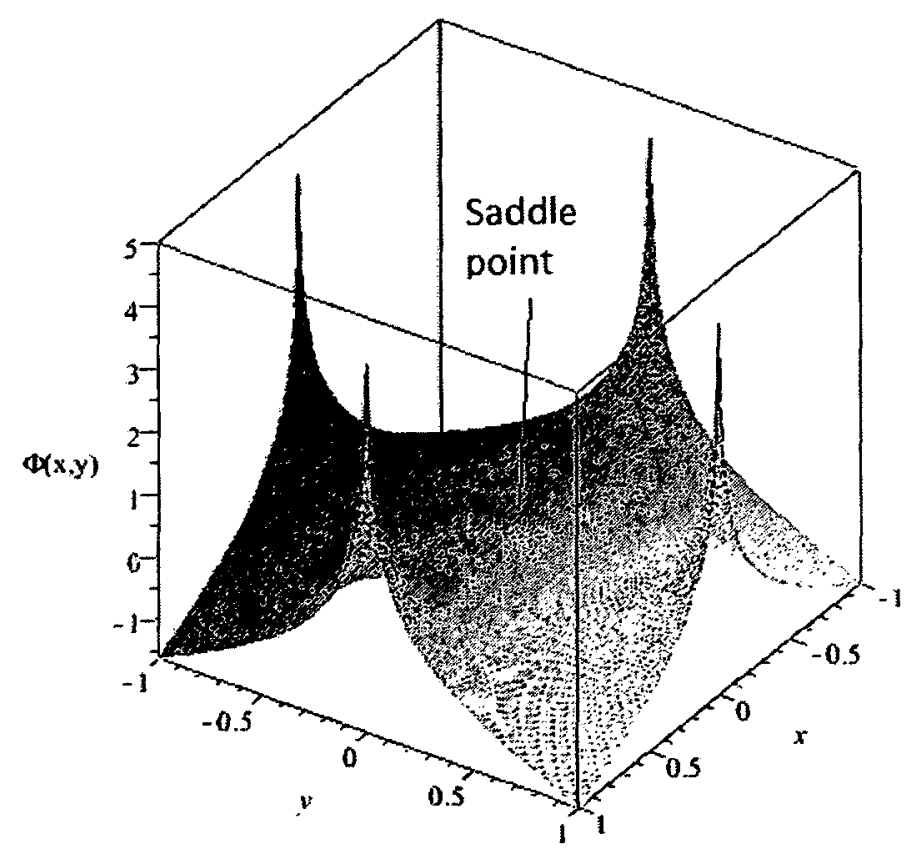

Figure 3.3: The value of four harmonic functions 
that local extrema does not occur'.

At the saddle-point, even though the artificial force is zero, when the manipulator passes this point the artificial force guides it away from the saddle-point. In contrast, the artificial force in the neighborhood of a local minimum points toward the local minimum itself and as a result the manipulator will be commanded to stay at the local minimum.

In the next section it will be demonstrated that, with certain simplifying assumptions, the velocity field of a fluid is the gradient of a harmonic potential function. It will then be demonstrated how potential flow theory from fluid mechanics may be utilized for aircraft OA.

\subsection{Potential Flow}

An inter-disciplinary OA method stemming from fluid mechanics is explained in this section. When a uniform fluid flow encounters an obstacle, fluid particles deviate from their original course and avoid it. This concept can be used for OA, by commanding the aircraft in a way that it would follow the trajectory of a fluid particle (pathline) encountering an obstacle. Since the fluid particles don't flow through obstacles, the aircraft is able to reach its goal without collision.

In fluid mechanics, the Navier-Stokes equations, which govern fluid flow, are derived from conservation of mass, momentum, and energy [43]. However, the complexity of these equations make their usage difficult for $O A$. It will be shown that for an irrotational, inviscid and incompressible flow, a scalar potential function can be found, where its gradient at any point results in the velocity of a fluid particle at that point. Furthermore, it will be shown that this potential function is harmonic and is therefore invulnerable to local extrema. This theory is known as potential flow theory

\footnotetext{
${ }^{1}$ At $(0,0), \Phi_{h_{\text {total }}}$ has a saddle point.
} 
in fluid mechanics ${ }^{2}$.

For irrotational flow

$$
\nabla \times \vec{V}=\overrightarrow{0}
$$

where $\vec{V}$ is the velocity field of the flow. Furthermore, if $\vec{V}$ has continuous partial derivatives, it is a conservative vector field [45], meaning a potential function $\Phi$ exists such that

$$
\vec{V}=\nabla \Phi=\frac{\partial \Phi}{\partial x} \hat{i}+\frac{\partial \Phi}{\partial y} \hat{j}+\frac{\partial \Phi}{\partial z} \hat{k}
$$

and $\hat{i}, \hat{j}$ and $\hat{k}$ are the unit basis vectors pointing along the $x, y$ and $z$ axes, respectively. Assuming incompressible flow, the continuity equation simplifies to

$$
\nabla \cdot \vec{V}=\overrightarrow{0}
$$

Substituting (3.12) into (3.13) yields

$$
\nabla^{2} \Phi=0
$$

which is the Laplace equation and therefore $\Phi$ is harmonic. This guarantees a local extrema free potential function.

\subsubsection{Obstacle Avoidance using Potential Flow}

The velocity field introduced in Section 3.3 is employed to guide a UAV. After entering the velocity field, the aircraft is assumed to be a fluid particle and therefore the velocity at each point is commanded as a course to the aircraft ${ }^{4}$. Therefore, the

\footnotetext{
${ }^{2}$ The potential flow theory is extracted from $[43,44]$

${ }^{3}$ In contrast to the APF method where, $\vec{F}=-\nabla \Phi$, from here on and without loss in generality, $\vec{V}=+\nabla \Phi$ is used.

${ }^{4}$ The course is the direction of travel expressed as an angle in the horizontal plane between a reference line and a tangent to the flight path [46]. See Appendix C.
} 
aircraft will be following a trajectory similar to that of a fluid particle. However, due to autopilot delays, the pathline will not be followed exactly. Note that even though a two-dimensional potential flow is used for obstacle avoidance, the potential flow can be computed in in the horizontal plane (for a fly-around maneuver) and in the vertical plane (for a fly-over maneuver).

In Section 3.4, a method is introduced to obtain the potential field in the presence of obstacles. In Figure 3.4, a velocity field in the presence of an obstacle is illustrated. Note that if the aircraft would be to fly over an obstacle, the velocity at any point would be used as path angle commands ${ }^{5}$. The integration of potential theory and UAV OA will be covered in more detail in Chapters 4 and 5.

Fluid Particle Velocities / Course Commands

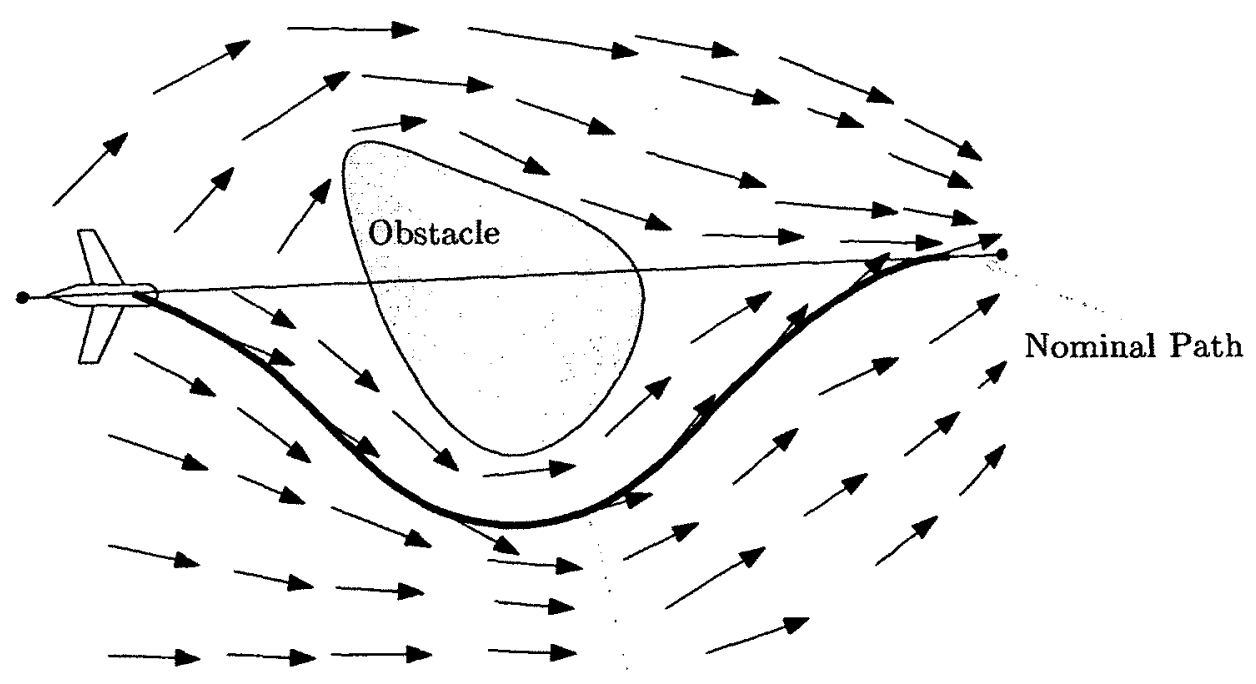

Path Resulted with OA

Figure 3.4: Obstacle avoidance using potential flow

\footnotetext{
${ }^{5}$ The path angle is the angle between the horizontal and a tangent to the flight path [46]. See Appendix C.
} 


\subsubsection{Flow primitives}

In this section, the potential function of three two-dimensional flow primitives that are restricted to the $x y$ plane are derived: uniform flow, sinks and sources. A combination of these primitives will be used to describe more complex flows.

\subsubsection{Uniform Flow}

A uniform steady-state flow is illustrated in Figure 3.5, where

$$
V_{x}=V_{\infty} \cos \alpha \quad, V_{y}=V_{\infty} \sin \alpha
$$

and $V_{\infty}$ is the magnitude of the flow velocity, and $\alpha$ is as illustrated.

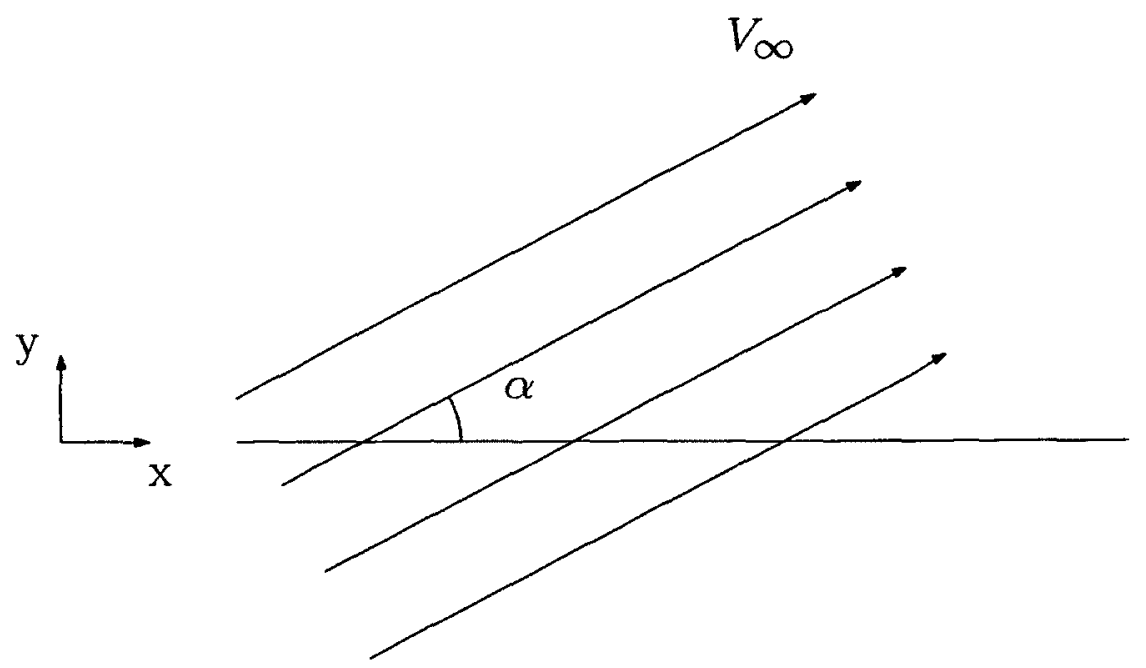

Figure 3.5: Uniform steady-state flow

According to (3.12),

$$
V_{x}=\frac{\partial \Phi_{u}}{\partial x} \quad, V_{y}=\frac{\partial \Phi_{u}}{\partial y}
$$

Therefore, from (3.16), the associated potential function for a uniform flow at an 
angle of $\alpha$ with respect to the $\mathrm{x}$ axis is

$$
\Phi_{u}=\left(V_{\infty} \cos \alpha\right) x+\left(V_{\infty} \sin \alpha\right) y
$$

Note that in (3.17), without loss in generality, the constant of integration has been set to zero.

\subsubsection{Sinks and Sources}

A source is a flow primitive that emits fluid particles radially (Figure 3.6). A sink is the opposite of a source, meaning that it absorbs flow radially. The circumferential velocity of sinks and sources is zero.

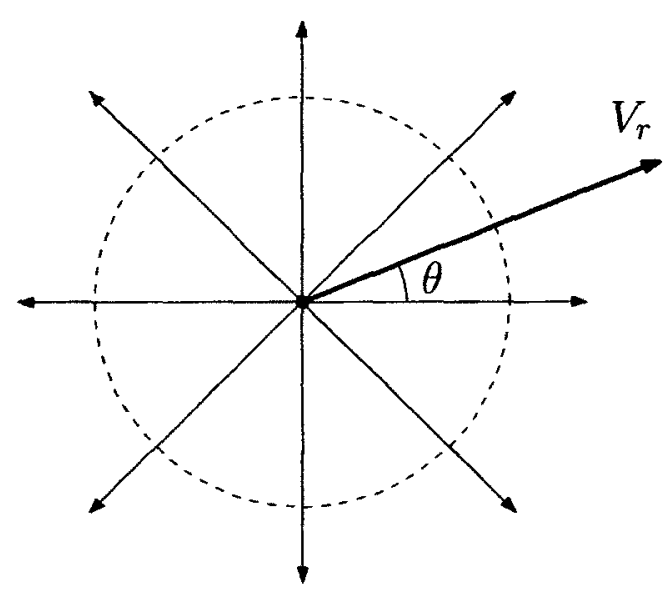

Figure 3.6: Source flow

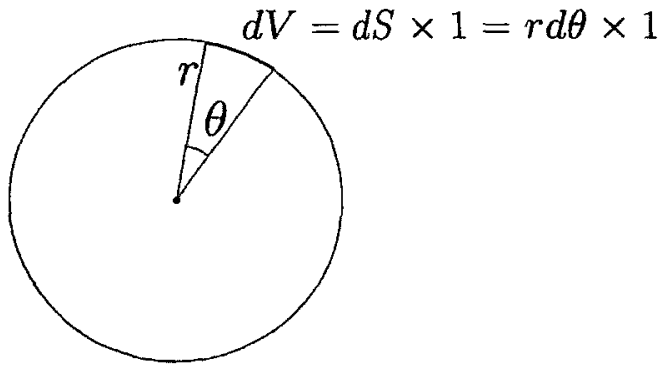

Figure 3.7: Volumetric flow from a source

In cylindrical coordinates $(x=r \cos \theta, y=r \sin \theta)$, for two-dimensional flow, (3.12) changes to

$$
\vec{V}=\nabla \Phi=\frac{\partial \Phi}{\partial r} \hat{e}_{r}+\frac{1}{r} \frac{\partial \Phi}{\partial \theta} \hat{e}_{\theta}
$$

where $\hat{e}_{r}$ and $\hat{e}_{\theta}$ are the unit basis vectors pointing along the $r$ and $\theta$ coordinates, respectively. Therefore for two-dimensional flow, the radial component of the flow 
velocity is

$$
V_{r}=\frac{\partial \Phi}{\partial r}
$$

and the tangential component is

$$
V_{\theta}=\frac{1}{r} \frac{\partial \Phi}{\partial \theta}
$$

noting that $V_{\theta}=0$, since flow is purely radial, as shown in Figure 3.6. From (3.20) it must be that

$$
\frac{\partial \Phi}{\partial \theta}=0 \Rightarrow \Phi=f(r)
$$

On the other hand $\Phi$ has to be harmonic. Therefore from (3.8)

$$
\frac{1}{r} \frac{\partial}{\partial r}\left(r \frac{\partial \Phi}{\partial r}\right)=0 \Rightarrow \frac{\partial}{\partial r}\left(r \frac{\partial \Phi}{\partial r}\right)=0 \Rightarrow r \frac{\partial \Phi}{\partial r}=g(\theta)
$$

Therefore

$$
\Phi=g(\theta) \ln r
$$

From (3.21) and (3.23), it is concluded that $g(\theta)=c$ and

$$
\Phi=c \ln r
$$

where $c$ is constant.

The source/sink is assumed to have a volumetric flow per unit of depth (in the $z$ direction), $\Lambda$. From Figure 3.7

$$
\Lambda=\oint V_{r} d V=\int_{0}^{2 \pi}\left(\frac{c}{r}\right) r d \theta \times 1=2 \pi c
$$


Therefore

$$
\Phi_{s}=\frac{\Lambda}{2 \pi} \ln r \quad 6
$$

where $\Lambda$ is the source/sink strength and is positive for a source and negative for a sink.

\subsection{Panel Method}

The flow primitives introduced in Section 3.3.2, can be directly used to define simple flow cases, e.g. a uniform flow being sunk at a point, or a source emitting flow and a sink absorbing it. However, to model obstacles within a uniform flow, a more complex combination of flow primitives is used. Obstacles are modeled by a set of straight panels. These panels are created by combining an infinite number of infinitesimally small sources and sinks. The source and sink strengths are chosen in a way that the velocity normal to each panel is forced to zero on the panel itself. As a result, the flow will not traverse the panels, and the flow avoids the obstacle. This method is known as the panel method. The following discussion of the panel method is taken from Ref. [44].

The potential for a single panel is defined first. For an infinitesimally small source the potential is (compare to (3.26))

$$
d \Phi=\frac{\lambda}{2 \pi} \ln (r) d s
$$

where $\lambda$ is the source strength per unit of depth and unit of length of each panel ${ }^{7}$. In Figure 3.8, a panel created from an unlimited number of sources/sinks is illustrated. The total potential of this panel is

\footnotetext{
${ }^{6} \Lambda$ 's unit is $m^{2} / s$

${ }^{7} \lambda$ 's unit is $\mathrm{m} / \mathrm{s}$
} 


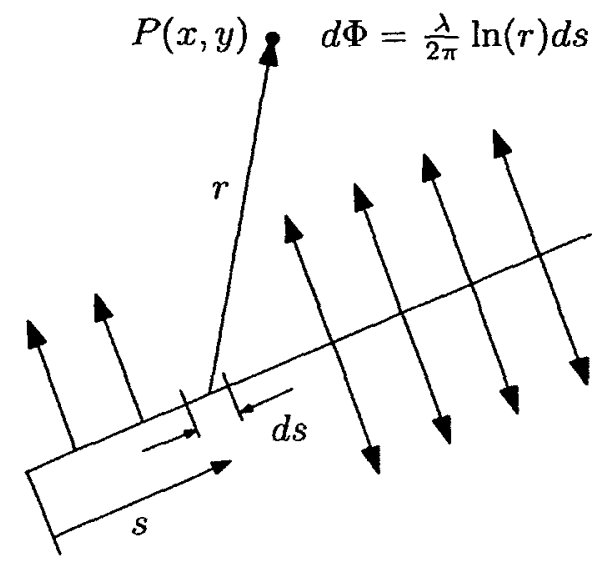

Figure 3.8: Source sheet

$$
\Phi(x, y)=\int_{0}^{S} \frac{\lambda}{2 \pi} \ln r d s
$$

where $S$ is the length of the panel. The panel is assumed to have constant strength over its length. Therefore

$$
\Phi(x, y)=\frac{\lambda}{2 \pi} \int_{0}^{S} \ln r d s
$$

In Figure 3.9, a closed obstacle with $n$ panels is immersed in a uniform flow. From (3.29), the potential due to the $j^{\text {th }}$ panel at $\mathrm{P}$ is

$$
\Phi_{j}=\frac{\lambda_{j}}{2 \pi} \int_{0}^{S_{j}} \ln r_{P j} d s_{j}
$$

where $\lambda_{j}$ is the source strength per unit of length for the $j^{\text {th }}$ panel and

$$
r_{P j}=\sqrt{\left(x-x_{j}\right)^{2}+\left(y-y_{j}\right)^{2}}
$$

Note that $\left(x_{j}, y_{j}\right)$ are running coordinates over the $j^{\text {th }}$ panel and that $S_{j}$ is the length of the $j^{\text {th }}$ panel. 


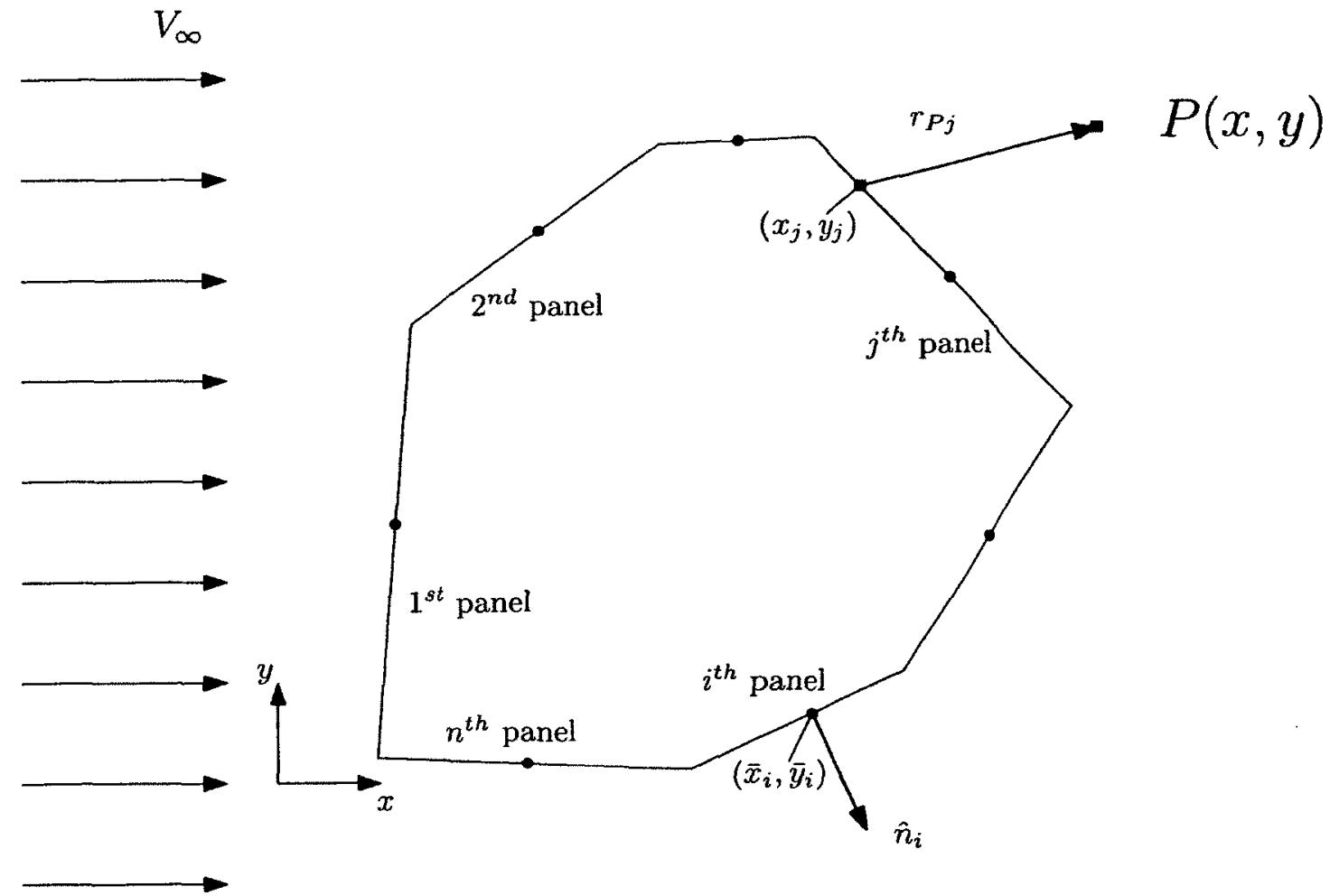

Figure 3.9: An n-panel obstacle 
The potential due to all panels is

$$
\Phi_{o}(P)=\sum_{j=1}^{n} \frac{\lambda_{j}}{2 \pi} \int_{0}^{S_{j}} \ln r_{P_{j}} d s_{j}
$$

where $\Phi_{o}$ is the potential function of the obstacle.

The midpoint of each panel, $\left(\bar{x}_{i}, \bar{y}_{i}\right)$, is chosen as a control point and the velocity perpendicular to the panels is forced to zero at these points. Therefore at the $i^{t h}$ control point, one must have (by superposition of flows),

$$
V_{\infty\left(n_{i}\right)}+V_{\left(n_{i}\right)}=0
$$

where $V_{\infty_{\left(n_{i}\right)}}$ is the normal component of free-stream velocity to the $i^{\text {th }}$ panel and $V_{\left(n_{i}\right)}$ is the normal component of the velocity due to the potential corresponding to all panels at the $i^{\text {th }}$ control point. The free-stream component is obtained from

$$
V_{\infty\left(n_{i}\right)}=\vec{V}_{\infty} \cdot \hat{n}_{i}
$$

where $\hat{n}_{i}$ is the unit outward pointing normal for the $i^{\text {th }}$ panel, and $\vec{V}_{\infty}$ is the freestream velocity vector.

The potential due to the obstacle at the $i^{\text {th }}$ control point is obtained by setting $\mathrm{P}$ to $\left(\bar{x}_{i}, \bar{y}_{i}\right)$ in $(3.32)$. Therefore

$$
\Phi_{o}\left(\bar{x}_{i}, \bar{y}_{i}\right)=\sum_{j=1}^{n} \frac{\lambda_{j}}{2 \pi} \int_{0}^{S_{j}} \ln r_{i j} d s_{j}
$$

where

$$
r_{i j}=\sqrt{\left(\bar{x}_{i}-x_{j}\right)^{2}+\left(\bar{y}_{i}-y_{j}\right)^{2}}
$$


and consequently,

$$
V_{n_{i}}=\frac{\partial \Phi\left(\bar{x}_{i}, \bar{y}_{i}\right)}{\partial n_{i}}=\sum_{j=1}^{n} \frac{\lambda_{j}}{2 \pi} \int_{0}^{S_{j}} \frac{\partial}{\partial n_{i}} \ln r_{i j} d s_{j}
$$

Note that $\partial f / \partial n_{i}$ is used as a shorthand for $\nabla f \cdot \hat{n}_{i}$. Moreover, recall that $\left(x_{j}, y_{j}\right)$ are running coordinates over the $j^{\text {th }}$ panel.

In (3.37), for $j=i, \ln \left(r_{i j}\right)$ is singular at $\left(x_{i}, y_{i}\right)=\left(\bar{x}_{i}, \bar{y}_{i}\right)$. The contribution of the $i^{\text {th }}$ panel to $V_{n_{i}}$ is derived as a special case. Without loss in generality the $i^{\text {th }}$ panel is shown in Figure 3.10 with some simplification. From Figure 3.10, initially letting

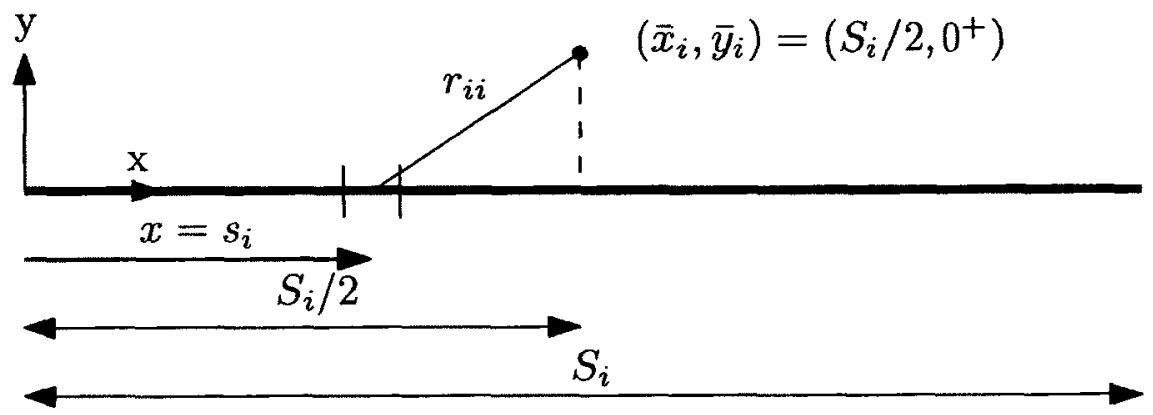

Figure 3.10: Computing the contribution of the $i^{\text {th }}$ panel to $V_{n_{i}}$

$y>0$, one has

$$
\begin{aligned}
\frac{\lambda_{i}}{2 \pi} \int_{0}^{S_{i}} \frac{\partial}{\partial n_{i}} \ln r_{i i} d s_{i} & =\frac{\lambda_{i}}{2 \pi} \int_{0}^{S_{i}} \frac{\partial}{\partial y} \ln \left(\sqrt{\left(\frac{S_{i}}{2}-x\right)^{2}+y^{2}}\right) d x \\
& =-\frac{\lambda_{i}}{2 \pi}\left(\arctan \frac{-S_{i}}{2 y}-\arctan \frac{S_{i}}{2 y}\right)\left[47, \text { p. 64], letting } y \rightarrow 0^{+}\right. \\
& =-\frac{\lambda_{i}}{2 \pi}\left(-\frac{\pi}{2}-\frac{\pi}{2}\right) \\
& =\frac{. \lambda_{i}}{2}
\end{aligned}
$$

Therefore (3.35) is

$$
V_{n_{i}}=\frac{\lambda_{i}}{2}+\sum_{\substack{j=1 \\ j \neq i}}^{n} \frac{\lambda_{j}}{2 \pi} I_{i, j}
$$


where

$$
I_{i, j}=\int_{0}^{S_{j}} \frac{\partial}{\partial n_{i}} \ln r_{i j} d s_{j}
$$

Note that an expression for evaluating (3.40) will be given in Section 3.4.1.

Deriving this equation for all control points and combining it with (3.33) leads to

$$
\left[\begin{array}{ccc}
\frac{1}{2} & \cdots & \frac{I_{i, j}}{2 \pi} \\
\vdots & \ddots & \vdots \\
\frac{I_{i, j}}{2 \pi} & \cdots & \frac{1}{2}
\end{array}\right]\left[\begin{array}{c}
\vdots \\
\lambda_{i} \\
\vdots
\end{array}\right]=\left[\begin{array}{c}
\vdots \\
-\vec{V}_{\infty} \cdot \hat{n}_{i} \\
\vdots
\end{array}\right]
$$

or

$$
[I][\vec{\lambda}]=\left[-\vec{V}_{\infty_{n}}\right]
$$

where the definitions of the quantities in (3.42) are obvious from (3.41). Note that (3.42) is a system of $n$ equations and $n$ variables, where $n$ is the number of panels of the obstacle. By solving (3.42), the required panel strengths $\lambda_{1}, \lambda_{2}, \ldots, \lambda_{n}$ are obtained.

The potential for any point $P$ in the flow, is the sum of the uniform potential, $\Phi_{u}$ and the obstacle potential, $\Phi_{o}$. Therefore,

$$
\Phi_{T}=\Phi_{u}+\Phi_{o} .
$$

From (3.17) with $\alpha=0$ and (3.32)

$$
\Phi_{T}=V_{\infty} x+\sum_{j=1}^{n} \frac{\lambda_{j}}{2 \pi} \int_{0}^{S_{j}} \ln r_{P j} d s_{j}
$$

where

$$
r_{P_{j}}=\sqrt{\left(x-x_{j}\right)^{2}+\left(y-y_{j}\right)^{2}} .
$$


Finally, from $(3.12)$ the total velocity field of an obstacle immersed in a twodimensional uniform flow is

$$
V_{x}(x, y)=V_{\infty}+\sum_{j=1}^{n} \frac{\lambda_{j}}{2 \pi} J_{x, j}
$$

and

$$
V_{y}(x, y)=\sum_{j=1}^{n} \frac{\lambda_{j}}{2 \pi} J_{y, j}
$$

where

$$
J_{x, j}=\int_{0}^{S_{j}} \frac{\partial}{\partial x} \ln r_{P j} d s_{j}
$$

and

$$
J_{y, j}=\int_{0}^{S_{j}} \frac{\partial}{\partial y} \ln r_{P_{j}} d s_{j}
$$

Expressions for evaluating $J_{x, j}$ and $J_{y, j}$ are presented in Section 3.4.1.

In the panel method presented above, the source strengths were assumed to be constant over each panel and the velocity was forced to zero at only one control point on each panel. These simplifications affect the accuracy to which the computed potential function approximates the fluid flow. To measure the accuracy, the amount of fluid being added or absorbed from the flow is used. For a closed body this value, which will be called "obstacle strength",

$$
\sum_{j=1}^{n} \lambda_{j} S_{j}
$$

should be equal to zero.

The potential function is accurate at the control points. But further away from the control points, the potential function is more prone to error. This error may lead to flow entering the obstacle. Therefore if such a potential function is used for obstacle avoidance for an aircraft, the aircraft could collide with obstacles (since there 
would be pathlines entering it).

By increasing the number of panels, i.e. increasing the number of control points, error is decreased to a point where an infinite number of panels guarantees no error and therefore no flow entering the obstacle. The effect of increasing the number of panels is further discussed in Section 3.5.1.1.

In Figure 3.11, the velocity field of a unit square immersed in a uniform horizontal flow is plotted on a grid. The particle trajectories (the pathlines) are tangent to the velocity vector at any point. It should be noted that the potential function is not valid for points within the obstacle because flow never enters the obstacle and fluid particle velocities within the obstacle are meaningless. Therefore the velocity vectors inside the obstacle must be neglected. As explained in Section 3.3.1 and in Figure 3.4, the velocity vector at each point can be used as a course/path angle command to direct the aircraft away from the obstacle.

\subsubsection{Calculating $I_{i, j}, J_{x, j}, J_{y, j}$}

In Figure 3.12 the $i^{\text {th }}$ and $j^{\text {th }}$ panels from Figure 3.9 are illustrated. The panel endpoints and mid-panel control points are enumerated clockwise as $\left(X_{j}, Y_{j}\right)$ and $\left(\bar{x}_{j}, \bar{y}_{j}\right)$ respectively. $\varphi_{j}$ is the angle of each panel from the $x$ axis in the counterclockwise direction. $\beta_{j}$ is the angle between $\hat{n}_{j}$ and the $x$ axis in the counterclockwise direction. $s_{j}$ is distance measured along each panel from $\left(X_{j}, Y_{j}\right)$. Finally, $S_{j}$ is the length of the $j^{\text {th }}$ panel.

From Figure 3.12,

$$
\begin{aligned}
\beta_{j} & =\varphi_{j}+\frac{\pi}{2} \\
x_{j} & =X_{j}+s_{j} \cos \varphi_{j} \\
y_{j} & =Y_{j}+s_{j} \sin \varphi_{j} \\
\hat{n}_{j} & =\left(\cos \beta_{j}, \sin \beta_{j}\right)
\end{aligned}
$$




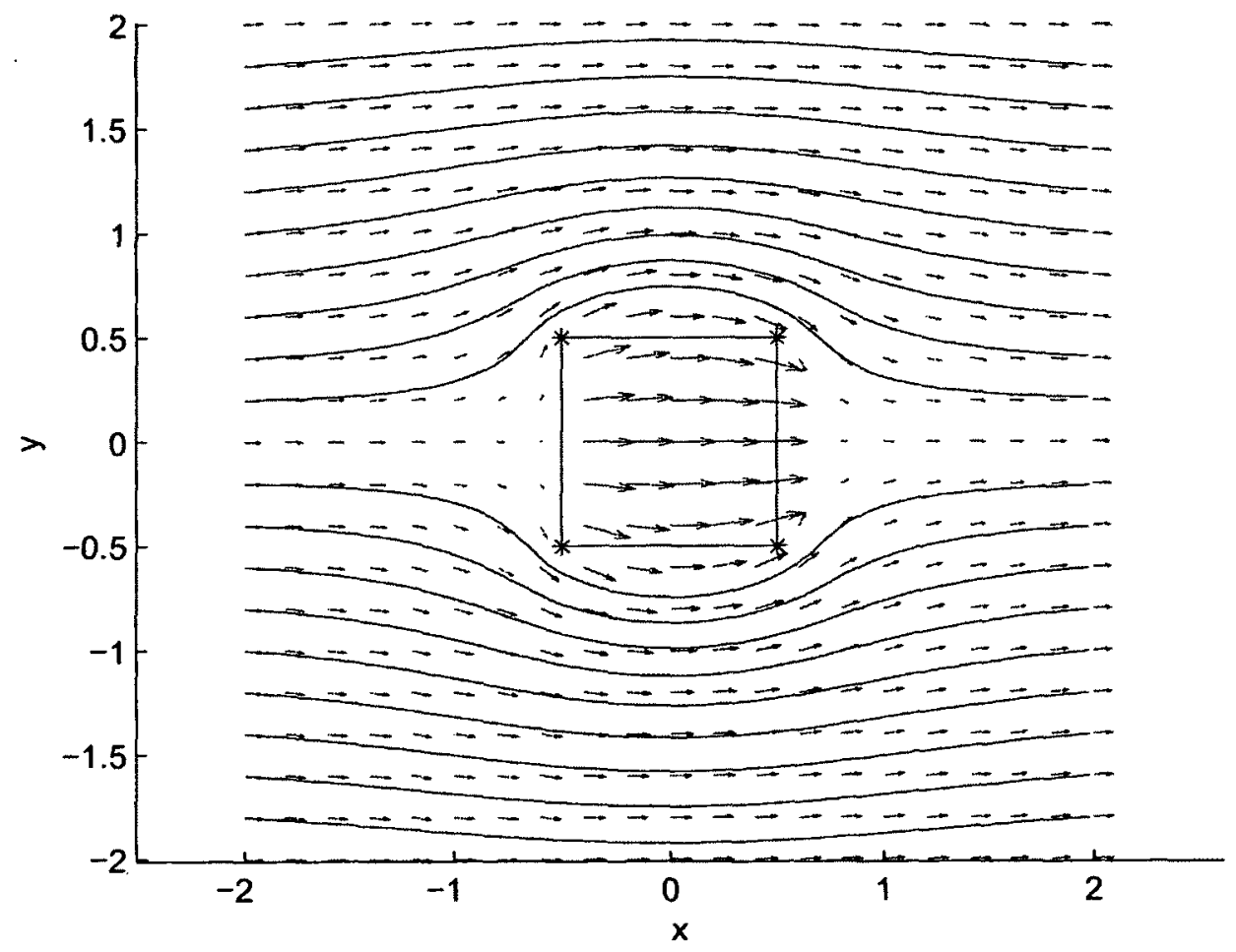

Figure 3.11: Velocity field and flow pathlines around a unit square. $V_{\infty}=1 \mathrm{~m} / \mathrm{s}$

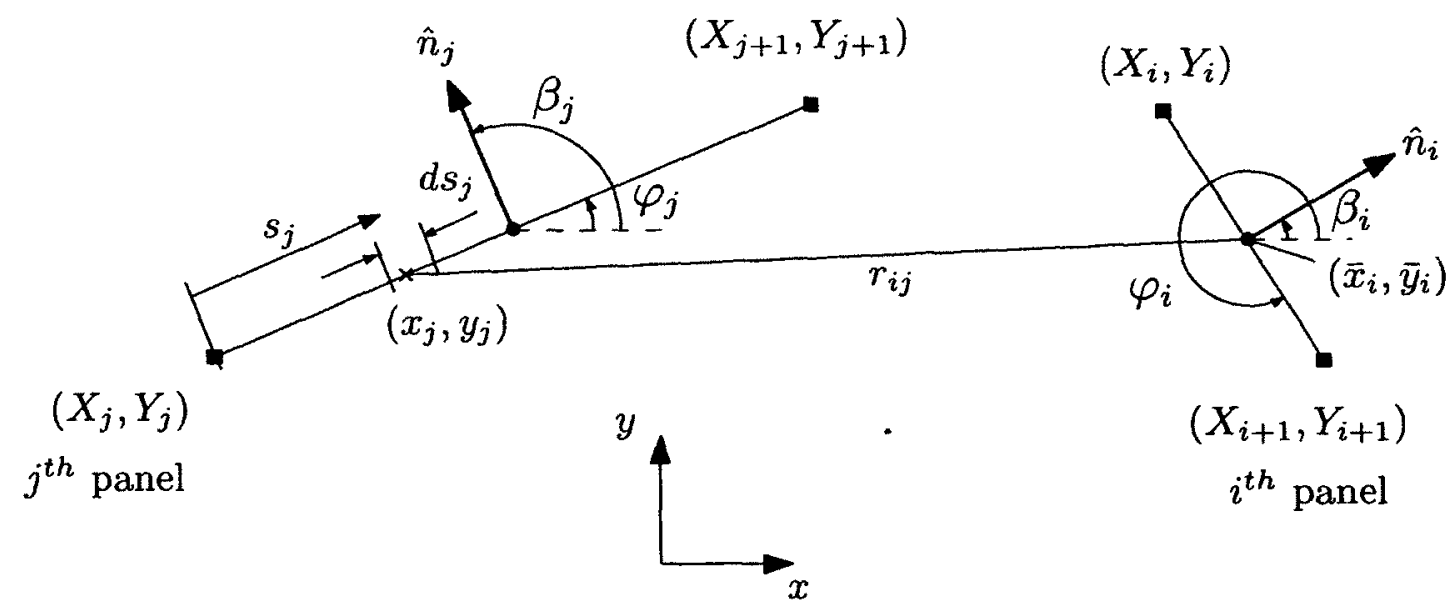

Figure 3.12: Calculating $I_{i, j}$ 
where $\left(x_{j}, y_{j}\right)$ are running coordinates over the $j^{\text {th }}$ panel. Solving (3.40) using (3.51) results in,

$$
I_{i, j}=\int_{0}^{S_{j}} \frac{C s_{j}+D}{s_{j}^{2}+2 A s_{j}+B} d s_{j}
$$

where

$$
\begin{aligned}
& A=-\left(\bar{x}_{i}-X_{j}\right) \cos \varphi_{j}-\left(\bar{y}_{i}-Y_{j}\right) \sin \varphi_{j} \\
& B=\left(\bar{x}_{i}-X_{j}\right)^{2}+\left(\bar{y}_{i}-Y_{j}\right)^{2} \\
& C=\sin \left(\varphi_{i}-\varphi_{j}\right) \\
& D=\left(\bar{y}_{i}-Y_{j}\right) \cos \varphi_{i}-\left(\bar{x}_{i}-X_{j}\right) \sin \varphi_{i}
\end{aligned}
$$

Therefore from $[47, \mathrm{p} .67]$

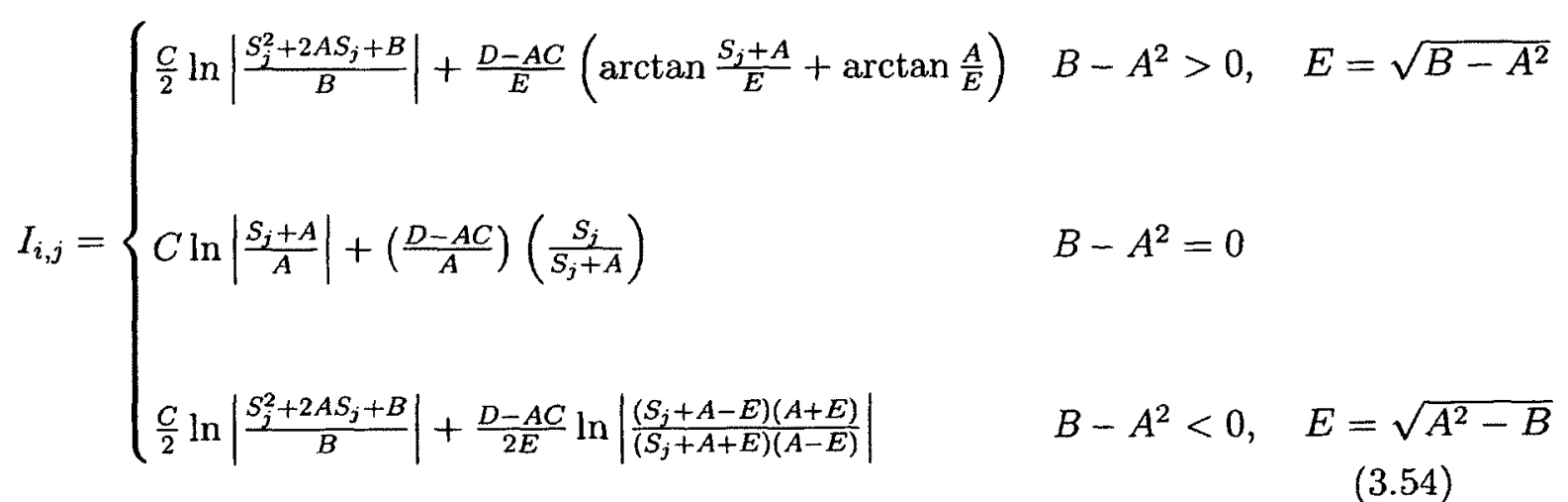

Similarly $J_{x, j}$ and $J_{y, j}$ are derived from (3.48) and (3.49). They can both be 
calculated from

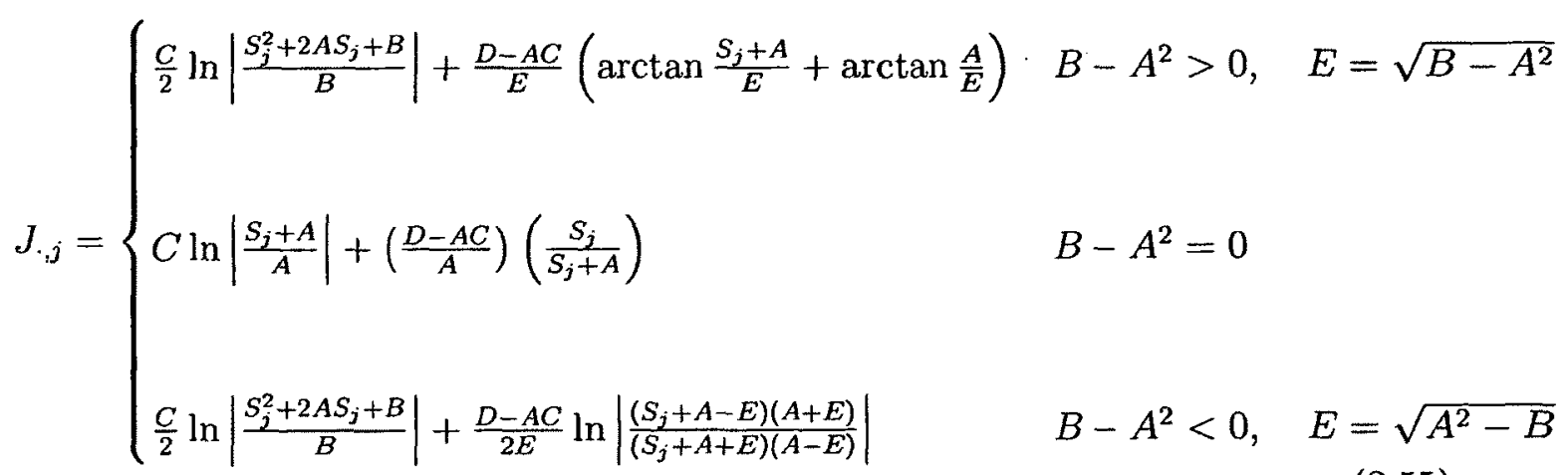

where

$$
\begin{aligned}
& A=\left(X_{j}-x\right) \cos \varphi_{j}+\left(Y_{j}-y\right) \sin \varphi_{j} \\
& B=\left(x-X_{j}\right)^{2}+\left(y-Y_{j}\right)^{2}
\end{aligned}
$$

and for $J_{x, j}$

$$
\begin{aligned}
& C=-\cos \varphi_{j} \\
& D=x-X_{j}
\end{aligned}
$$

and for $J_{y, j}$

$$
\begin{aligned}
& C=-\sin \varphi_{j} \\
& D=y-Y_{j}
\end{aligned}
$$

\subsection{Obstacle Modeling \& Implementation}

\subsubsection{Obstacle Modeling}

In Section 1.1.3, the OD research and method were explained. It was stated that OD detects the boundaries of obstacles within a two dimension vertical plane in front of the UAV, detects the depth of the vertical plane and defines a rectangle surrounding the obstacles in that plane. Therefore, the OD method does not initially give any detail about the depth of the obstacle. Because there is no information about the third dimension of the obstacle, it is initially assumed to have a depth equal to its 
width (Figure 3.13 and 3.14).
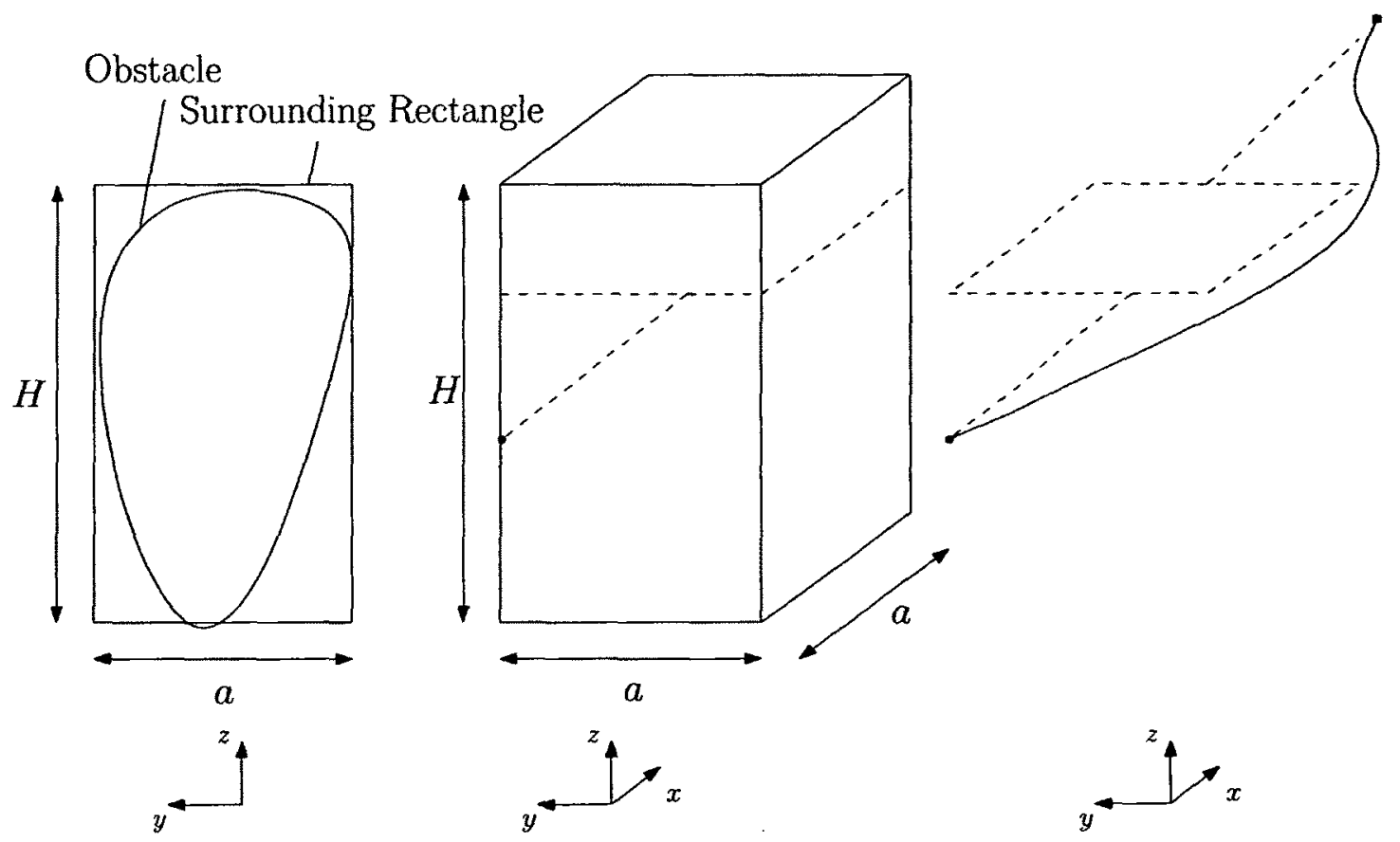

Figure 3.13: Assuming equal depth and width in obstacle modeling

As the aircraft starts the avoidance maneuver and newer OD data becomes available, the depth of the obstacle is updated such as in Figure 3.15. Note that the uniform free-stream flow direction required for the panel method is set to the UAV's original course of flight.

If the exact contour of an obstacle is known, the panel method creates velocity fields that follow the obstacles more closely than with the equal depth and width assumption.

\subsubsection{Increasing the Number of Panels}

In the panel method presented, the value of source strength was assumed to be constant over each panel. Furthermore, the velocity was forced to zero at only one control point on each panel. Therefore, the farther a point is away from the control point, the 


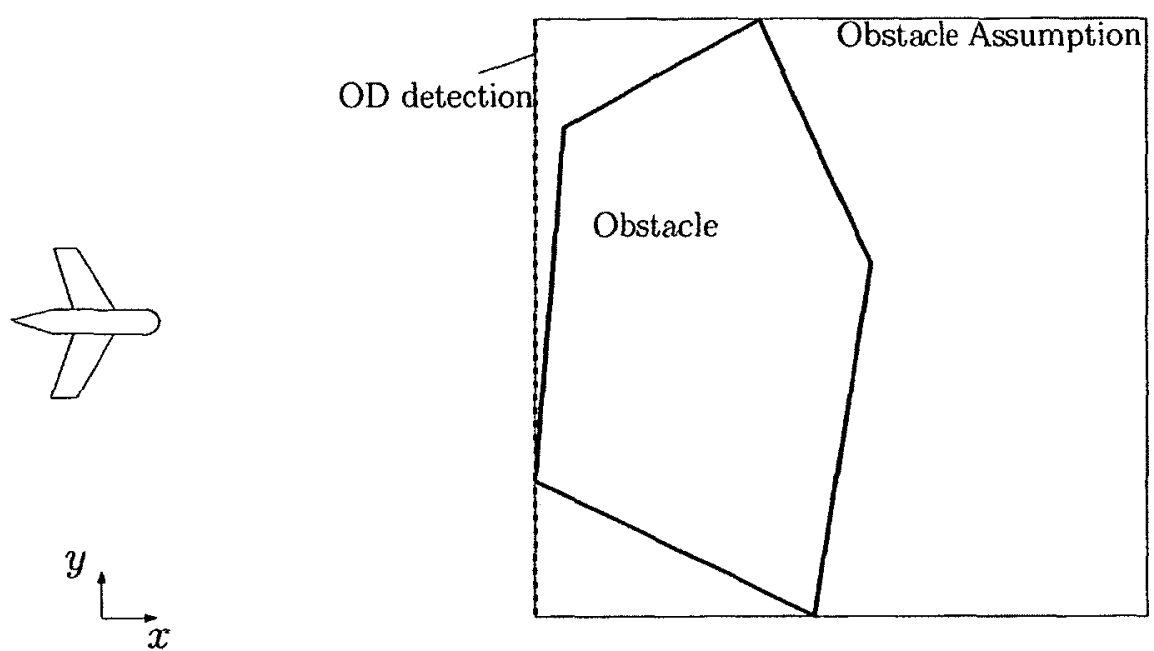

Figure 3.14: Difference between exact contour and assumption based on equal width and depth

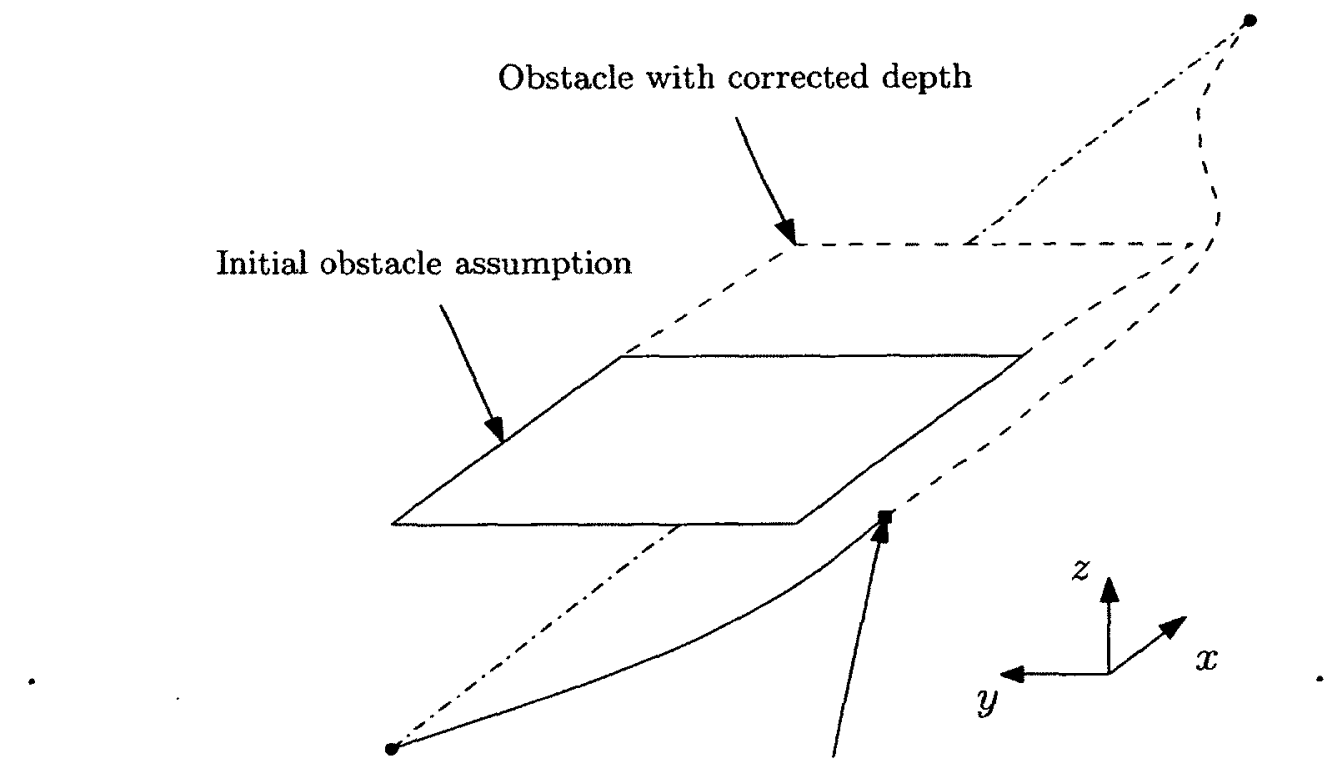

Point where obstacle shape is updated and new potential function is calculated

Figure 3.15: Obstacle being updated 


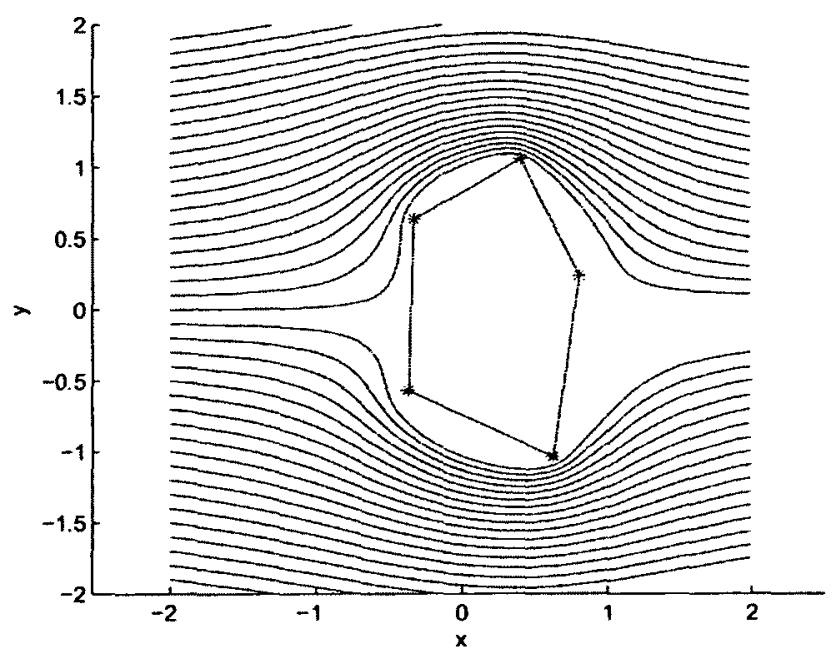

Figure 3.16: Avoiding pathlines for a random polygon

higher is the possibility of the flow entering the obstacle at that point. By increasing the number of panels, i.e. increasing the number of control points, the accuracy of the potential function is increased, to a point where an infinite number of panels guarantees no flow entering the obstacle.

However, increasing the number of panels requires additional computation time. Firstly, the size of the system of equations, (3.41), increases and therefore a longer time is required to calculate the panel strengths. Secondly, the number of terms in the velocity field function (equations (3.46) and (3.47)) increases. This means that the velocity will take longer to be calculated. In order to compare the accuracy of the potential function for different panel numbers, the obstacle strength (given by (3.50)) is computed. The closer its value is to zero, the better the accuracy of the potential function is.

The potential function for a random polygon, shown in Figure 3.16, was calculated for different numbers of sub-panels. In Table 3.1, the computation time used and the obstacle strength is summarized.

The fourth column of this table is the time used to compute the panel strength 
values from (3.41). In the fifth column, the time used to compute $\left[V_{x}(x, y), V_{y}(x, y)\right]$ at a given point from (3.46) and (3.47), after the panel strengths have been computed, is stated. Note that the panel strengths need to be computed only once when the obstacle is detected (or when new obstacle information is available), whereas the velocity needs to be re-computed at each sample instant at the aircraft's location, as the aircraft travels through the velocity field. Also, the computation time was averaged for five trials ${ }^{8}$.

In Table 3.1, it is seen that an increase in the number of sub-panels, increases the accuracy of the calculated potential function (the obstacle strength reduces), but at the expense of longer computation.

The time required to calculate the velocity is reasonable for any number of subpanels in Table 3.1. However, the time required to calculate the panel strengths may be infeasible for real-time applications past 10 sub-panels. OD was shown to be capable of detecting obstacles up to $784 m$ away (for an obstacle with $1.2 m$ width) [10]. Considering an aircraft flying with a ground speed of 200 knots (in reality the UAVs of interest fly at much lower speeds), suggests that with 10 sub-panels, the aircraft would fly a distance of less than $10 \mathrm{~m}$ while the velocity field is being computed. Therefore the aircraft would still have a safe distance with the obstacle to successfully avoid it $(>774 m)$.

The reason why computing the panel strengths is very lengthy is that in order to solve (3.41) an $n \times n$ system of equations needs to be solved ( $n$ is the total number of panels), which is time consuming.

In Figure 3.17, the velocity field around a concave obstacle has been plotted. In Figure 3.18, the concave region is magnified where it can be seen that the calculated velocity field is inaccurate and the flow is directed to enter the obstacle. To increase the accuracy of the velocity field, each panel is broken down to 10 sub-panels

\footnotetext{
${ }^{8}$ Computation was done on a Core i5 CPU using a single $2.5 \mathrm{GHz}$ core.
} 


\begin{tabular}{|c|c|c|c|c|}
\hline $\begin{array}{c}\text { Number of } \\
\text { Sub-panels } \\
\text { for Each Panel }\end{array}$ & $\sum_{j=1}^{n} \lambda_{j} S_{j}$ & Trial No. & $\begin{array}{l}\text { Time to Calculate } \\
\text { Panel Strengths (s) }\end{array}$ & $\begin{array}{c}\text { Time to Calculate } \\
\text { Velocity of } \\
\text { One Point (s) }\end{array}$ \\
\hline \multirow{6}{*}{1} & \multirow{6}{*}{0.1731} & 1 & 0.0618 & $5.20 \mathrm{E}-05$ \\
\hline & & 2 & 0.0828 & $7.08 \mathrm{E}-05$ \\
\hline & & 3 & 0.0637 & $5.24 \mathrm{E}-05$ \\
\hline & & 4 & 0.0834 & $6.84 \mathrm{E}-05$ \\
\hline & & 5 & 0.0700 & $5.63 \mathrm{E}-05$ \\
\hline & & Average & 0.0723 & $6.00 \mathrm{E}-05$ \\
\hline \multirow{6}{*}{10} & \multirow{6}{*}{0.0145} & 1 & 0.0847 & $1.10 \mathrm{E}-04$ \\
\hline & & 2 & 0.0848 & $1.15 \mathrm{E}-04$ \\
\hline & & 3 & 0.0872 & 1.37E-04 \\
\hline & & 4 & 0.0880 & $1.31 \mathrm{E}-04$ \\
\hline & & 5 & 0.0646 & $1.12 \mathrm{E}-04$ \\
\hline & & Average & 0.0819 & $1.11 \mathrm{E}-04$ \\
\hline \multirow{6}{*}{100} & \multirow{6}{*}{0.0019} & 1 & 0.3880 & $8.74 \mathrm{E}-04$ \\
\hline & & 2 & 0.4987 & $7.89 \mathrm{E}-04$ \\
\hline & & 3 & 0.3190 & $7.73 \mathrm{E}-04$ \\
\hline & & 4 & 0.3216 & $6.82 \mathrm{E}-04$ \\
\hline & & 5 & 0.4011 & $8.74 \mathrm{E}-04$ \\
\hline & & Average & 0.3857 & $7.98 \mathrm{E}-04$ \\
\hline \multirow{6}{*}{1000} & \multirow{6}{*}{$1.60 \mathrm{E}-05$} & 1 & 30.8224 & $5.90 \mathrm{E}-03$ \\
\hline & & 2 & 26.4100 & $2.60 \mathrm{E}-03$ \\
\hline & & 3 & 28.9441 & $6.50 \mathrm{E}-03$ \\
\hline & & 4 & 30.0297 & 8.20E-03 \\
\hline & & 5 & 25.6227 & $2.10 \mathrm{E}-03$ \\
\hline & & Average & 28.3658 & $5.06 \mathrm{E}-03$ \\
\hline
\end{tabular}

Table 3.1: Computing time for different number of panels 
(Figure 3.19). In this case, the flow behaves correctly and does not enter the obstacle.

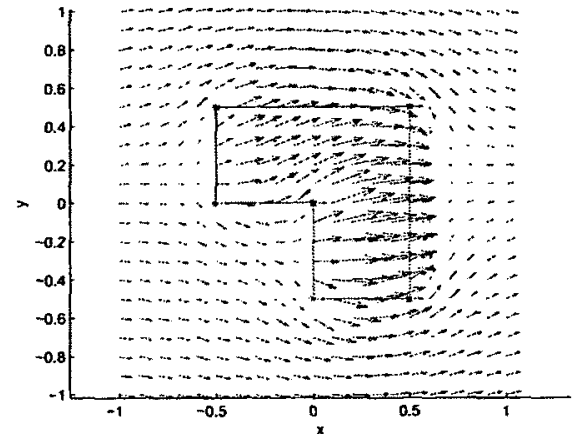

Figure 3.17: Concave obstacle

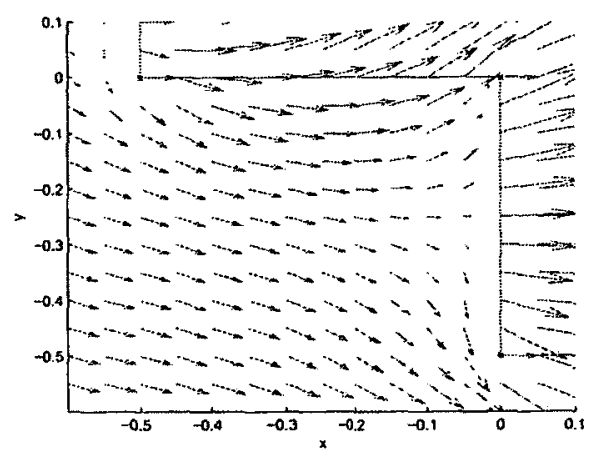

Figure 3.18: Concave obstacle (concave region is magnified)

\subsubsection{Incorporating Aircraft Constraints}

The velocity field obtained in Section 3.4 does not take aircraft dynamics into account. In this section, aircraft constraints are introduced from [40], and a method to incorporate these constraints in the potential flow method is proposed.

\subsubsection{Aircraft Constraints}

A limitation fixed-wing aircraft have is the tightest turn they can make while maintaining level flight (Figure 3.20 and 3.21). The minimum turning radius of an aircraft is

$$
R_{\text {min }}^{T}=\frac{V_{a}^{2}}{g \sqrt{n^{2}-1}}
$$

and the maximum turning rate is

$$
\omega_{\max }^{T}=\frac{g \sqrt{n^{2}-1}}{V_{a}}
$$




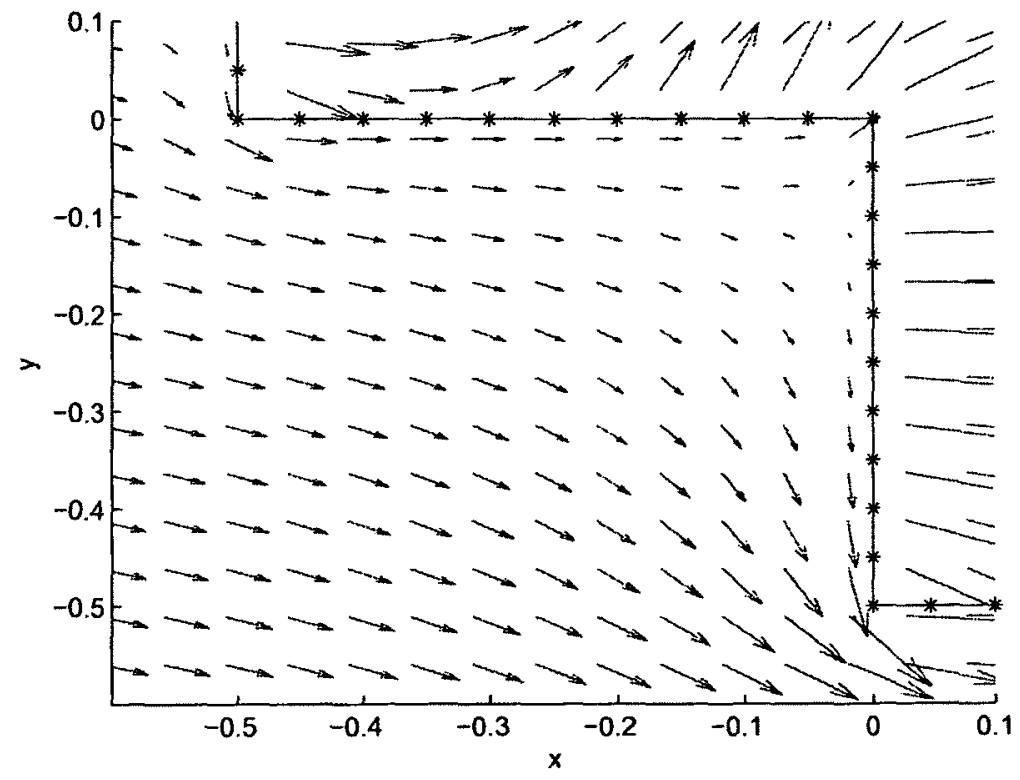

Figure 3.19: Concave region of a concave obstacle with enhanced accuracy

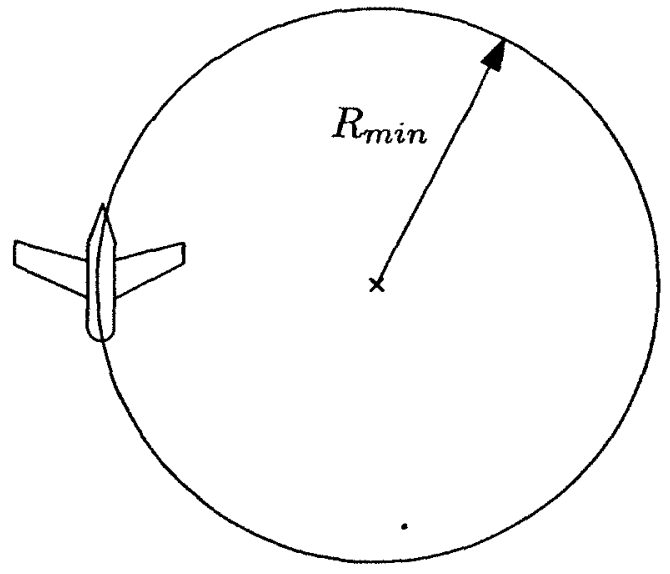

Figure 3.20: Top view of an aircraft performing the tightest turn possible

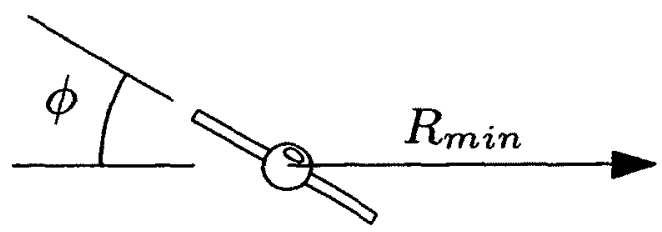

Figure 3.21: Front view of an aircraft performing the tightest turn possible ( $\phi$ is the bank angle) 
where $V_{a}$ is the aircraft's airspeed, $g=9.81 \mathrm{~m} / \mathrm{s}^{2}$ is the Earth's gravitational acceleration, and $n$ is the load factor of the aircraft defined as

$$
n=\frac{L}{W}
$$

where $L$ and $W$ are the aircraft's lift and weight, respectively. In turning flight, it can be shown that

$$
n=\frac{1}{\cos \phi}
$$

where $\phi$ is the aircraft's bank angle. From (3.59) in order to get the tightest turn at a given speed the highest load factor should be used. From (3.61)

$$
n_{\max }=\frac{L_{\max }}{W}=\frac{1}{2} \rho V_{a}^{2} \frac{C_{L, \max }}{W / S}
$$

where $\rho$ is the density of air, $S$ is the aircraft's wing surface and $C_{L, \max }$ is the maximum lift coefficient of the wings. Using (3.63) and (3.62) the bank angle required for the tightest turn possible can be calculated.

Similarly, the aircraft has a limitation on the tightest pull-up turn it can make (Figure 3.22).

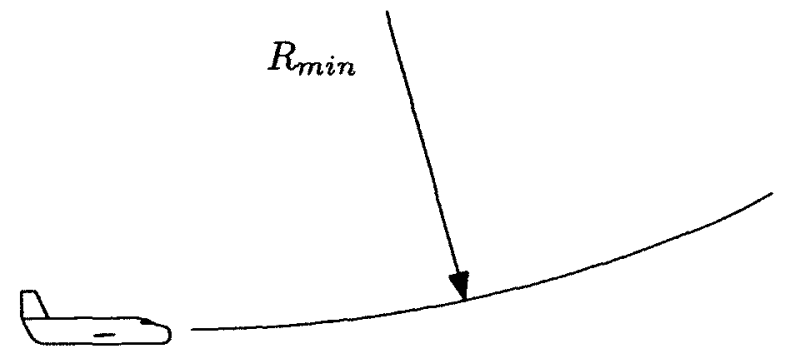

Figure 3.22: Side view of an aircraft performing the tightest pull-up turn possible

The minimum pull-up radius and maximum pull up rate can be calculated from

$$
R_{\min }^{P}=\frac{V_{a}^{2}}{g(n-1)}
$$




$$
\omega_{\max }^{P}=\frac{g(n-1)}{V_{a}},
$$

where $n_{\max }$ can be obtained from (3.63).

It should be noted that the constraints on turning and pull-up radii introduced in this section are for steady flight, and are therefore somewhat conservative. In an emergency, tighter turns could likely be executed for short periods of time (a scenario that would arise if the obstacle was detected late).

\subsubsection{Integration of $\mathrm{OA}$ and Aircraft Constraints}

In this section, a method is developed to incorporate the aircraft constraints introduced in Section 3.5.2.1 into the panel method.

Recall from Section 3.5.1 that obstacles are initially modelled as squares. As illustrated in Figure 3.23, the pathlines close to the obstacle centerline can have quite radii of curvature. In order to decrease the curvature of the flow's pathlines, the flow is "directed" by adding wedges to the front and back of obstacles. These wedges make the pathlines separate from their original course sooner than if the wedges were not present. This results in pathlines having larger minimum radii of curvature. The difference between the curvature of the pathlines can be seen by comparing Figures 3.23 and 3.24 , which is most noticeable for pathlines close to the centerline of the obstacle $(y=0) .^{9}$

To correlate the minimum turning/pull-up radius to the wedge length (Figure 3.25$)$, the minimum radius of curvature $\left(R_{\min }\right)$ was computed along all pathlines for different wedge lengths $(h)$.

\footnotetext{
${ }^{9}$ Note that for this type of polygon shape the use of sub-panels is not required and with extensive testing it was seen that even without sub-panels flow never enters the obstacles.
} 


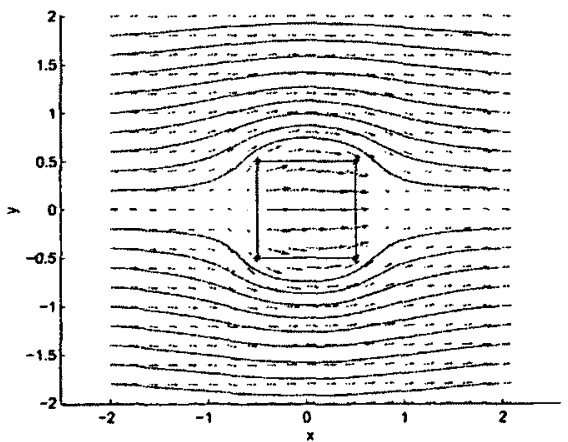

Figure 3.23: Simple unit length square

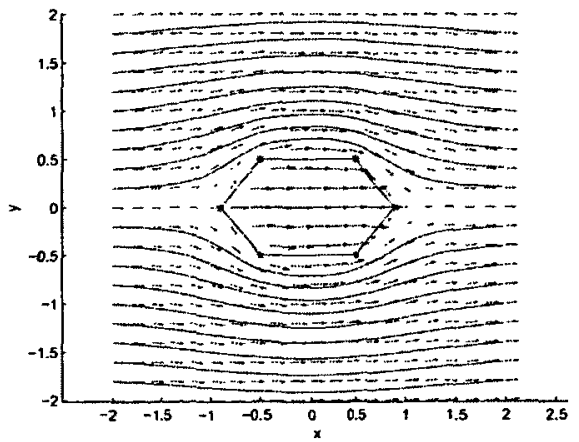

Figure 3.24: Square with wedges added

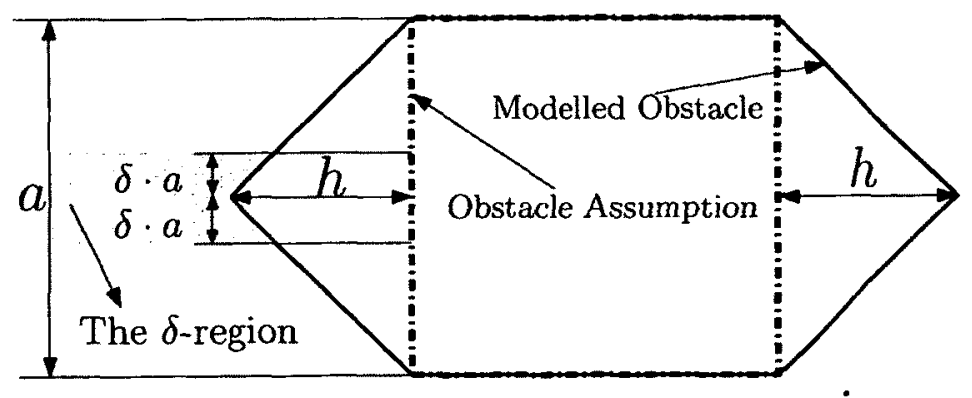

Figure 3.25: Modelled obstacle 
From figure 3.26 , for any two adjacent points ${ }^{10}$ on a pathline

$$
R=\frac{d}{2 \sin \theta / 2} \quad, \quad \theta=\cos ^{-1} \frac{\overrightarrow{V_{1}} \cdot \vec{V}_{2}}{\left|\overrightarrow{V_{1}}\right| \cdot\left|\vec{V}_{2}\right|}
$$

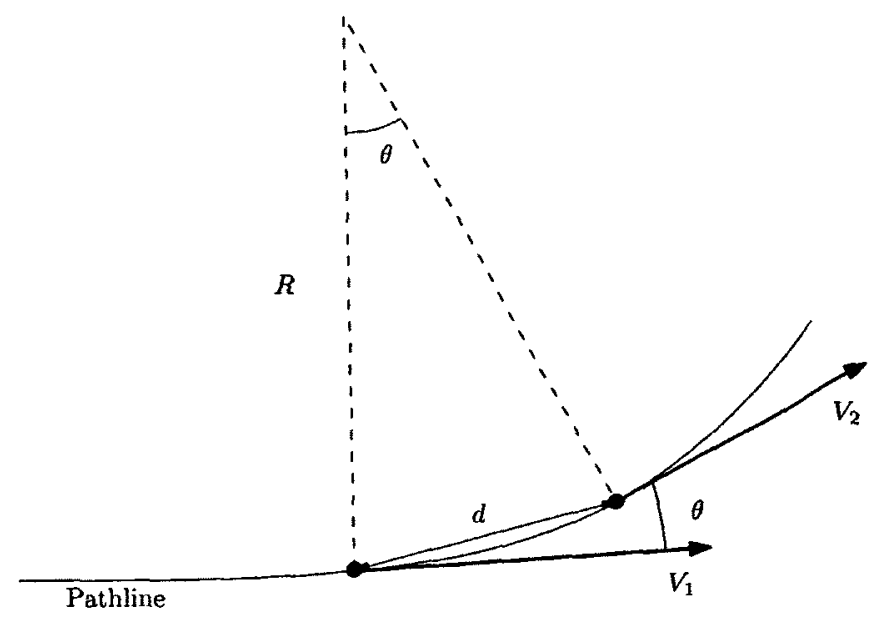

Figure 3.26: Computing approximate radius of curvature using two adjacent points

Figure 3.27, shows the minimum radius of curvature for three pathlines. It can be noticed that the closer the pathlines are to the obstacle's horizontal axis of symmetry, the lower $R_{\min }$ is.

While computing $R_{\min }$ for the pathlines of different wedge lengths, the pathlines whose starting points were within $[-\delta, \delta] a$ (the " $\delta$-region") were skipped. As a result, for a given obstacle and given $R_{\text {min }}$, a shorter wedge length would be required.

In Figure 3.28, $R_{\min }$ was calculated for different wedge lengths where $\delta=0.1$ was selected ${ }^{11}$. When an obstacle is encountered, this graph is used to determine the wedge length required to satisfy the aircraft's minimum turning/pull-up radius. Further explanation on how to use this graph for OA is given in Section 5.1.4.1

\footnotetext{
${ }^{10}$ The pathlines were plotted using the stream2 function in Matlab. This function returns an array of vertices that are used to plot the pathlines. The points referred to here are these vertices.

${ }^{11}$ The reader is referred to Appendix $A$ for the discussion on why $\delta=0.1$ was selected.
} 


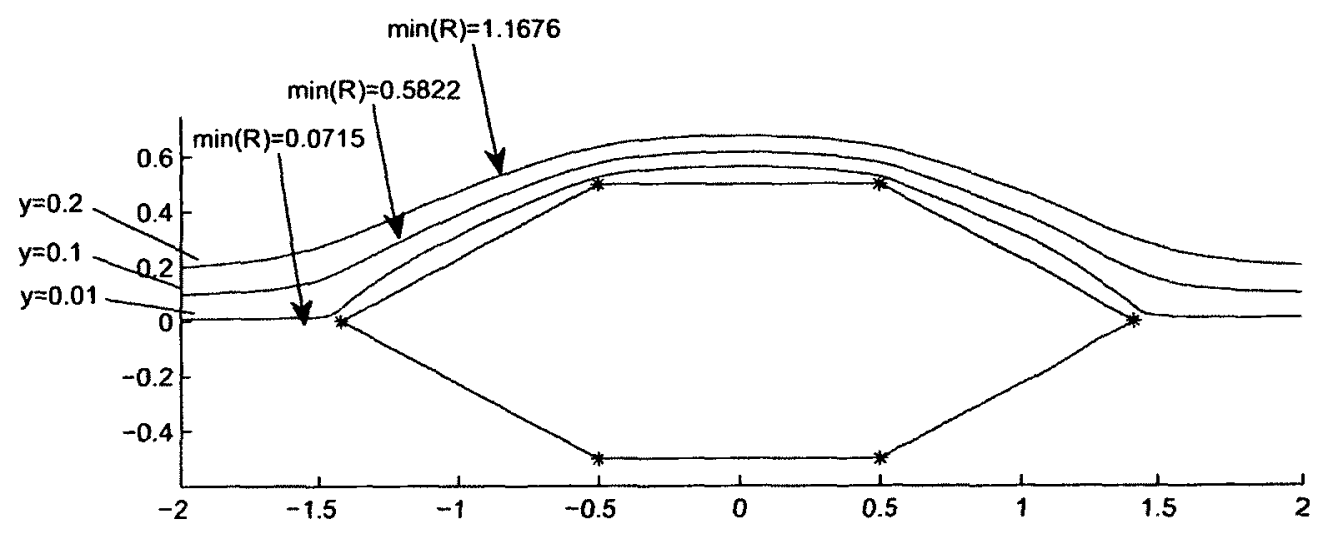

Figure 3.27: Three pathlines in close proximity of the horizontal axis of symmetry

It should be noted that the $R_{m i n}-h$ graph was initially computed for a unit width square. In Appendix B, it is shown that if the obstacle is scaled by a factor of $a$, the pathlines' shapes do not change and are scaled by $a$ as well. Consequently, $R_{\min }$ is also scaled by $a$. Therefore, to generalize the $R_{\min }-h$ graph for obstacles of any width, $R_{\min }$ and $h$ were substituted by $R_{\min } / a$ and $h / a$, respectively where $a$ is obstacle width.

If the aircraft's starting position happens to be within the $\delta$-region of the obstacle's centerline, such as in Figure 3.29, the obstacle's width, $a$, is increased to $a_{n e w}$, so that the aircraft would be outside of the $\delta$-region of the bigger "pseudo-obstacle". Note that the obstacle's depth remains unchanged. From Figure 3.29, it can be seen that

$$
a_{n e w}=\frac{a}{1-2 \delta} \text {, }
$$




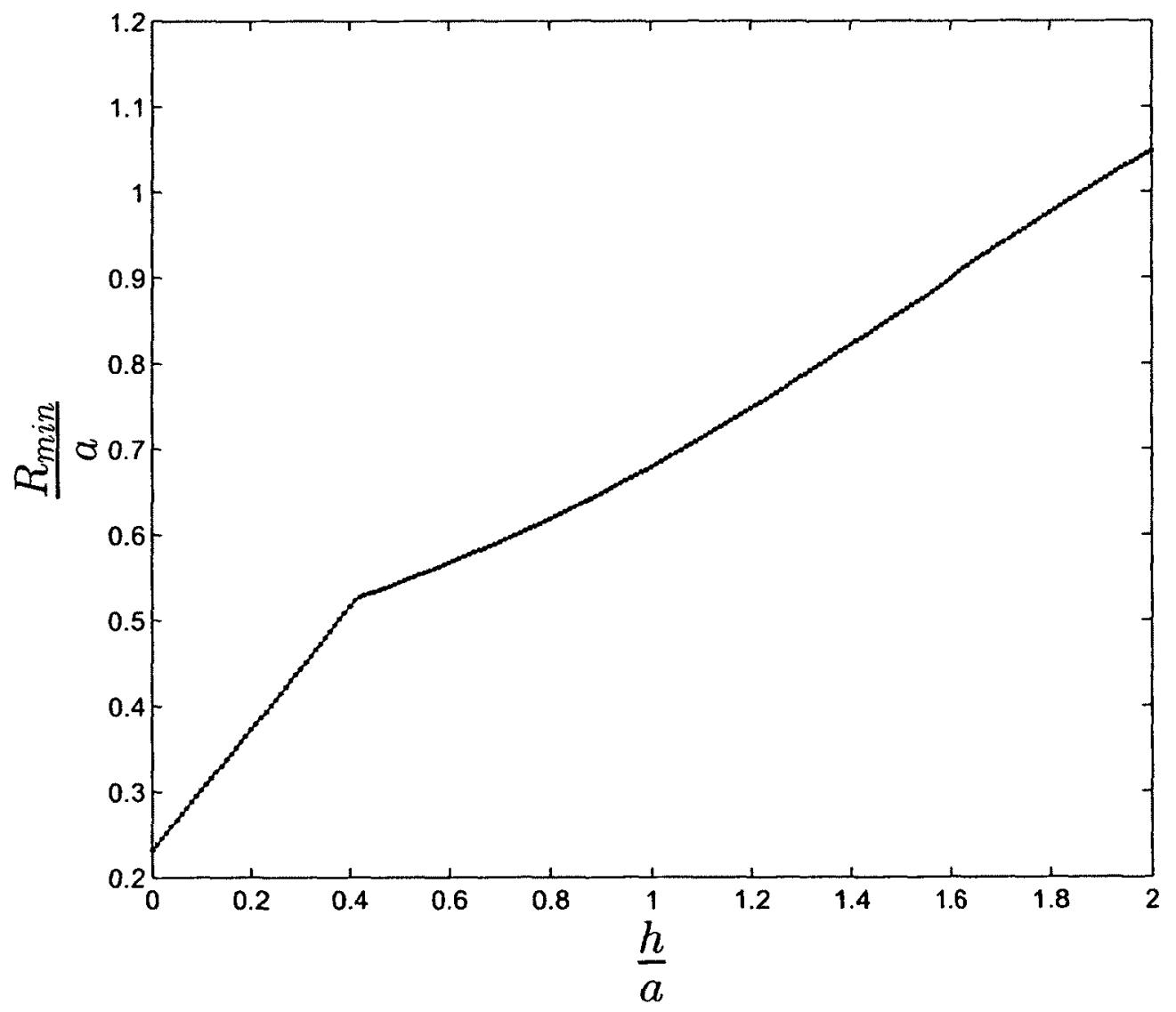

Figure 3.28: Minimum radius of curvature for different wedge lengths. Pathlines with starting points within $[-0.1,0.1] a$ of the centerline were excluded. 


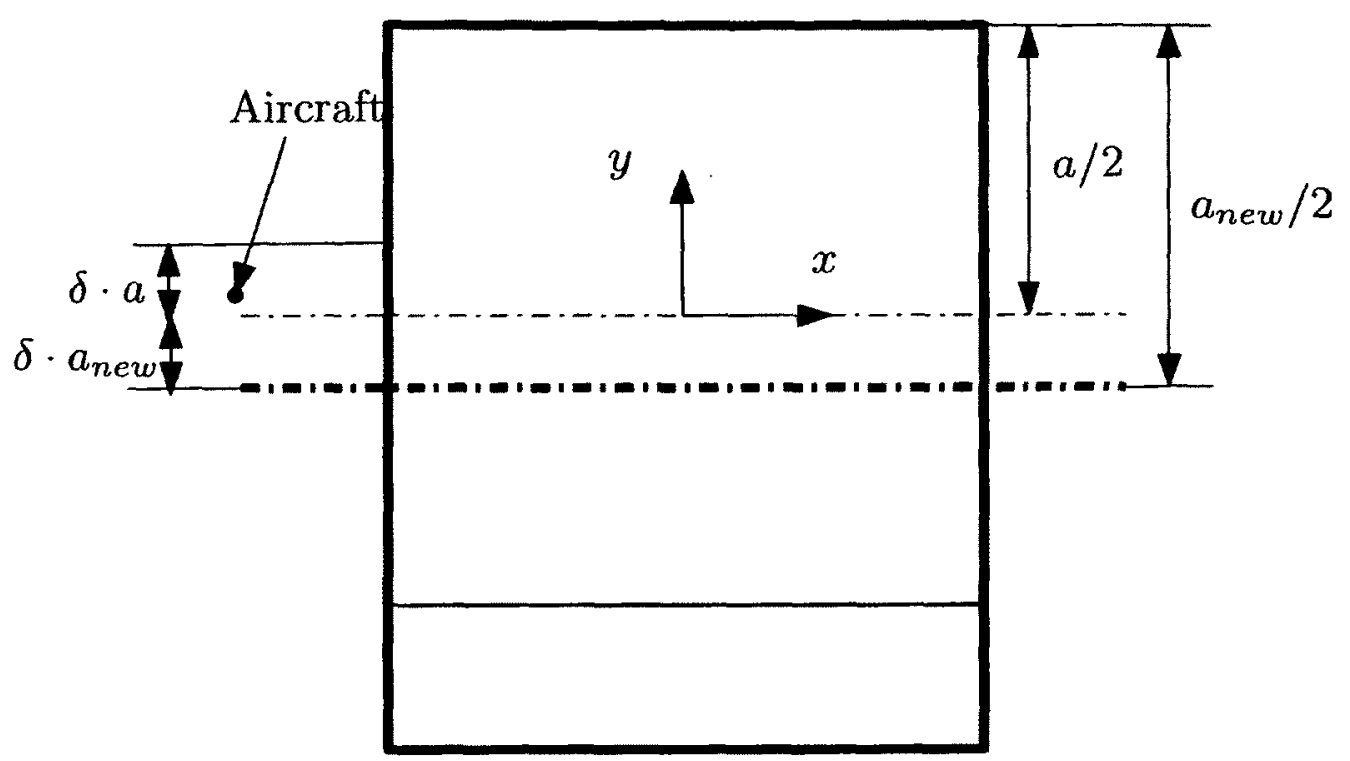

Figure 3.29: Increasing obstacle size to avoid pathlines with tight turns

where for $\delta=0.1,(3.67)$ changes to

$$
a_{\text {new }}=1.25 a
$$

\subsubsection{Multiple Obstacles}

The panel method introduced in Section 3.4 was derived for a single closed obstacle. However, its usage can be extended to multiple obstacles. This is done by extending the enumeration of panels to the additional obstacles such as in Figure 3.30. However, in Section 5.1.1, it will be seen that if the obstacles are so close that the aircraft can't safely fly in between them, the obstacles are fit with a bigger polygon, and will be modeled as a single large obstacle. In Figure 3.31, the pathlines of two obstacles that are immersed in a uniform flow are plotted. 


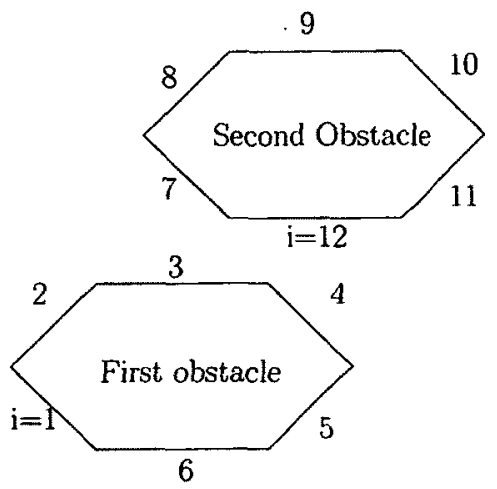

Figure 3.30: Enumeration of panels for multiple obstacles

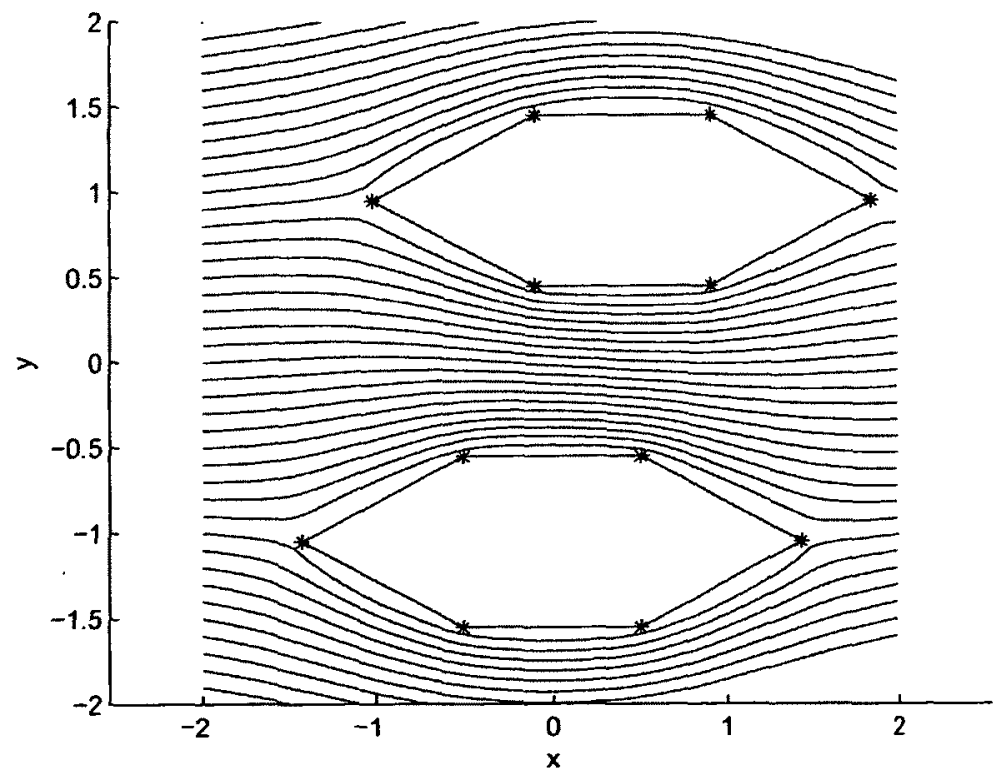

Figure 3.31: Two obstacles of unit length with wedges $h=0.92(a=1)$ 


\subsubsection{Adding a Sink}

Until here, in order to compute a velocity field that avoids obstacles; they were immersed in a uniform flow. For a single obstacle, the pathlines were seen to be symmetric with respect to the horizontal centerline of the modelled obstacle and the computed velocity field eventually guided the aircraft back to its original flight path. However, if multiple obstacles are present the pathlines are not symmetric (as illustrated in Figure 3.31) and the aircraft would not return to its nominal flight path after avoiding the obstacles. Moreover, due to autopilot lag and wind the aircraft will not exactly follow the pathlines it starts on. By adding a sink on the nominal flight path, pathlines will be directed back to the nominal flight path. The added sink can be thought as a goal point the aircraft wants to reach. In this section, the panel method is derived in the presence of a sink ${ }^{12}$.

From (3.26), the added sink's potential field is

$$
\Phi_{g}=\frac{\Lambda_{g}}{2 \pi} \ln r_{g}
$$

where

$$
r_{g}=\sqrt{\left(x-x_{g}\right)^{2}+\left(y-y_{g}\right)^{2}}
$$

and $\left(x_{g}, y_{g}\right)$ is the location of the sink, and $\Lambda_{g}<0$ is the sink strength.

The velocity field induced by the added sink is

$$
\vec{V}_{g}=\nabla \Phi_{g}=\frac{\Lambda_{g}}{2 \pi}\left(\frac{x-x_{g}}{r_{g}^{2}}, \frac{y-y_{g}}{r_{g}^{2}}\right)
$$

\footnotetext{
${ }^{12}$ Selecting the location of the sink is covered in Section 5.1.4 and selecting the sink strength is covered in Sections 3.5.4.1 and 5.1.4.
} 
The addition of the sink changes (3.33) to

$$
V_{\infty\left(n_{i}\right)}+V_{\left(n_{i}\right)}+V_{g_{\left(n_{i}\right)}}=0
$$

where

$$
V_{g_{\left(n_{i}\right)}}=V_{g} \cdot \hat{n}_{i}
$$

Therefore (3.42) changes to

$$
[I][\vec{\lambda}]=\left[-\vec{V}_{\infty_{n}}\right]+\left[-\vec{V}_{g_{n}}\right]
$$

where

$$
\left[-\vec{V}_{g_{n}}\right]=\left[\begin{array}{c}
\vdots \\
-V_{g} \cdot \hat{n}_{i} \\
\vdots
\end{array}\right]
$$

Furthermore (3.43) changes to

$$
\Phi_{T}=\Phi_{u}+\Phi_{\circ}+\Phi_{g}
$$

Therefore

$$
V_{x}(x, y)=V_{\infty}+\sum_{j=1}^{n} \frac{\lambda_{j}}{2 \pi} J_{x, j}+\frac{\Lambda_{g}}{2 \pi}\left(\frac{x-x_{g}}{r_{g}^{2}}\right)
$$

and

$$
V_{y}(x, y)=\sum_{j=1}^{n} \frac{\lambda_{j}}{2 \pi} J_{y, j}+\frac{\Lambda_{g}}{2 \pi}\left(\frac{y-y_{g}}{r_{g}^{2}}\right)
$$

where $J_{x, j}$ and $J_{y, j}$ are still calculated from (3.48) and (3.49).

In Figure 3.32, a sink is added at $(1.5,0)$ to the unit square of Figure 3.11.

By comparing Figure 3.32 to 3.11 , it can be seen that the sink mainly affects the 


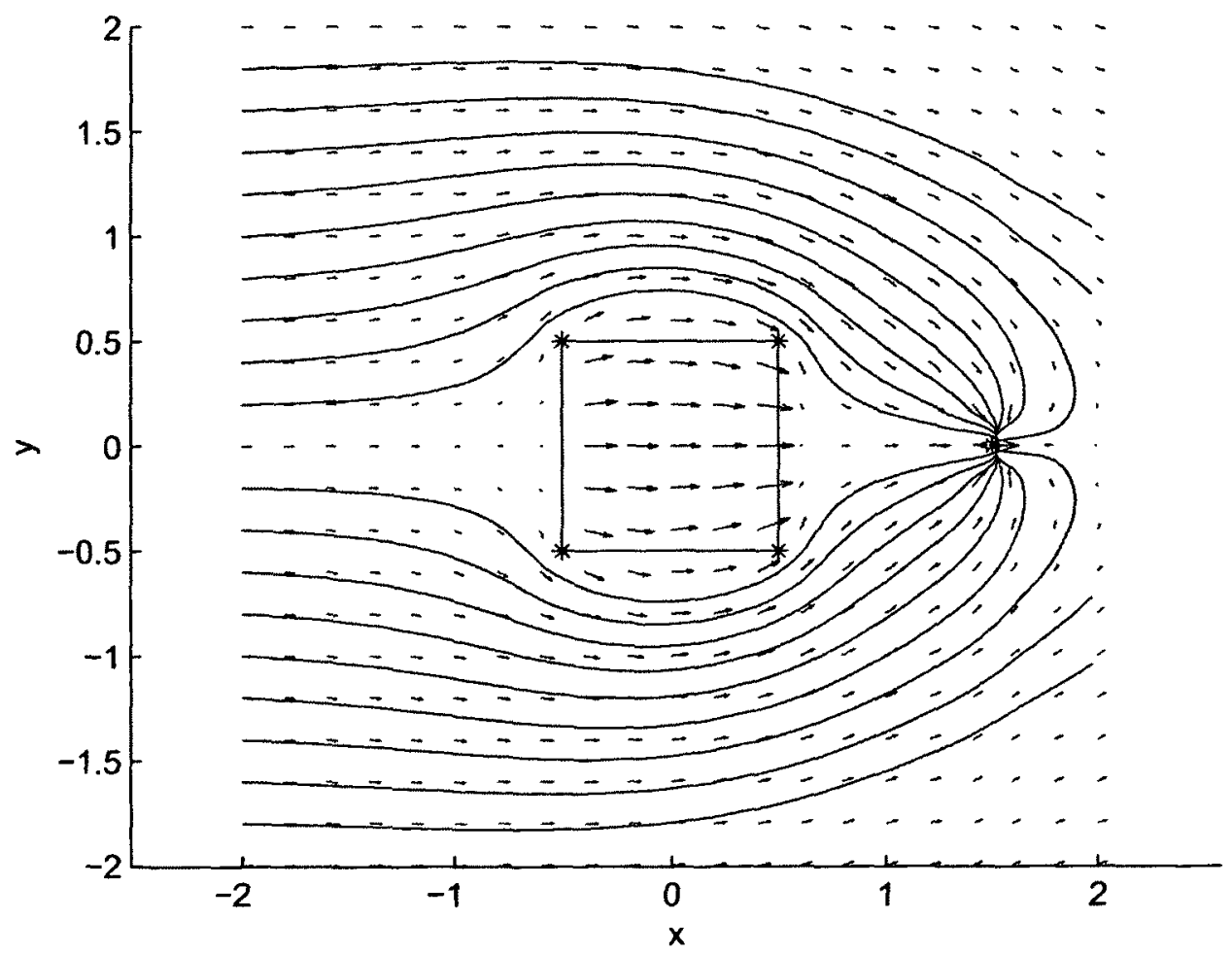

Figure 3.32: Velocity field flow pathlines around a unit square.

$$
V_{\infty}=1 m / s, \Lambda_{g}=-3 m^{2} / s
$$


pathlines after they have avoided the obstacle. Therefore, even if in this region the pathlines would have radii of curvature that would be infeasible for the aircraft to follow, it would merely mean that the aircraft would reach its nominal flight path with some delay.

\subsubsection{Computing the Required Sink Strength}

The sink strength, $\Lambda_{g}$, defines the amount of flow that will be sinked. A uniform horizontal flow with $V_{\infty}$ and a sink are considered. The amount of flow required to be sunk is assumed to have a cross section of $l$, i.e. $y=\left[-\frac{l}{2}, \frac{l}{2}\right]$. The sink strength required to absorb this amount of flow is

$$
\Lambda_{g}=-V_{\infty} \cdot l \quad m^{2} / s
$$

In Figure 3.33, a uniform flow with $V_{\infty}=1 \mathrm{~m} / \mathrm{s}$ and a sink of strength $\Lambda_{g}=-2 \mathrm{~m}^{2} / \mathrm{s}$ is used. The dashed line specifies the boundary pathline of flow being sunk. It can be seen that all pathlines starting within $y=\left[-\frac{l}{2}, \frac{l}{2}\right]$ are successfully absorbed by the $\operatorname{sink}^{13}$

Note that further integration of potential flow into OA is covered in Chapter 5

\subsection{Summary}

In this chapter, the usage of potential flow and the panel method for OA were introduced and initial results for OA were presented. Furthermore, the computation time and accuracy of the panel method were discussed. Finally, obstacle modelling was covered and a new approach to obstacle modeling was introduced that takes aircraft constraints into consideration for potential flow OA.

\footnotetext{
${ }^{13}$ Selecting $l$ is covered in Section 5.1.4.
} 


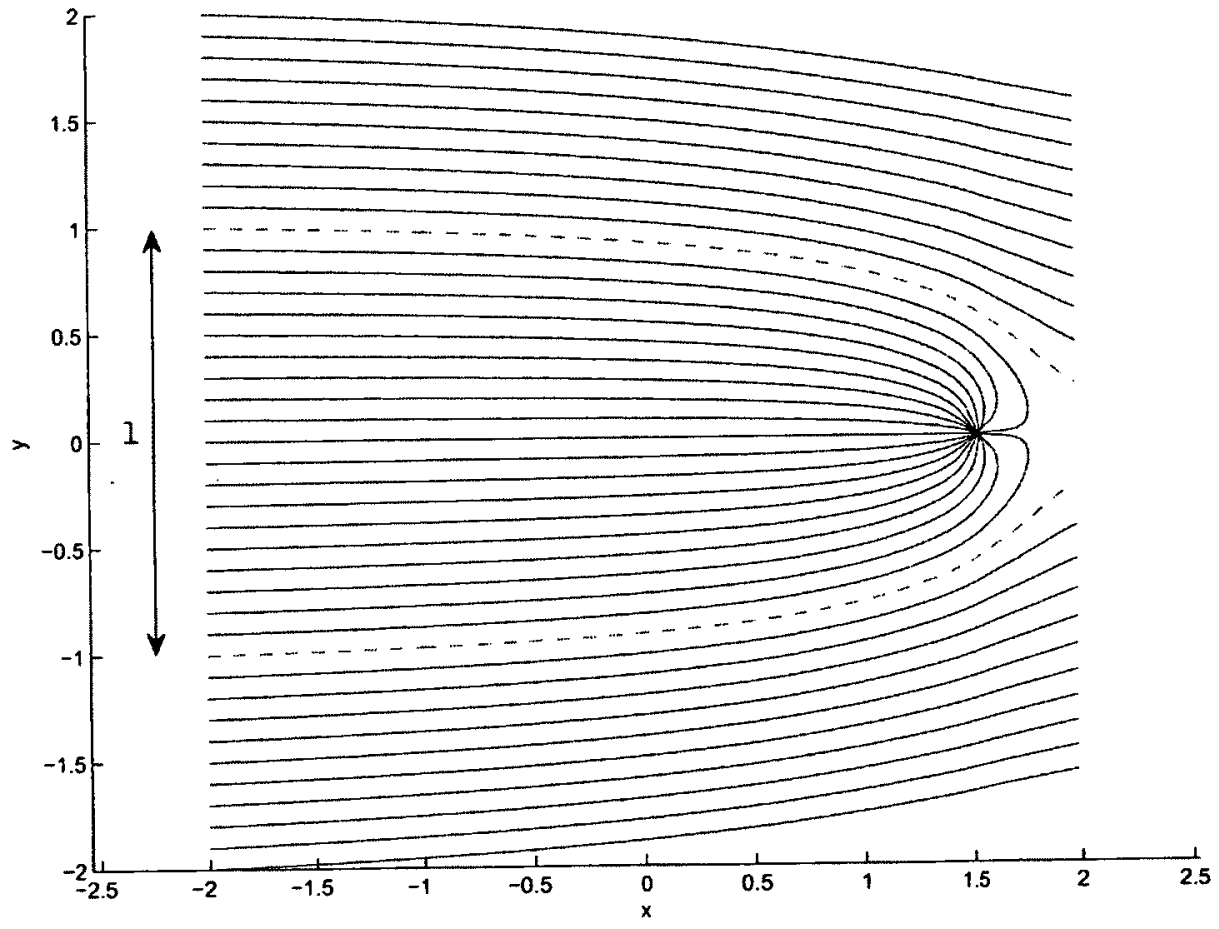

Figure 3.33: A uniform flow and a sink. $V_{\infty}=1 \mathrm{~m} / \mathrm{s}, l=2 m$ and $\Lambda=-2 \mathrm{~m}^{2} / \mathrm{s}$ 


\section{Chapter 4}

\section{Aircraft Autonomous Flight \& Simulation Framework}

The prerequisite to implementing $\mathrm{OA}$ on an aircraft is for that aircraft to be able to operate autonomously under obstacle-free circumstances. Therefore, in this chapter, a typical autonomous flight architecture is introduced. Then, to incorporate OA in the flight architecture, some modifications are made to it. It should be noted that the development of such an architecture is secondary to the main objective of this thesis, which is OA. Furthermore, a simulation framework that OA algorithms can be tested on is introduced. Finally, the performance of the modified autonomous flight architecture is assessed.

\subsection{Simulation Framework}

In order to implement the OA method developed in Chapter 3, an aircraft simulation framework is required. In this research, the AeroSim aeronautical simulation blockset, • developed by Unmanned Dynamics ${ }^{\circledR}$, is used.

The AeroSim blockset can be used to create a non-linear six degree of freedom 
aircraft model that inputs aircraft controls and simulates its motion and sensor outputs within Simulink ${ }^{\circledR}$. In the blockset, a model of the Aersonde UAV is offered. For OA testing, the Aerosonde UAV model is used.

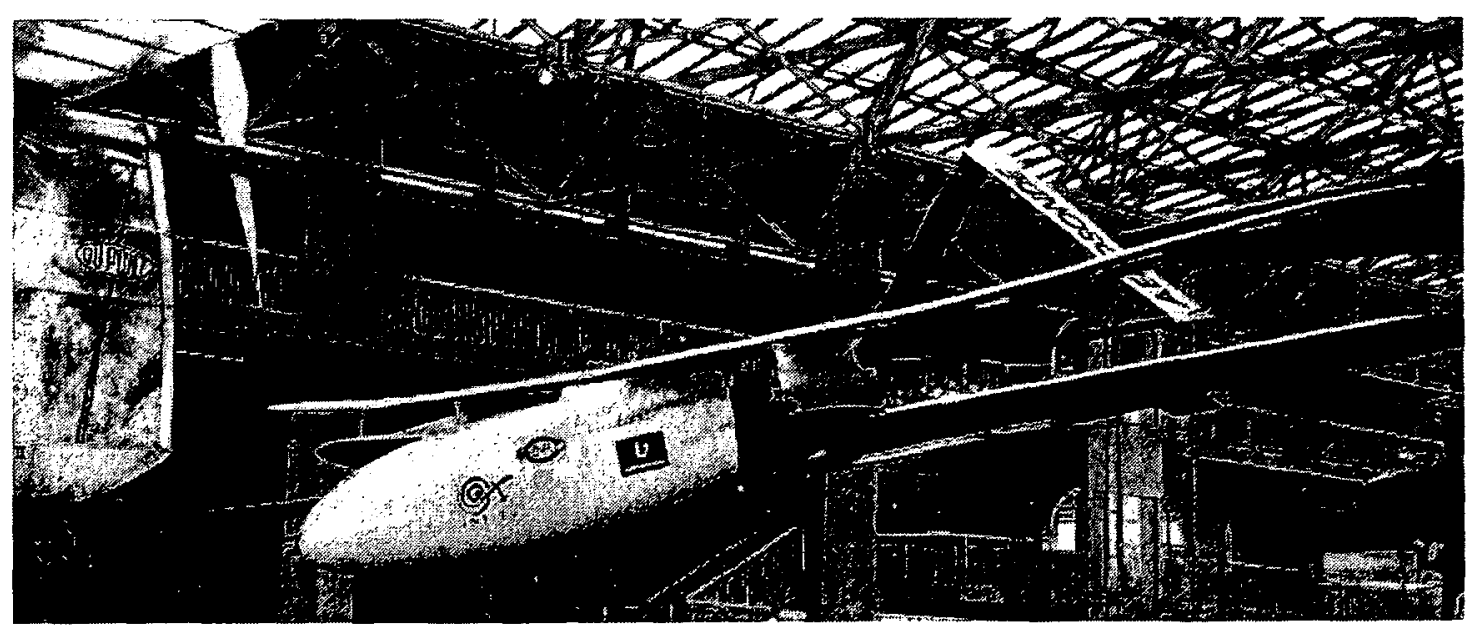

Figure 4.1: The Aerosonde UAV model at the museum of flight in Seattle

The Aerosonde UAV, shown in Figure 4.1 [48], is a small UAV developed for weather-reconnaissance and remote-sensing missions, and it was the first UAV to cross the north-Atlantic in $1998[48,49]$. A summary of the aircraft's specifications are listed in Table $4.1[48,50]$.

For visualization of the aircraft's response in three dimensions, FlightGear, which is an open-source flight simulator, is used ${ }^{1}$. Note that the equations of motion are solved in Simulink, and FlightGear is only used for visualization. In Figure 4.2, the Simulink model and the FlightGear visualization of the Aerosonde model is illustrated.

\subsection{Autonomous Flight Architecture}

In order for an aircraft to fly autonomously, several steps need to be taken. Firstly, the mission is broken down to flight commands by a mission planner. Secondly, the

\footnotetext{
${ }^{1}$ In this thesis FlightGear v0.9.8 was used.
} 

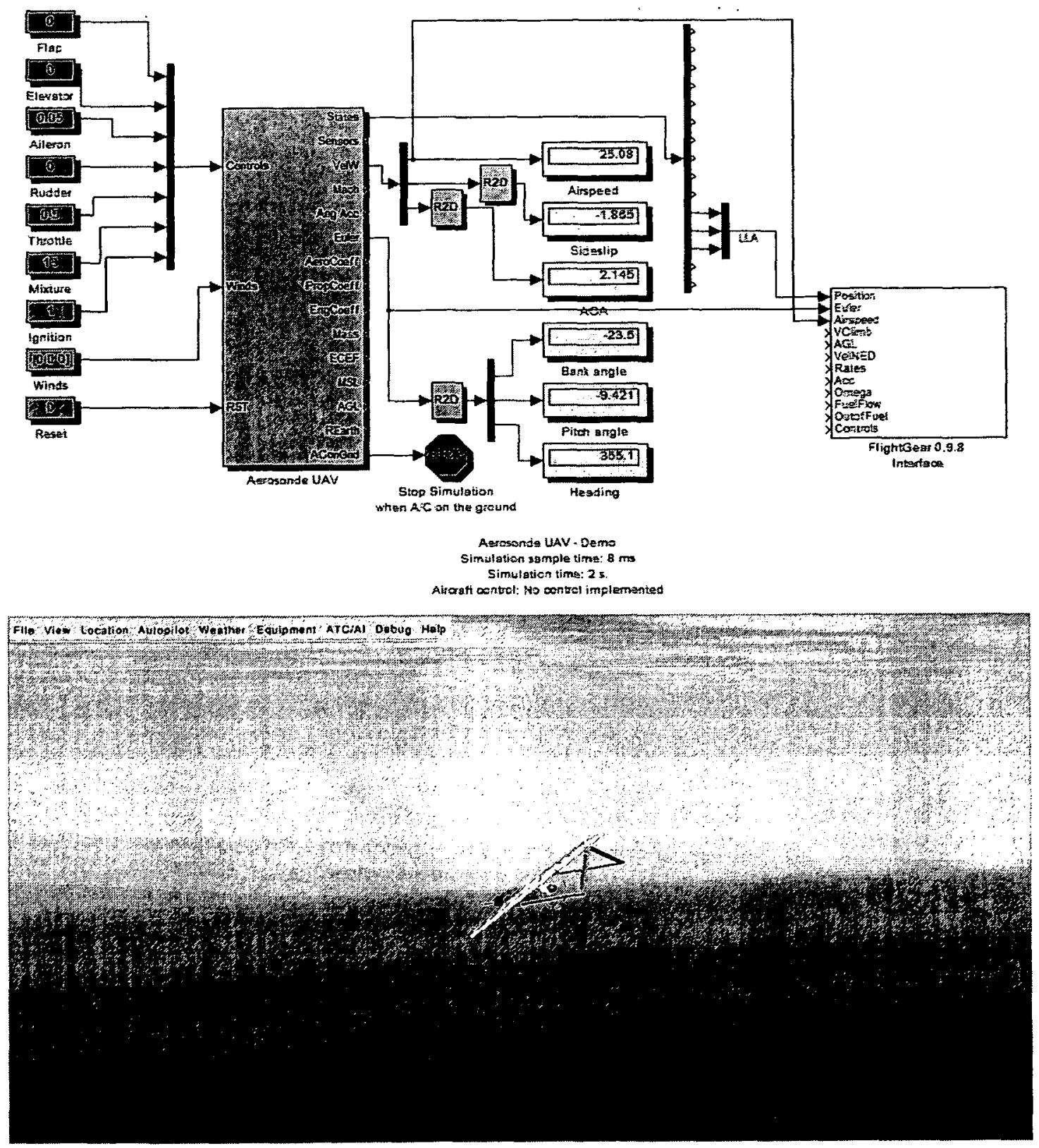

Figure 4.2: Aerosonde Simulink model and FlightGear visualization for a $0.05 \mathrm{rad}$ aileron deflection 


\begin{tabular}{|c|c|}
\hline Weight & $27-30 \mathrm{lb}$ \\
\hline Length & $6 \mathrm{ft}$ \\
\hline Height & $2 \mathrm{ft}$ \\
\hline Wing span & $10 \mathrm{ft}$ \\
\hline Wing Area & $6 \mathrm{ft}^{2}$ \\
\hline Engine & $24 \mathrm{cc}, 1.2 \mathrm{~kW}$ \\
\hline Cruise Speed & $51 \mathrm{mph}$ \\
\hline Range & $2044 \mathrm{miles}$ \\
\hline Altitude range & Up to $20000 \mathrm{ft}$ \\
\hline Payload & Maximum $5 \mathrm{lb}$ with full fuel \\
\hline
\end{tabular}

Table 4.1: Aerosonde UAV specifications.

flight commands are converted to actuator commands that control the aircraft, which is done by an autopilot. This process is summarized in Figure 4.3.

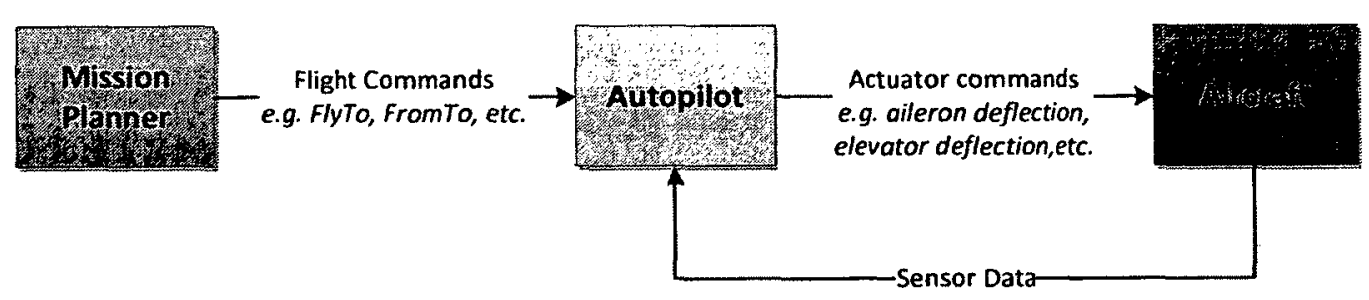

Figure 4.3: Autonomous flight architecture

Typically, flight commands define how an aircraft flies from one point to another. These points are called waypoints (WP). An example of a flight command is a "FromTo" command, where the aircraft is commanded to fly from an initial WP to a secondary one, while maintaining the course between the two WPs. Another example is a "FlyTo" command, where the aircraft flies from one WP to another, while its course is not constrained. Therefore this command can be used to perform a turn. 
In Figure 4.4, a mission consisting of From To and FlyTo commands is illustrated ${ }^{2}$.

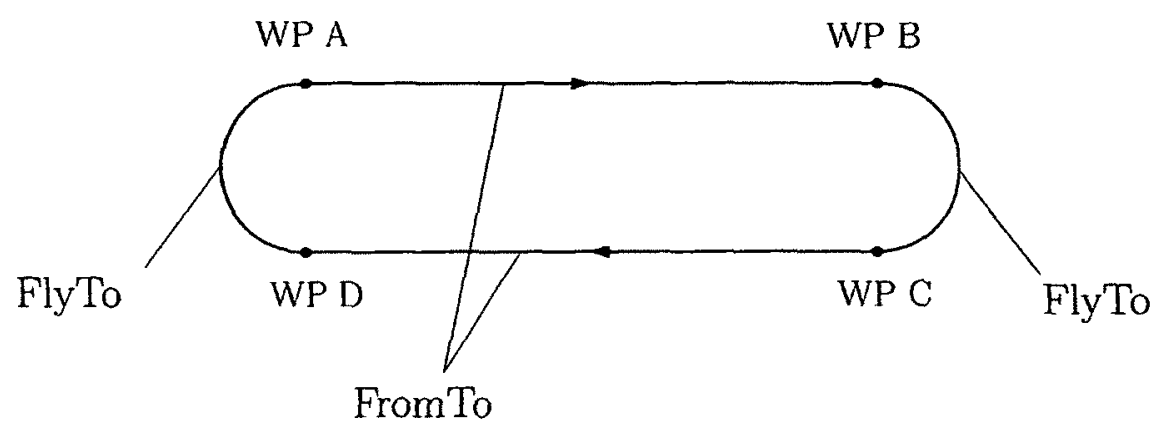

Figure 4.4: A mission consisting of FlyTo and FromTo commands

\subsubsection{Autopilot Architecture}

The autopilot architecture described in this section is the architecture used in the commercial off-the-shelf autopilot manufactured by MicroPilot ${ }^{\circledR}$ [51]. This architecture is used to maintain similarity between the simulations and the MicroPilot hardware used on the UAV test bed that is described in Section 6.1.2.

The autopilot has a high and low-level controller. The high-level controller computes parameters such as required heading, altitude, etc. based on the flight commands defined by the mission planner. The low-level controller then computes the actuator inputs required to perform the flight commands. The autopilot architecture is illustrated in Figure 4.5. The control laws used in Figure 4.5 are PID controllers, which is the type of controller that most commercial autopilots use [52]. ${ }^{3}$

The high-level controller output depends on the flight command dictated by the mission planner. For example, when From To is commanded between two waypoints, a heading command is computed using the controller illustrated in Figure 4.6 where the crosstrack error is defined in Figure 4.7. The controller computes heading commands

\footnotetext{
${ }^{2}$ From To and FlyTo are names used by MicroPilot.

${ }^{3}$ Examples of other autopilot architectures developed for UAVs are fuzzy based autopilots $[50,53]$ and neural-network based autopilots [54].
} 


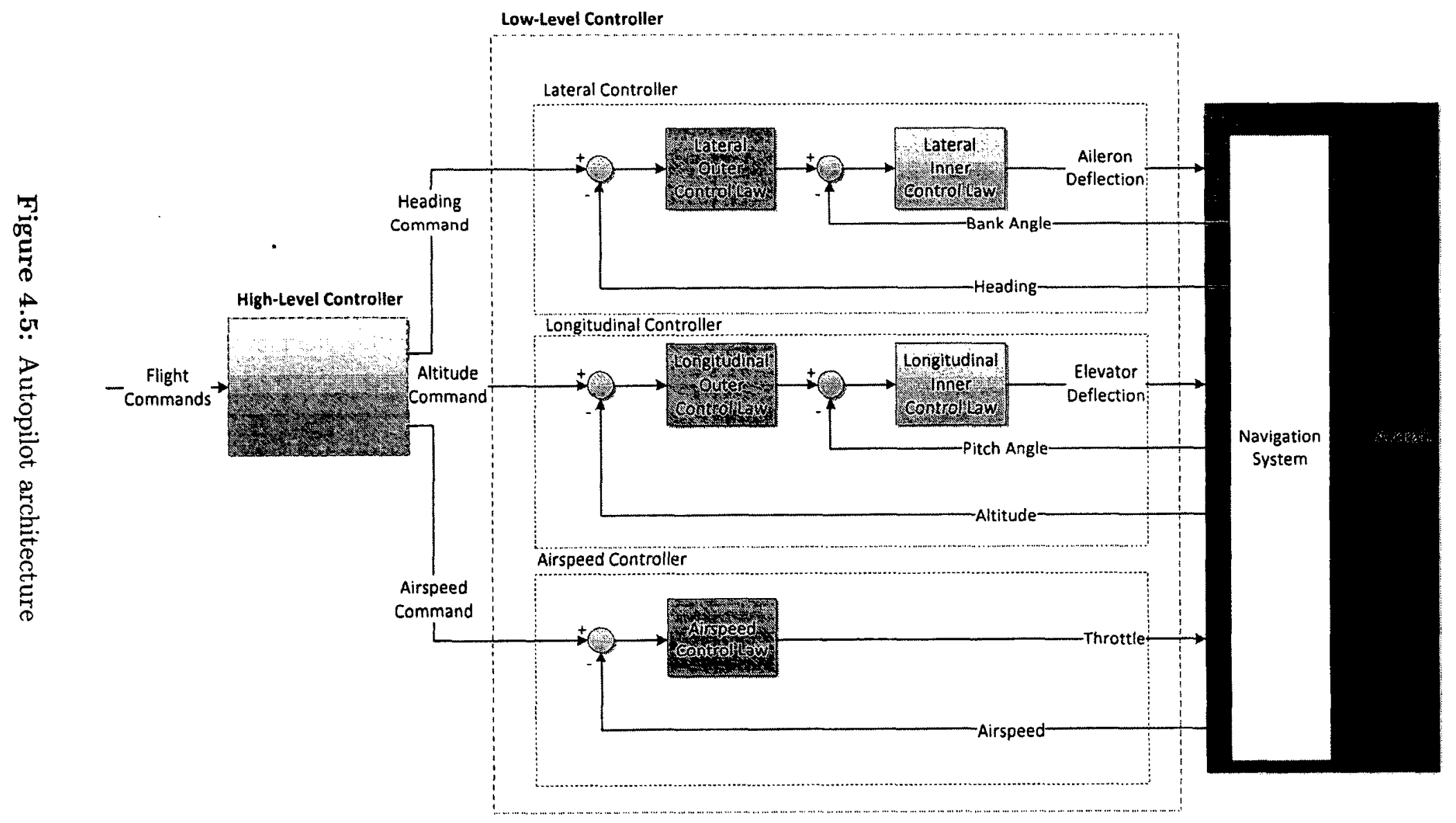


that reduce the crosstrack error while flying to the next waypoint, hence the aircraft is being commanded to return to its intended course.

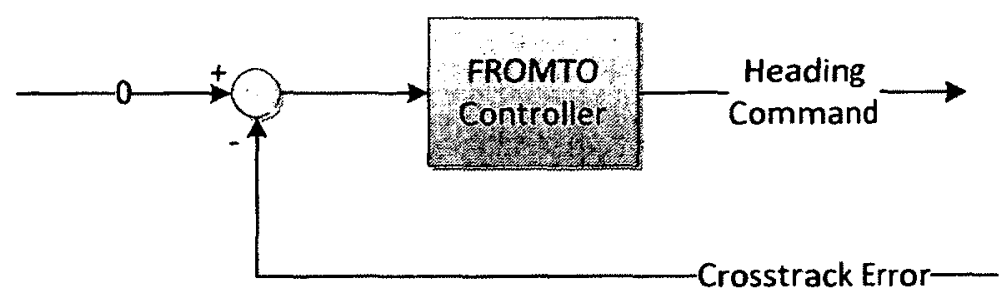

Figure 4.6: Heading from crosstrack error controller

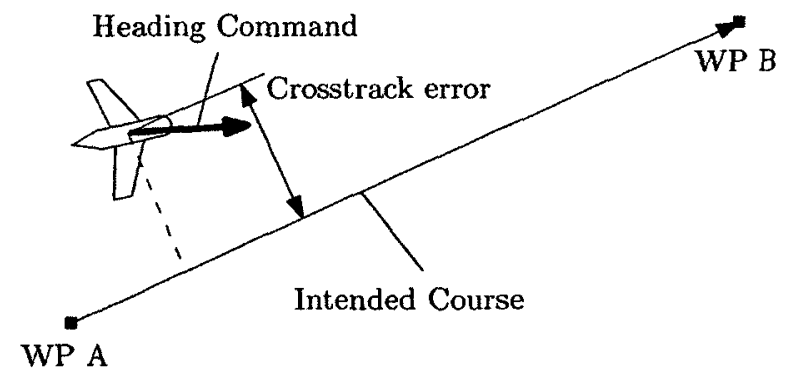

Figure 4.7: Definition of crosstrack error that is used in a FromTo controller

Furthermore, when Fly To is commanded, the heading command is calculated such that it always points toward the next waypoint. For more complex flight commands the reader is referred to [51].

The low-level controller consists of three controllers: lateral, longitudinal and an airspeed controller. The lateral and longitudinal controllers each consist of an inner and outer control loop.

In the lateral controller, the outer control loop is employed to determine the required bank angle from the error in heading. The inner control loop then computes aileron deflection from the bank angle error. Similarly, in the longitudinal controller, the required pitch angle is computed from the altitude error in the outer control loop. Then, the required aileron deflection is computed from the pitch angle error 
in the inner loop. Finally, the airspeed is directly controlled by setting the throttle command based on the airspeed error.

Note that depending on the flight command, the low-level controller might slightly change from that of Figure 4.5. For instance, in a descent maneuver, the longitudinal outer controller loop calculates the required pitch angle from the error in the desired descent rate.

The low-level controller can be expanded with additional controllers for higher performance. For instance, the MicroPilot autopilot has an additional controller that calculates rudder deflection from lateral acceleration error ${ }^{4}$. However, the three controllers presented here are the crucial controllers required to guide the aircraft. It should be noted that in the controllers above, control surface deflection commands are limited to prevent impossible deflections. Furthermore, the roll and pitch angle commands are also limited to prevent the aircraft from rolling over and stalling, respectively ${ }^{5}$.

All control laws in Figure 4.5 are SISO PID controllers. Therefore, the coupling of the controlled parameters has not been considered [55]. To decrease the effect of this shortcoming, feed-forwards are used in several control laws. For instance, a feed-forward term taken from the absolute value of bank angle is added to the inner longitudinal control law to increase elevator deflection while banking to compensate for the loss of lift and to maintain the aircraft's altitude [51].

\footnotetext{
${ }^{4}$ This control loop is used to coordinate turns and prevent sideslip. However, its usage is not necessary for aircraft guidance.

${ }^{5}$ For restrictions on maximum pitch angle and rate of climb, the reader is referred to [40].
} 


\subsection{Autonomous Flight Architecture with Obsta- cle Avoidance}

The architecture described in Section 4.2 needs to be modified to be able to incorporate an OA scheme. Firstly, a decision-making component needs to be added to decide when the flight commands (generated by the mission planner) are controlling the UAV and when the OA algorithm is. This subsystem, called Selector, decides based on the aircraft's location and data from obstacle detection. Therefore the architecture of Figure 4.3 changes to that of Figure 4.8. In Section 5.1.3, the Selector's decision-making process is explained.

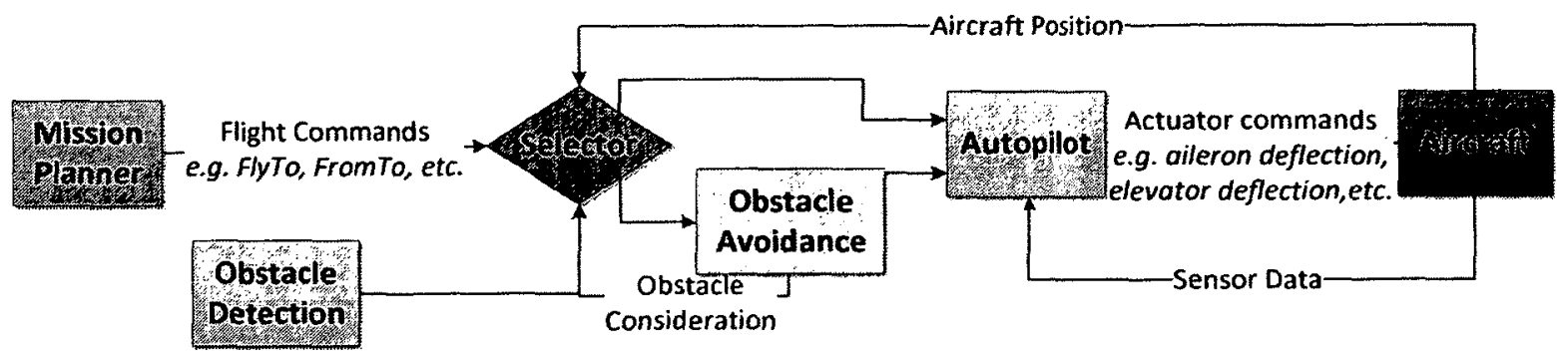

Figure 4.8: Autonomous flight architecture with OA

In Figure 4.9, the hierarchy of OA is introduced. The obstacle consideration subsystem defines if obstacles should be ignored or not. Furthermore, it defines whether or not obstacles should be combined because of their closeness. The Deliberator, chooses between two avoidance maneuvers: flying around or over the obstacle. Finally, the OA parameters introduced in Chapter 3 are chosen and an "avoiding velocity field" is calculated. The computed parameters are then inputted to the autopilot. In Section 5.1, the subsystems of the OA hierarchy are discussed in more detail. 


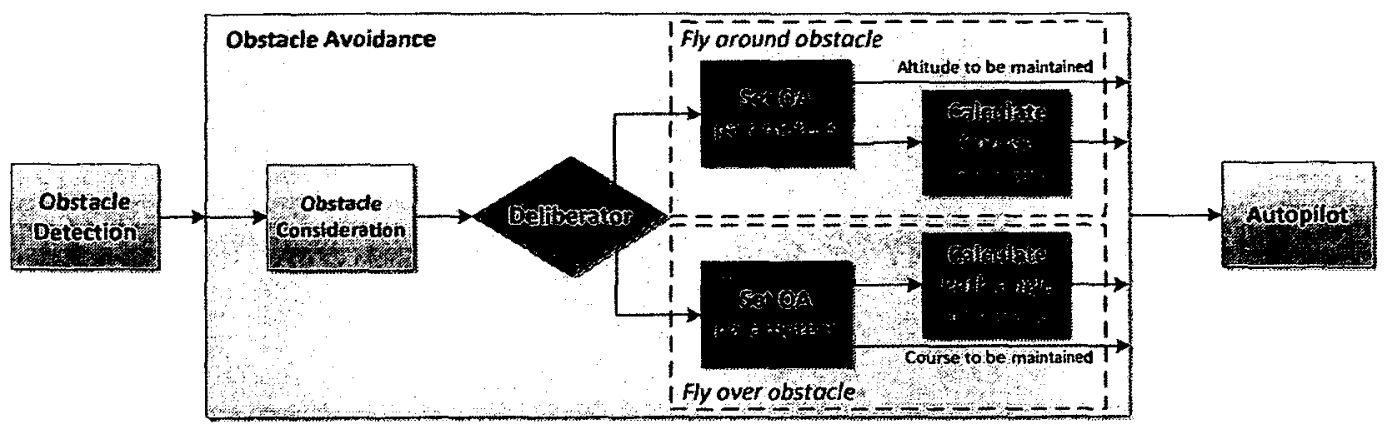

Figure 4.9: Obstacle avoidance hierarchy

\subsubsection{Modified Autopilot Architecture for OA}

When the Selector, as shown in Figure 4.8, has chosen to be in avoidance mode, the autopilot architecture described in Section 4.2 .1 needs to be slightly modified. The high-level controller needs to be redefined such that it can convert avoidance maneuvers to the inputs of the low-level controller. In the modified autopilot, two control laws are used in the high-level controller. The first control law computes a heading command from course error (4.1), while the second one computes a pitch command from path angle error (4.2).

$$
H d g_{c}(t)=k_{p_{c}} \widetilde{C}(t)+k_{i_{c}} \int_{0}^{t} \widetilde{C}(\tau) d \tau+k_{d_{c}} \dot{\widetilde{C}}(t)+C_{c}(t)
$$

where $H d g_{c}$ is the heading command, $C_{c}$ is the course command, $C$ is the actual course and $\widetilde{C}=C_{c}-C$ is the error in course $\left(k_{p_{c}}, k_{i_{c}}, k_{d_{c}}>0\right.$ are constant positive gains).

$$
\operatorname{Pitch}_{c}(t)=k_{p_{p}} \widetilde{P}(t)+k_{i_{p}} \int_{0}^{t} \widetilde{P}(\tau) d \tau+k_{d_{p}} \dot{\widetilde{P}}(t)+P_{c}(t)
$$

where $P$ itch ${ }_{c}$ is the pitch command, $P_{c}$ is the path angle command, $P$ is the actual path angle and $\widetilde{P}=P_{c}-P$ is the error in path angle $\left(k_{p_{p}}, k_{i_{p}}, k_{d_{p}}>0\right.$ are constant 
positive gains).

The modified autopilot is shown in Figure 4.10.

If the Deliberator commands the aircraft to fly around an obstacle, a course command is computed while altitude and airspeed are maintained. Whereas, if the aircraft is to be flown over an obstacle, a path angle command is computed, while course and airspeed are maintained. In Figure 4.10, the command flow of the fly-around and flyover maneuvers are shown in red and green, respectively. Note that the two switches are activated by the Deliberator and depend on which avoiding maneuver is being chosen.

If the high-level controller is not added and the evasive maneuver computed from $\mathrm{OA}$ is commanded as pitch and heading commands directly, the response of the aircraft will worsen. Furthermore, the aircraft would not be able to follow paths in windy situations. To show this fact, the responses of an aircraft are compared when: a desired course is directly used as a heading command, and the desired course is commanded to the modified controller (Figures 4.11 and Figure 4.12, respectively). The same comparison is done for pitch command and path angle (Figures 4.13 and Figure 4.14, respectively).

In Figure 4.11, a heading of 90 degrees is commanded, whereas in Figure 4.12, a 90 degree course is commanded (the flight scenario is illustrated in Figure 4.15). It can be seen that the course error to heading correction control law greatly improves the response time for reaching the commanded course. This is because the control law commands large headings which yields a faster response.

In Figures 4.16 and 4.17 , the scenario of Figure 4.15 is repeated with the addition of a $5 \mathrm{~m} / \mathrm{s}$ wind in the positive $x$ direction. It can be seen that the course of the aircraft has an offset error when the course is directly being used as a heading command (Figure 4.16). This error is due to the fact that the aircraft is flying at a crab angle $(\simeq 10 \mathrm{deg}$ ). The crab angle is shown in Figure 4.18. In contrast, in Figure 4.17, it can 


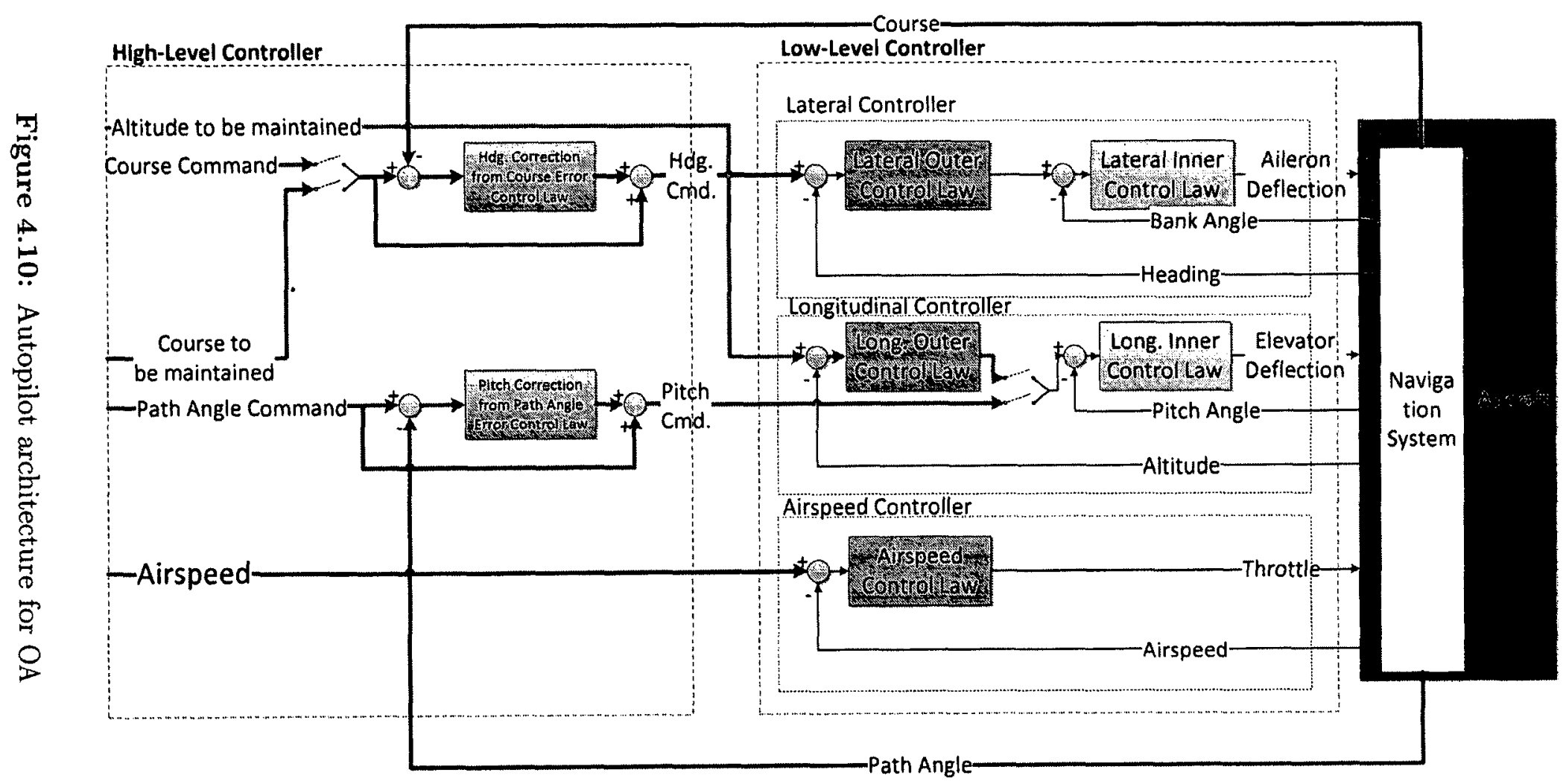



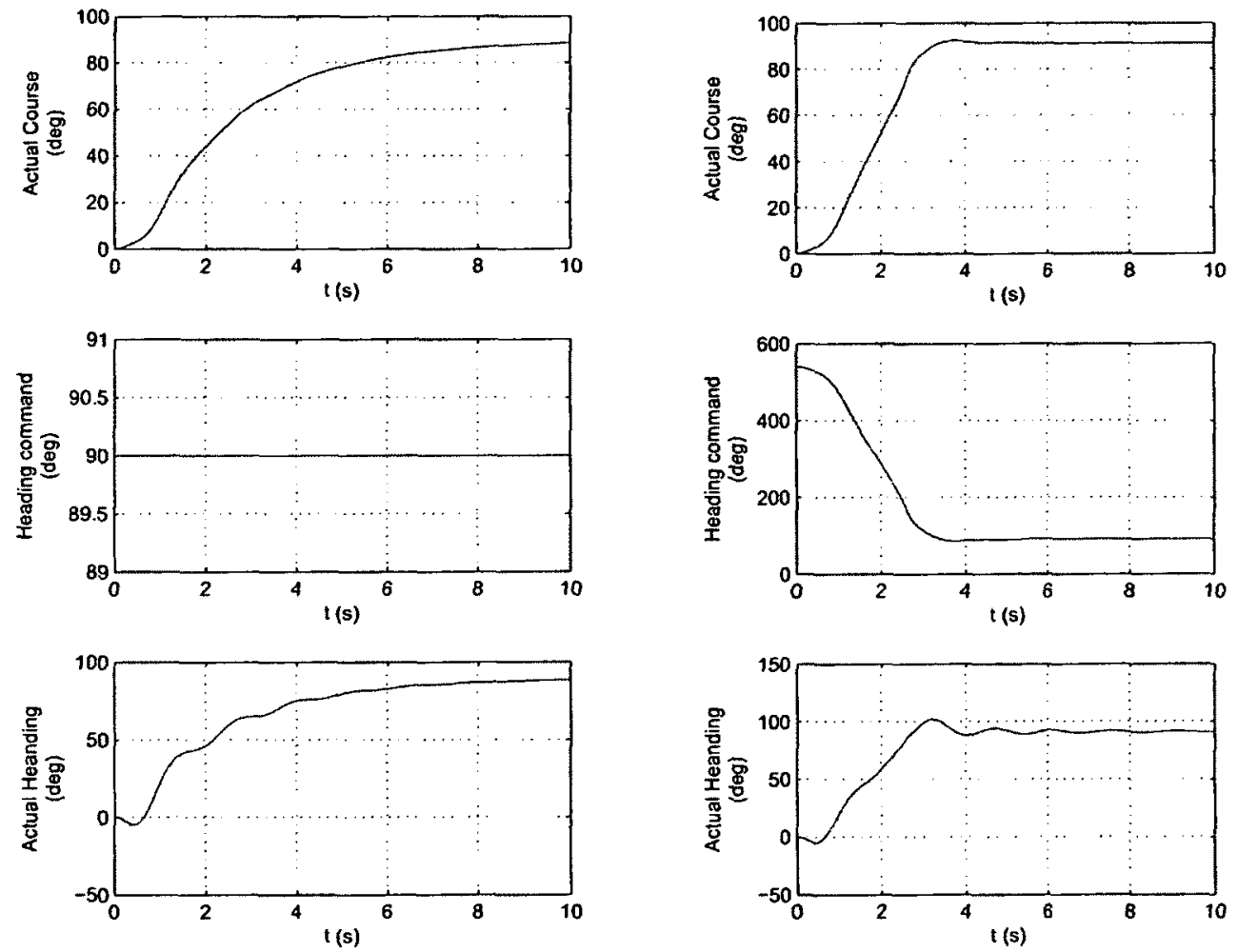

Figure 4.11: +90 degree heading command

Figure 4.12: +90 degree course command 

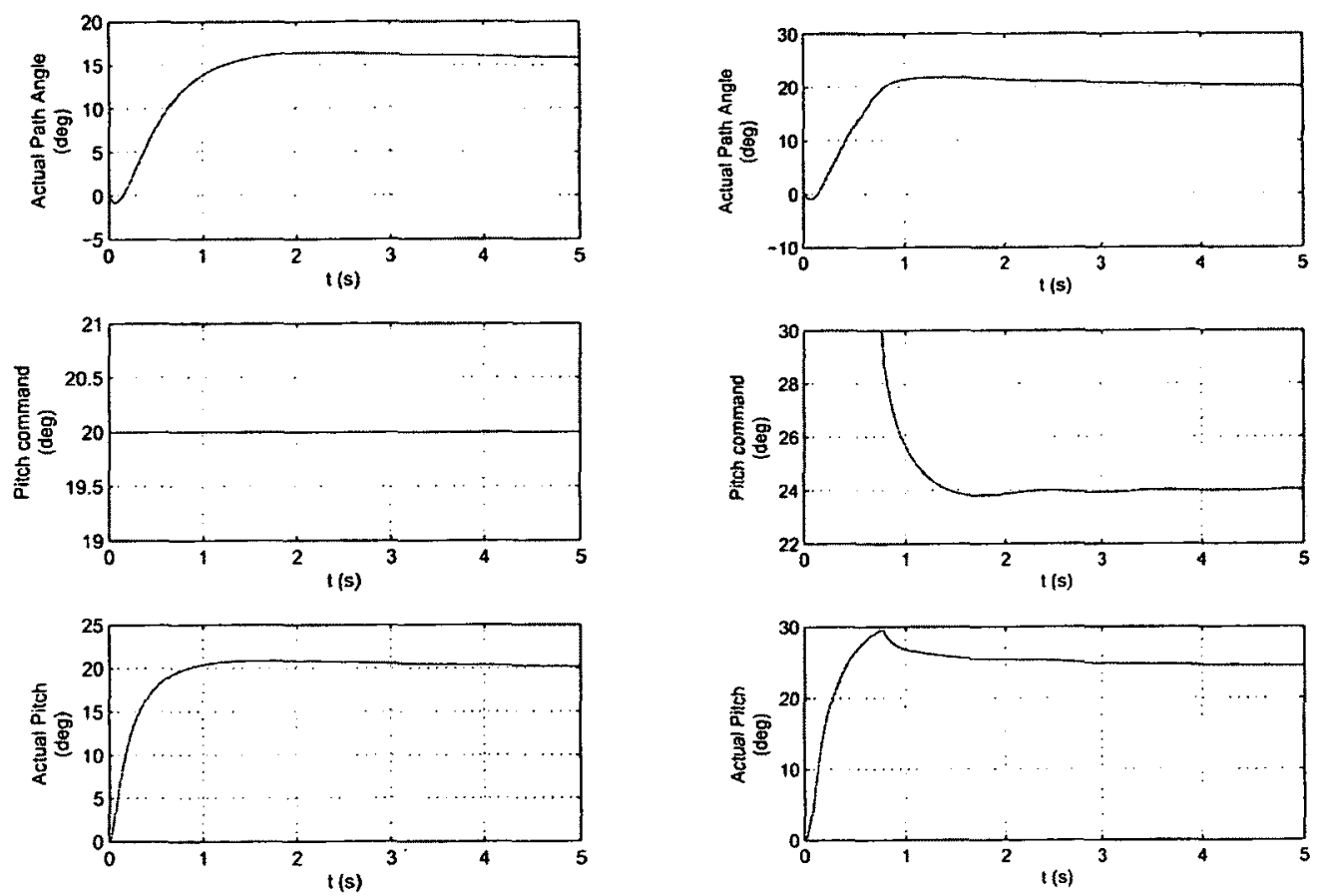

Figure 4.13: 20 degree pitch command

Figure 4.14: 20 degree path angle command
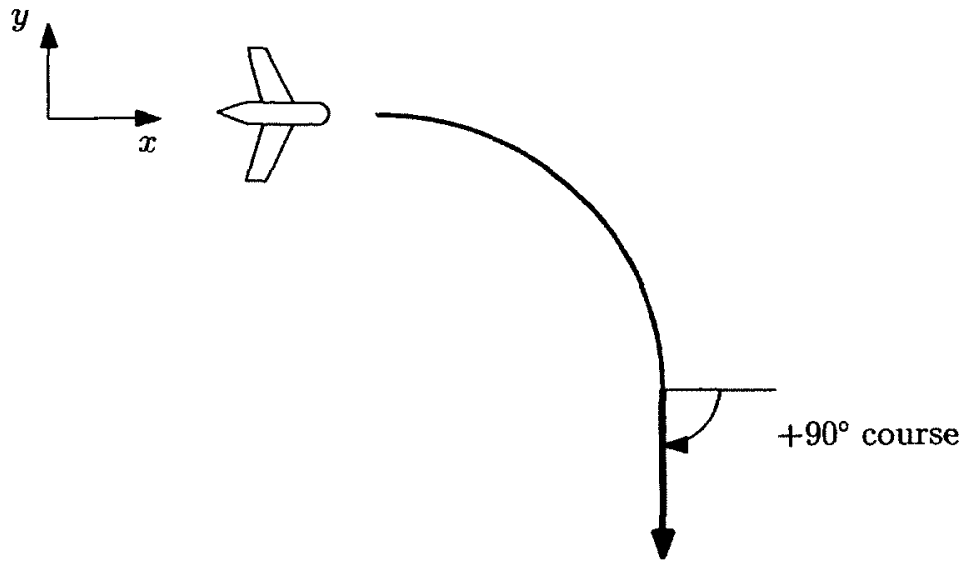

Figure 4.15: +90 degree flight scenario 
be seen that the aircraft successfully tracks the 90 degree course angle.
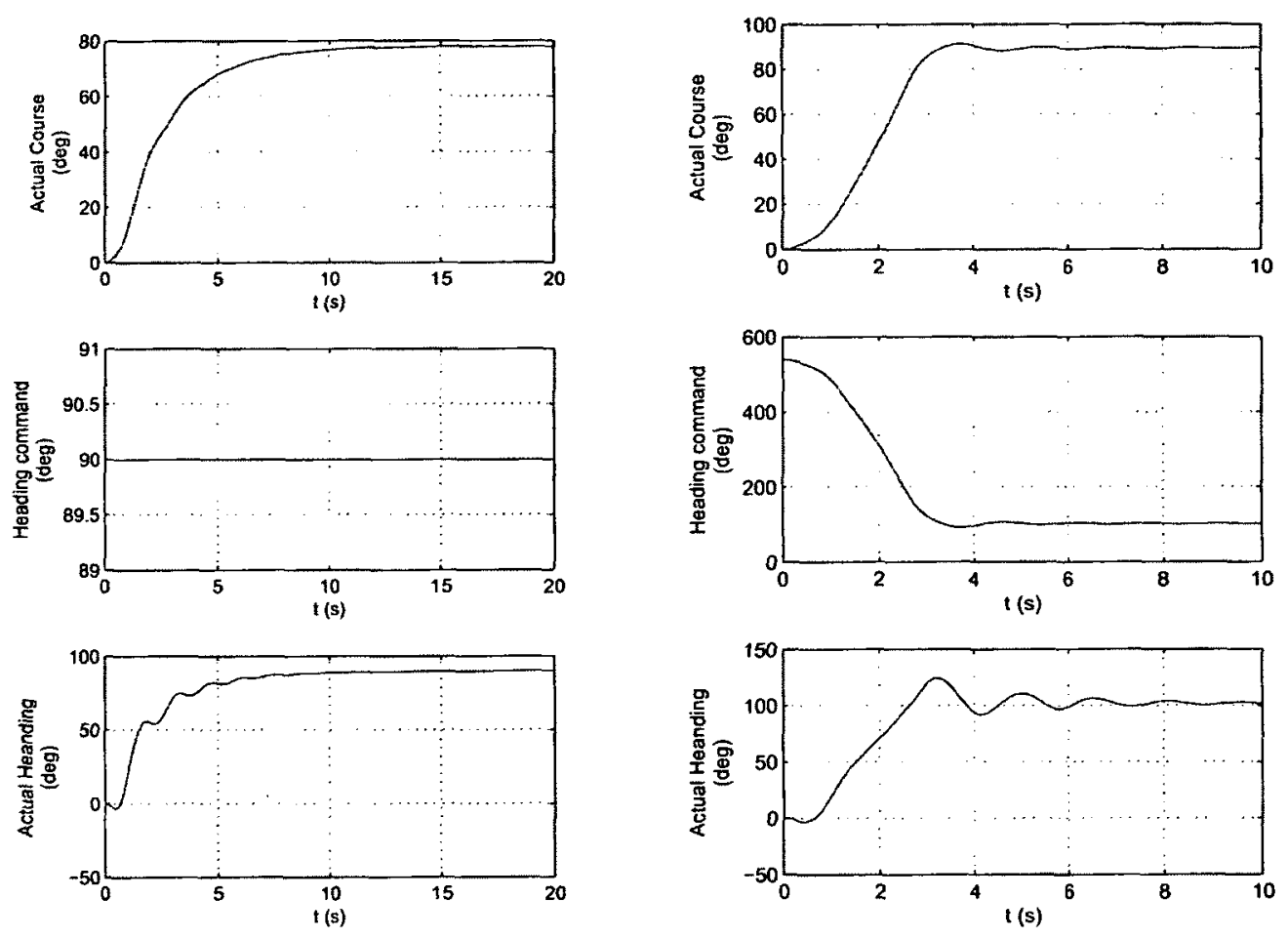

Figure 4.16: +90 degree heading command. Wind $=5 \mathrm{~m} / \mathrm{s}$.

Figure 4.17: +90 degree course command. Wind $=5 \mathrm{~m} / \mathrm{s}$.

A similar trend is seen when the path angle correction controller is added. Comparing Figure 4.13 and 4.14 , and Figure 4.19 and 4.20 , where a $2 \mathrm{~m} / \mathrm{s}$ downdraft is present, shows that without the additional controller the aircraft doesn't follow the intended path.

In Chapter 5 the architecture and simulation framework developed here, will be used to test the OA method introduced in Chapter 3. 


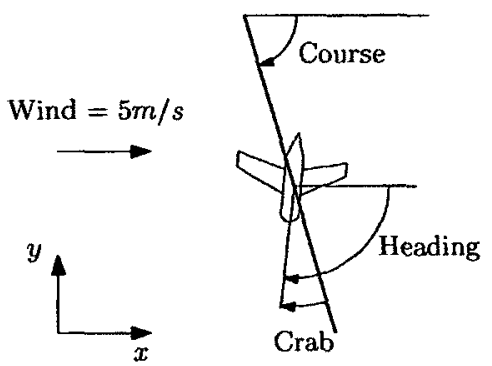

Figure 4.18: Crab angle while flying in wind
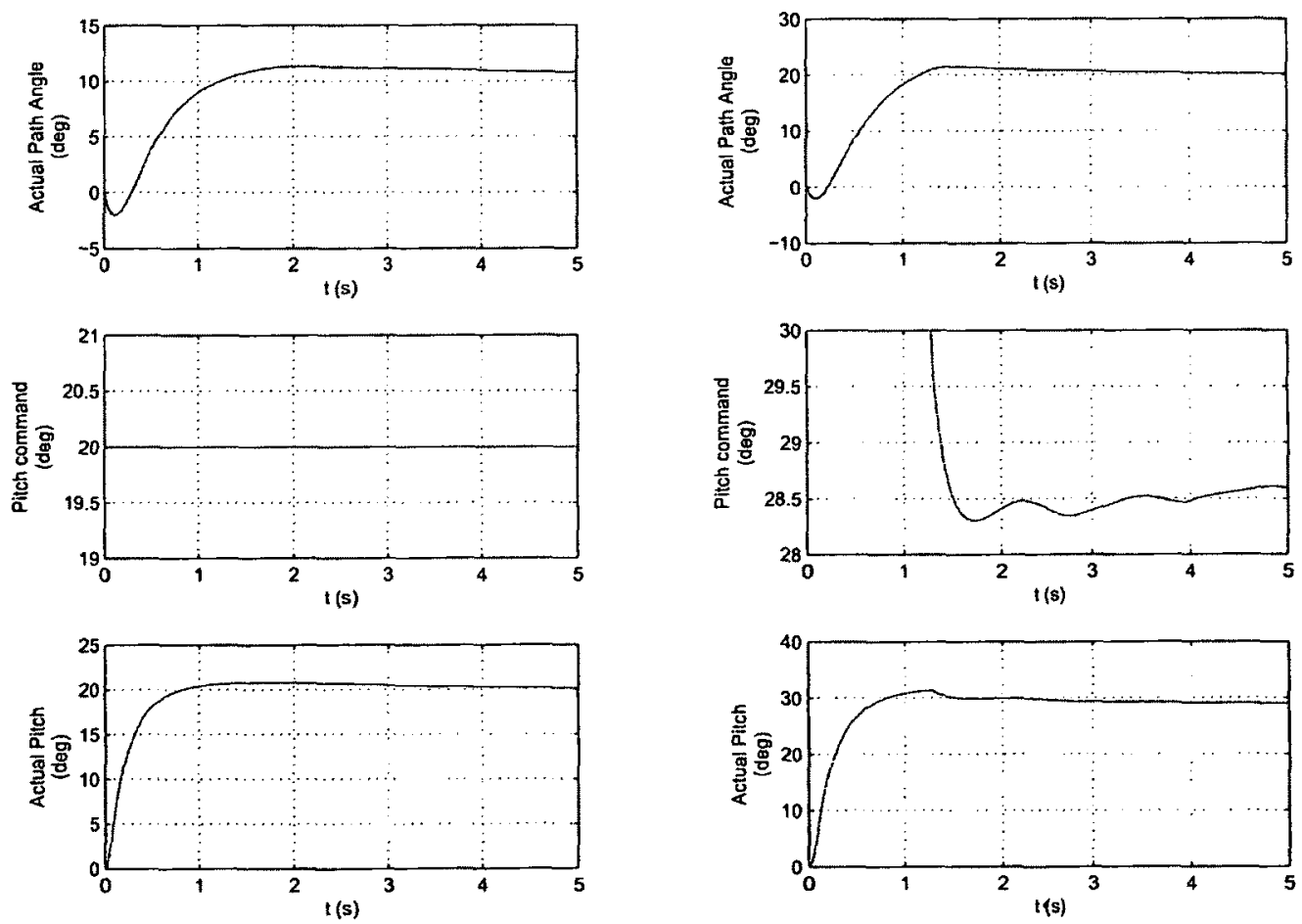

Figure 4.19: 20 degree pitch command with $2 \mathrm{~m} / \mathrm{s}$ downdraft

Figure 4.20: 20 degree path angle command with $2 \mathrm{~m} / \mathrm{s}$ downdraft 


\section{Chapter 5}

\section{Obstacle Avoidance Implementation \& Results}

\subsection{Obstacle Avoidance Implementation}

In order to test avoidance maneuvers the subsystems of the OA hierarchy introduced in Section 4.3 are first defined. Furthermore, the Selector's choice making process will be discussed.

\subsubsection{Obstacle Consideration}

The goal of obstacle consideration is to define how obstacles should be dealt with. For instance, how close should two obstacles be in order to be considered as one obstacle, and how far away should an obstacle be in order for it to be ignored. After the Considered Obstacle $(\mathrm{CO})$ is defined, wedges are added to it to incorporate aircraft constraints (Section 3.5.2.1). The resulting obstacle is called the Modelled Obstacle (MO).

Obstacle consideration is performed in two planes: the top and side plane. The former is used for a fly-around maneuver, while the latter is employed for a fly-over maneuver. The top plane is defined as a plane intersecting with the nominal flight 
path and parallel to the ground plane. On the other hand, the side plane is a plane intersecting with the nominal flight path, but perpendicular to the ground plane. The avoiding maneuver is defined such that it avoids the intersections of the three dimensional obstacles with these planes. In Figure 5.1, an aircraft encountering an obstacle scenario is shown. The top and side plane with the obstacle section views are shown in Figure 5.2 and Figure 5.3, respectively ${ }^{1}$.

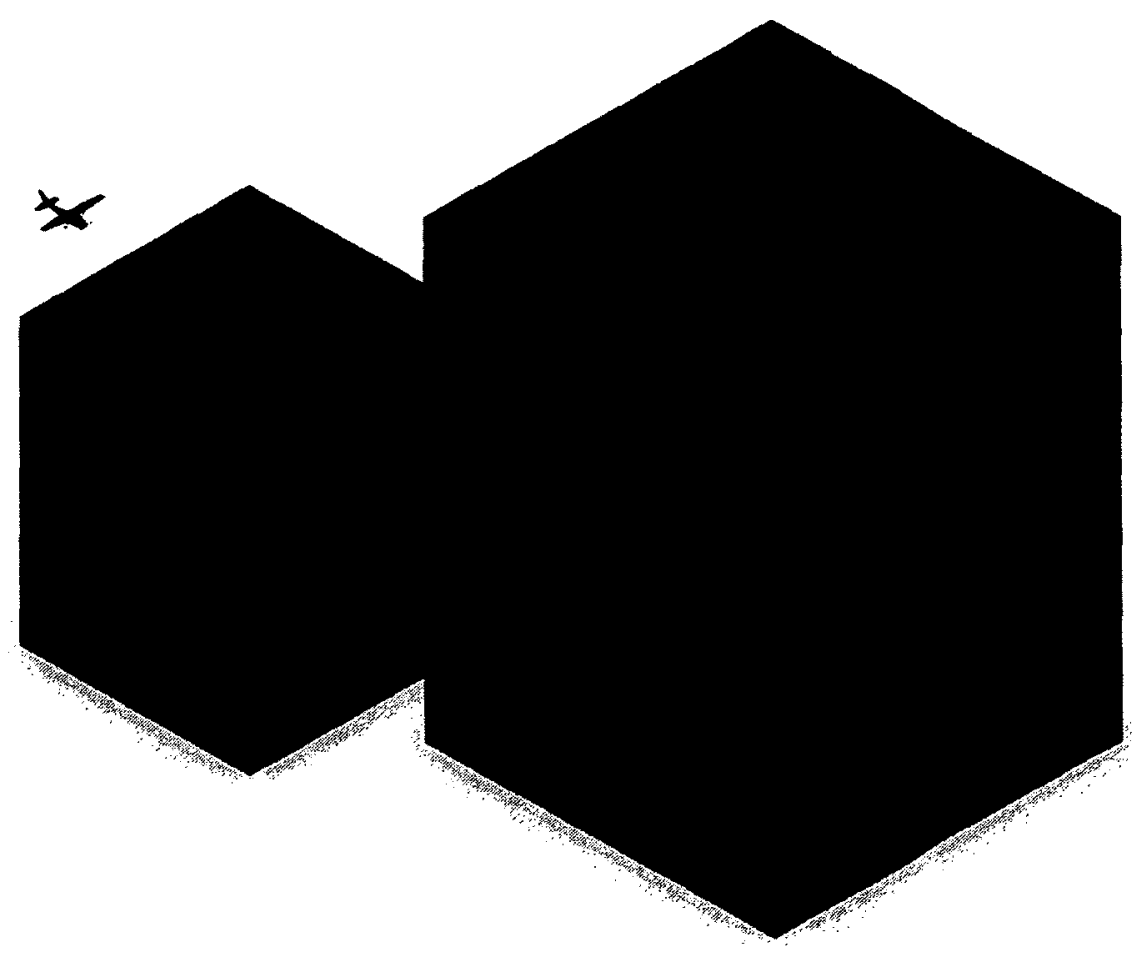

Figure 5.1: Three-dimensional view of an aircraft encountering an obstacle scenario

\subsubsection{Top Plane Obstacle Consideration}

To prevent the aircraft from flying very close to the boundaries of obstacles, the detected obstacles are artificially magnified. The factor of magnification proposed is

\footnotetext{
${ }^{1}$ From here on the section views of the three dimensional obstacles will be called obstacles.
} 


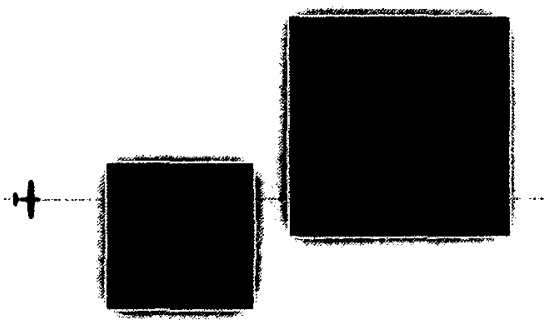

Figure 5.2: Top plane obstacle scenario

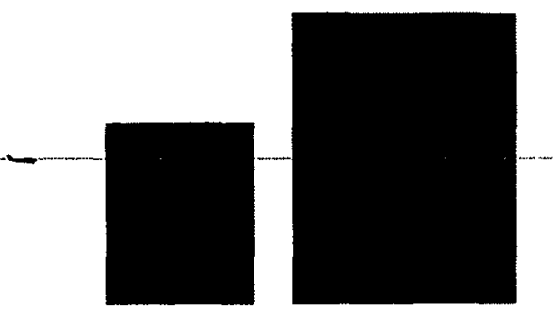

Figure 5.3: Side plane obstacle scenario

a multiplication, $n_{S F}>0$, of the aircraft's wingspan, $b$. Therefore, the obstacle's new width is

$$
a=a+2 n_{S F} b
$$

This results in the aircraft to always fly at least $n_{S F}$ wingspans away from the obstacle. The choice of $n_{S F}$ affects how conservative the avoidance strategy is. A high value of $n_{S F}$ suggests a flight trajectory that is further away from the obstacle, whereas a lower value results in a flight that follows the obstacle more closely. The value of $n_{s F}$ is a safety factor and can be chosen accordingly.

Explicit and Implicit Obstacles While performing an evasive maneuver, obstacles that lie on the aircraft's nominal path, i.e. the "explicit" obstacles, must be considered for avoidance. Moreover, the other "implicit obstacles" (that do not lie on the nominal path) might also need to be considered. With regards to the implicit obstacles, one of three actions is taken: (1) the implicit obstacle is combined with the explicit obstacle and a bigger obstacle is created, (2) the implicit obstacle is ignored, or (3) the explicit and implicit obstacles are considered simultaneously. In order to decide which action to take, an Avoidance Range (AR) is defined around each obstacle. Based on whether or not obstacles and their ARs intersect, the action to be 
taken is chosen.

The AR is defined as an area that encompasses all evasive pathlines that correspond to the avoidance of an explicit obstacle, and is shown in Figure 5.4 for an obstacle in the top plane. The avoidance range is specified by the parameters $y_{m a x}$ and $d$, as shown, as well as the obstacle size given by its width, $a$ and wedge length, $h$. As shown in Figure 5.4, $y_{\max }$ is the maximum lateral distance from the horizontal sides of an obstacle, which corresponds to the pathlines starting on the extreme limits of the explicit obstacle. The value of $y_{\max }$ depends on the wedge length of the

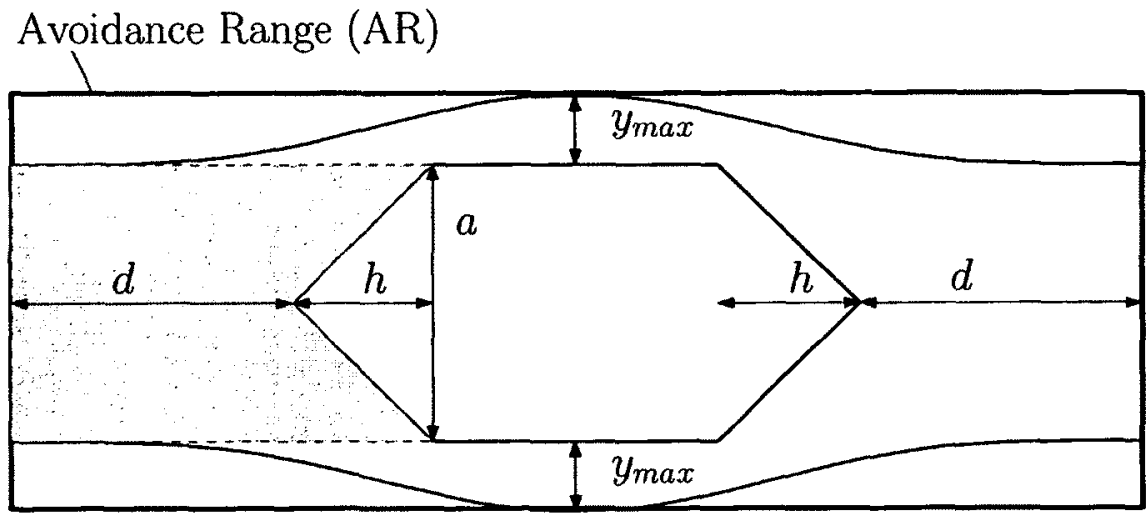

Figure 5.4: Obstacle Avoidance Range (Top view)

obstacle being modelled.

This correlation is plotted in Figure 5.5. In order for the AR to be conservative, the maximum value of $y_{\max }$, which corresponds to a zero wedge length is chosen. Therefore

$$
\frac{y_{\max }}{a}=0.421
$$

Finally, $d$ will be defined in Section 5.1.3 and the method to compute $h$ is explained in Section 5.1.4.1.

If an implicit obstacle intersects with the AR of an explicit obstacle, it means that some of the avoiding pathlines of the explicit obstacle, may intersect the implicit obstacle. Therefore, the obstacles are combined to create a $\mathrm{CO}$. Such a scenario is 


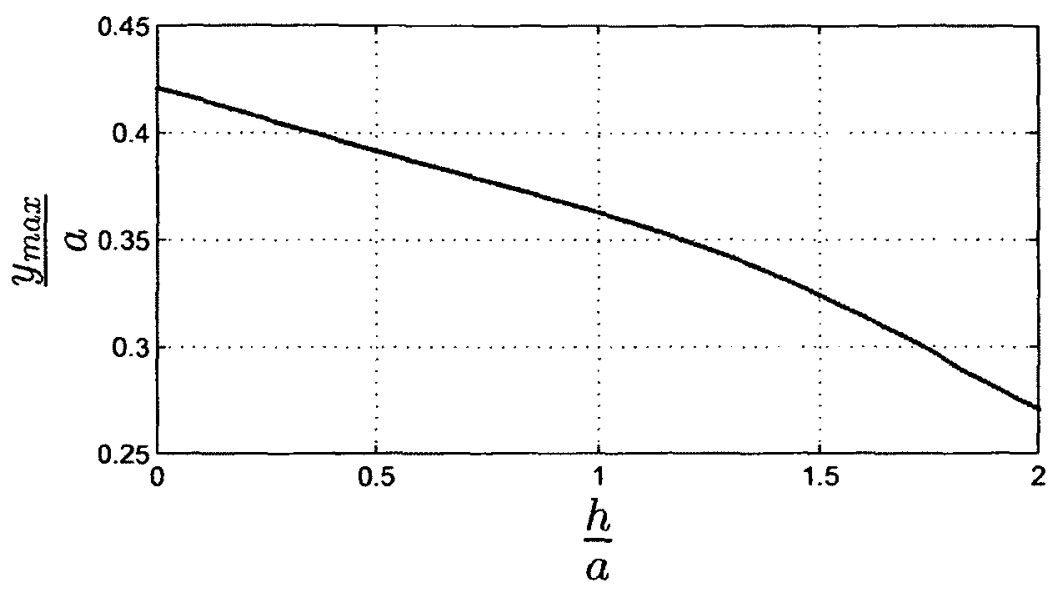

Figure 5.5: Correlation between $y_{\max }$ and wedge length, $h$

shown in Figure 5.6. Note that the $\mathrm{CO}$ is a rectangle that encompasses both obstacles. In Figure 5.6, $\delta_{y}$, which will be needed in Section 5.1.2, is defined as the minimum distance along the $y$ axis from the sides of the $\mathrm{CO}$.

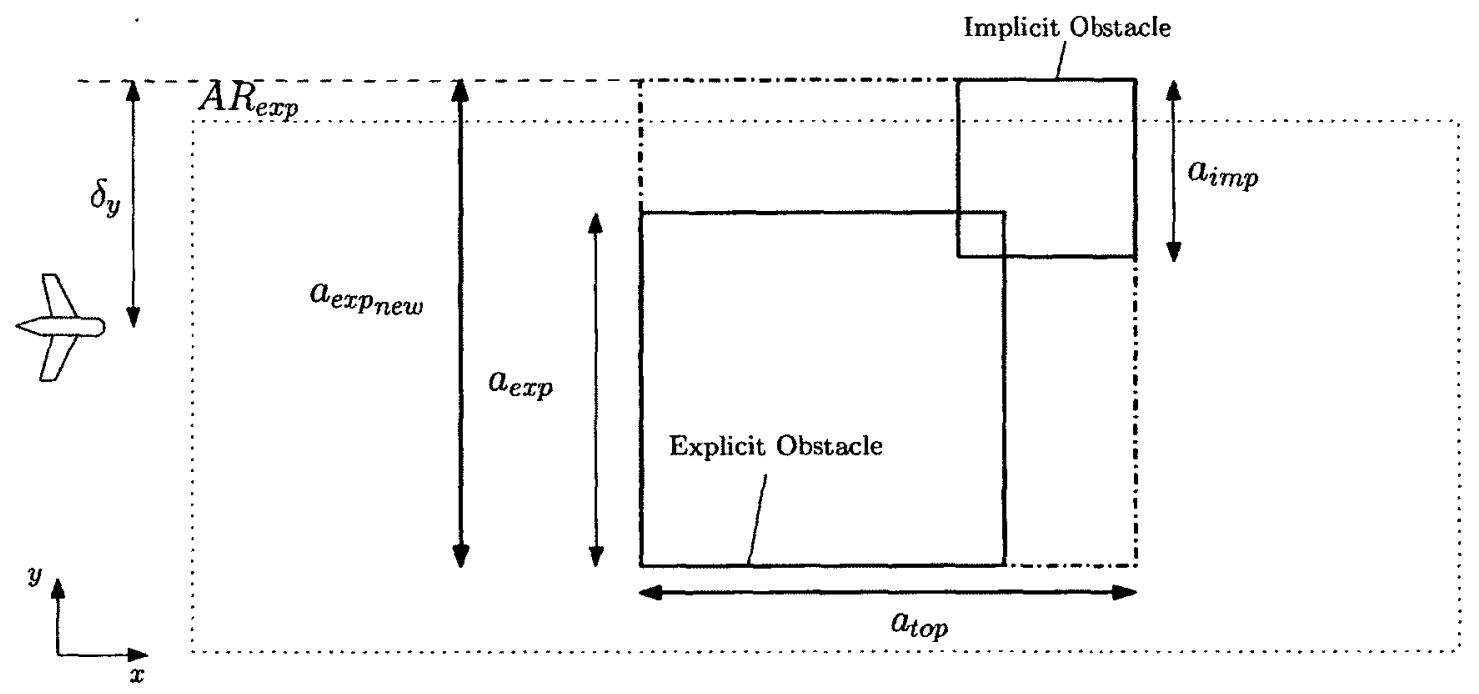

- Figure 5.6: Combining explicit and implicit obstacles (top plane) .

The second case is when an implicit obstacle itself does not intersect with the AR of an explicit obstacle, but its AR does. This means that even though the implicit obstacle does not intersect with any avoiding pathline of the explicit obstacle, it is still 
in close proximity. Therefore, the implicit obstacle is also considered as a separate obstacle. By doing this, if the aircraft is pushed out of the explicit obstacle's AR (for instance by a wind gust), the avoidance maneuver is considering the implicit obstacle and therefore, the aircraft will, avoid the implicit obstacle. This scenario is illustrated in Figure 5.7.

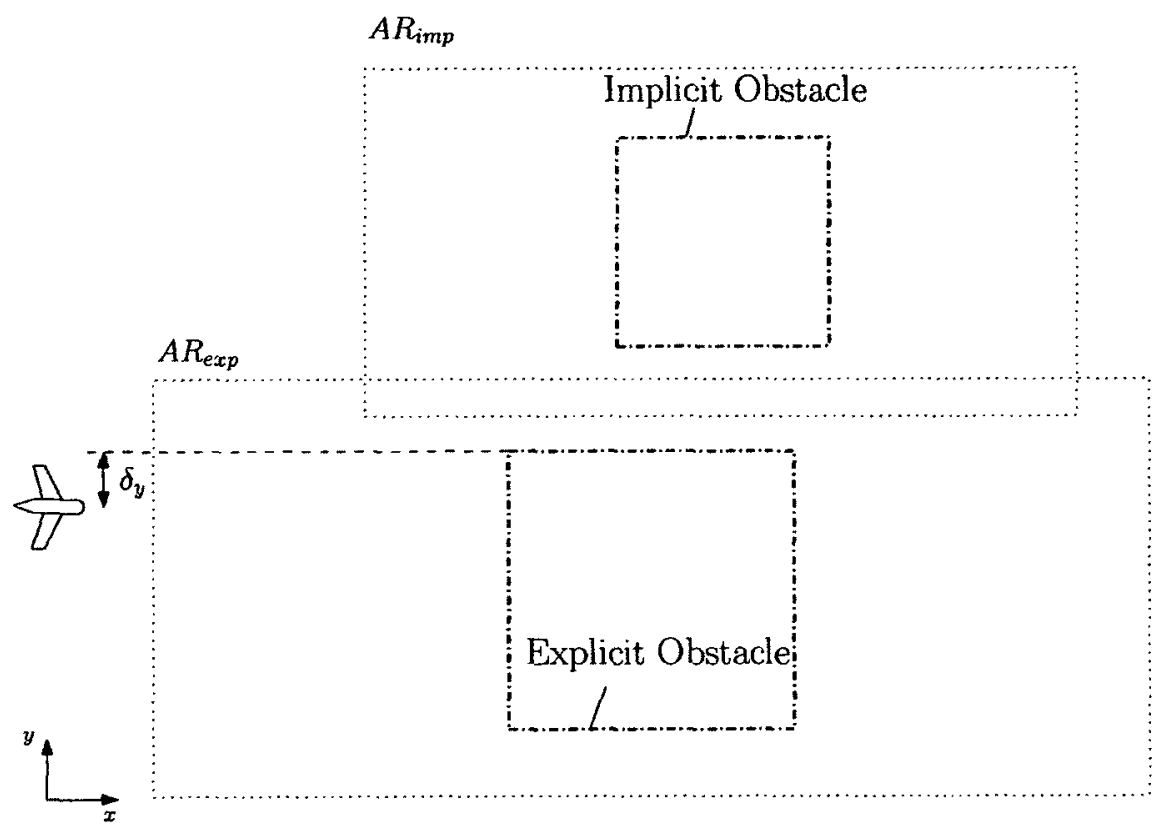

Figure 5.7: Simultaneously considering an explicit and implicit obstacle

Finally, when the AR of an implicit obstacle does not intersect that of an explicit obstacle, the implicit obstacle is ignored.

Multiple Explicit Obstacles In the event of multiple explicit obstacles only one of two actions is taken: combining the obstacles or temporarily ignoring the obstacles further away. Considering obstacles simultaneously can lead to commanding trajectories with smaller turning radii, than the aircraft is capable of performing. Such a scenario is shown in Figure 5.8. Note that the turns of the pathlines between the two obstacles are much tighter than the turns before the first obstacle and after the last obstacle. 


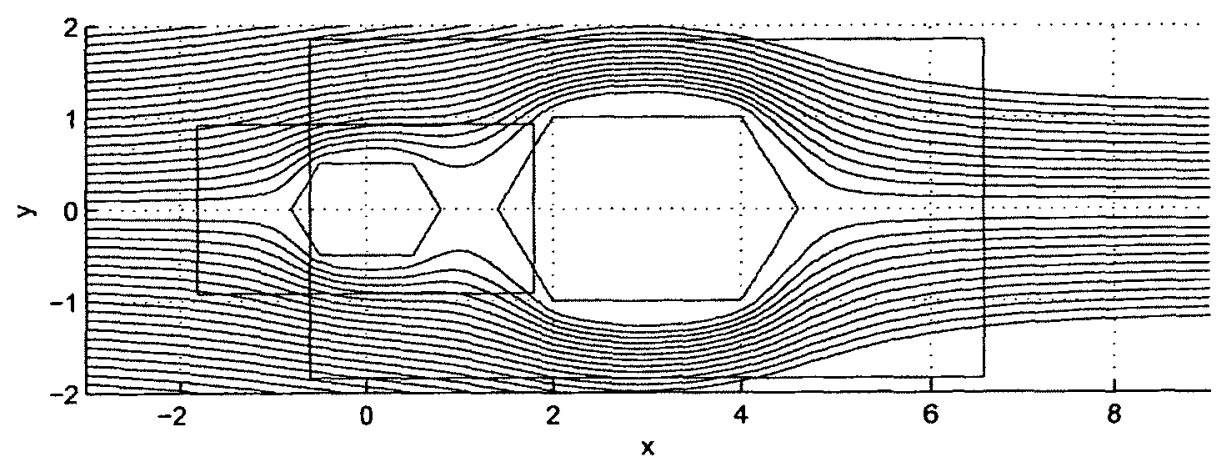

Figure 5.8: Simultaneously considering two explicit obstacles

Therefore, if the ARs of explicit obstacles intersect, the obstacles are combined. In contrast, if the ARs do not intersect at all, the secondary obstacle is ignored until the first obstacle is successfully avoided. Then, the second obstacle is treated as a separate obstacle.

For all cases, after the $\mathrm{CO}$ is defined, if the aircraft is in the $\delta$-region of the $\mathrm{CO}$, the obstacle is resized according to Section 3.5.2.1.

\subsubsection{Side Plane Obstacle Consideration}

The side plane obstacle consideration is similar to that of the top plane with some important differences. Firstly, the obstacle magnification is different. Secondly, the aircraft is always required to fly over obstacles ${ }^{2}$. This is because a "fly-under" avoidance maneuver might intersect with the ground which will lead to crashing. Furthermore, even if the avoiding pathlines do not intersect with the ground, the aircraft is lowering its altitude and therefore highly exposing itself to additional potential obstacles.

Similar to Section 5.1.1.1, in order to prevent the airplane from flying close to the boundaries of obstacles, the obstacle height, $H$, is artificially increased such that

$$
H=H+n_{s F} b
$$

\footnotetext{
${ }^{2}$ However flying under implicit obstacles is tolerated
} 
for obstacles that are directly connected to the ground such as trees or buildings and

$$
H=H+2 n_{s F} b
$$

for other obstacles such as power lines (see Figure 5.9). Note that $b$ is the aircraft's

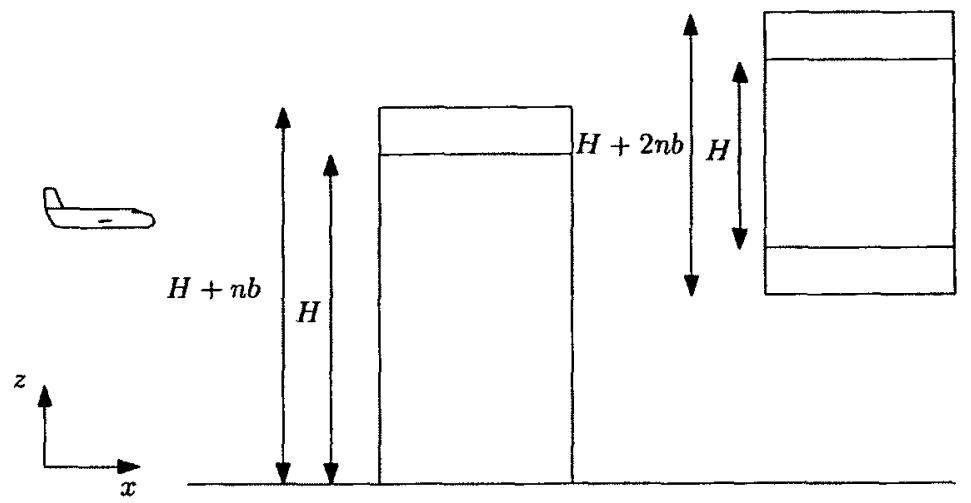

Figure 5.9: Artificially magnifying obstacles in the side plane

wingspan and $n_{S F}$ is a safety factor, which is chosen to be equal to that of the top plane obstacle consideration.

The same procedure as in the top plane is used for multiple obstacles. Note that instead of $a, \delta_{y}$ and $a_{\text {top }} ; H, \delta_{z}$ and $a_{\text {side }}$ are used (see Figure 5.10).

In order to keep the aircraft from flying under obstacles, one has to make sure that the aircraft is always above the centerline (and outside of the $\delta$-region). Therefore, after the $\mathrm{CO}$ is obtained, if

$$
\delta_{z}>0.4 H
$$

the obstacle needs to be resized to guarantee the aircraft will not attempt to fly under the obstacle (see Figure 5.11).

From (3.68), $H$ is set to

$$
H=1.25\left(2 \delta_{z}\right)=2.5 \delta_{z}
$$




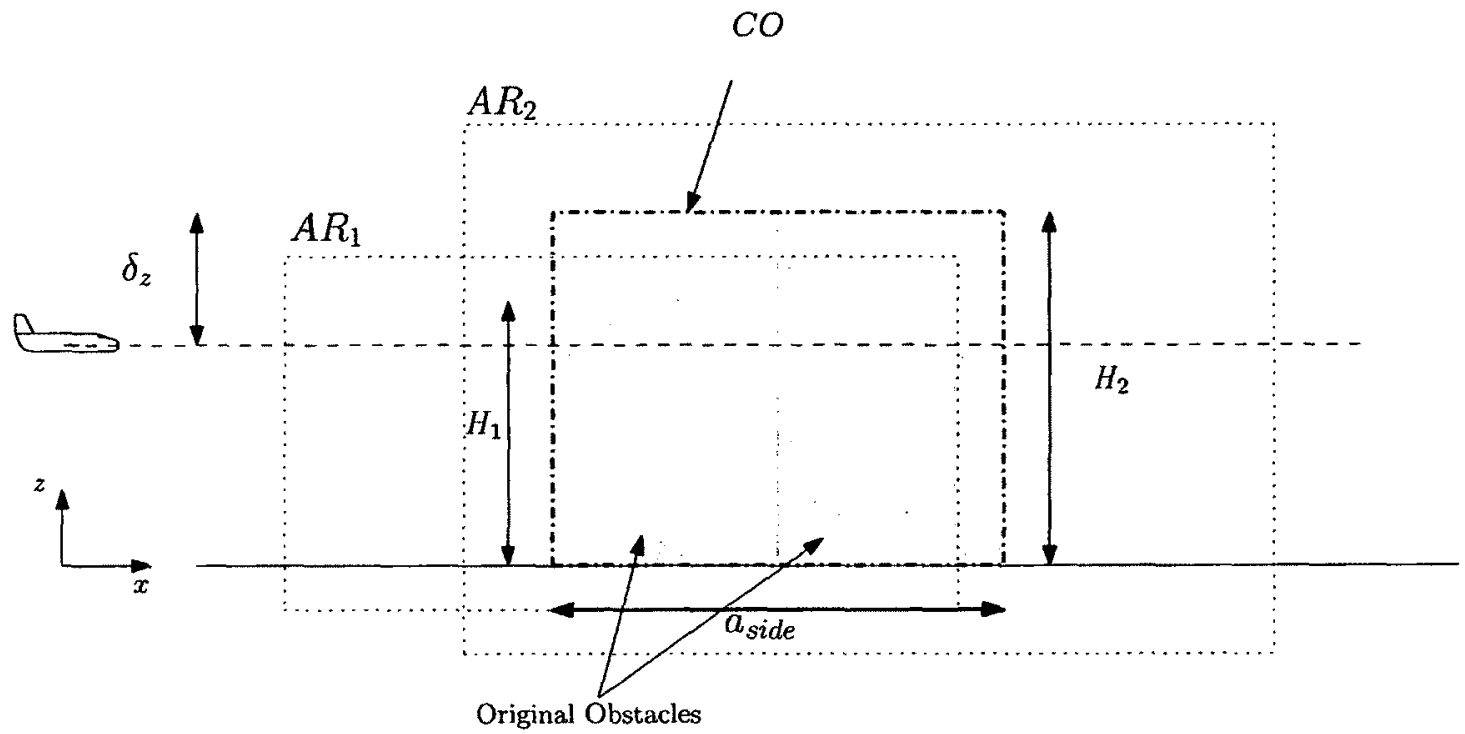

Figure 5.10: Combining two explicit obstacles (side plane)

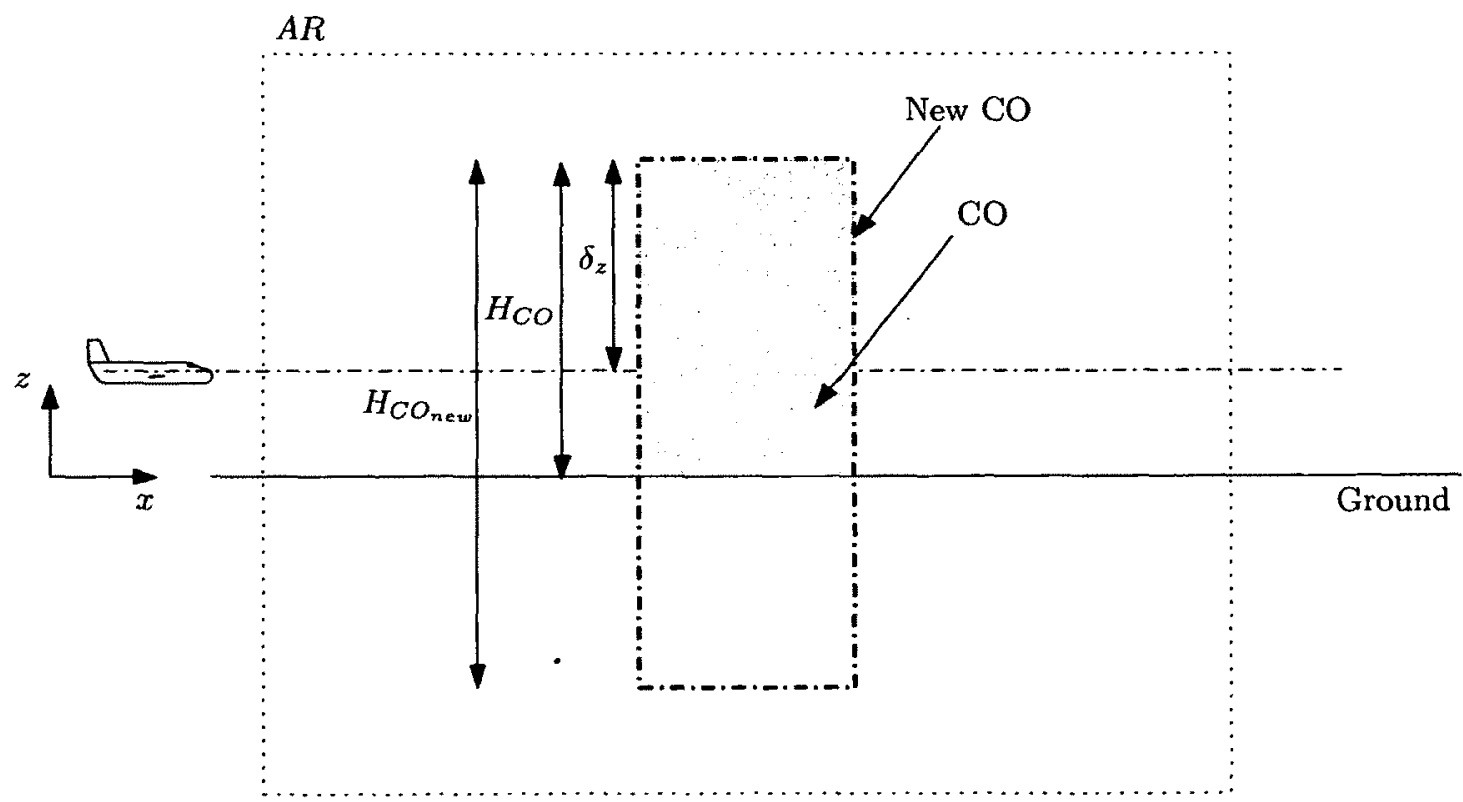

Figure 5.11: Resizing obstacles in the side plane to guarantee flying over the obstacle 
This results in the aircraft being above the centerline and outside of the $\delta$ region.

\subsubsection{Deliberator}

When an avoiding maneuver is required, the Deliberator has to choose between a fly-around and fly-over maneuver. This decision is made by choosing the maneuver that has less deviation from the aircraft's original flight path.

The projection of the avoiding maneuver on the obstacle is used to approximate the amount of deviation. The path with the shortest projection is selected as the avoidance maneuver. From Figure 5.12, if

$$
a_{\text {side }}+2 \delta_{y}<a_{\text {top }}+2 \delta_{z}
$$

the projection of the fly-around maneuver is shorter than that of the fly-over. Therefore, a fly-around maneuver is chosen, otherwise, a fly-over is selected.

The condition checked in the Deliberator (5.7), may be tailored based on the nature of the mission being performed. For instance, a change of altitude to a certain extent might be tolerated for a UAV because the data being measured (as part of the UAV's mission) remains acceptable. Therefore, this would be incorporated in the condition of the Deliberator. Or one might want to consider the wedge lengths required in the condition of the Deliberator. However, in this thesis, the Deliberator condition was chosen such as (5.7) for simplicity.

It should be noted that $a_{\text {top }}$ and $a_{\text {side; }}$, defined in Figure 5.6 and 5.10, are not always equal. For instance, an implicit obstacle might have to be combined with an explicit obstacle in the top plane, but not in the side plane. Such a scenario will be shown in Section 5.2.3. 


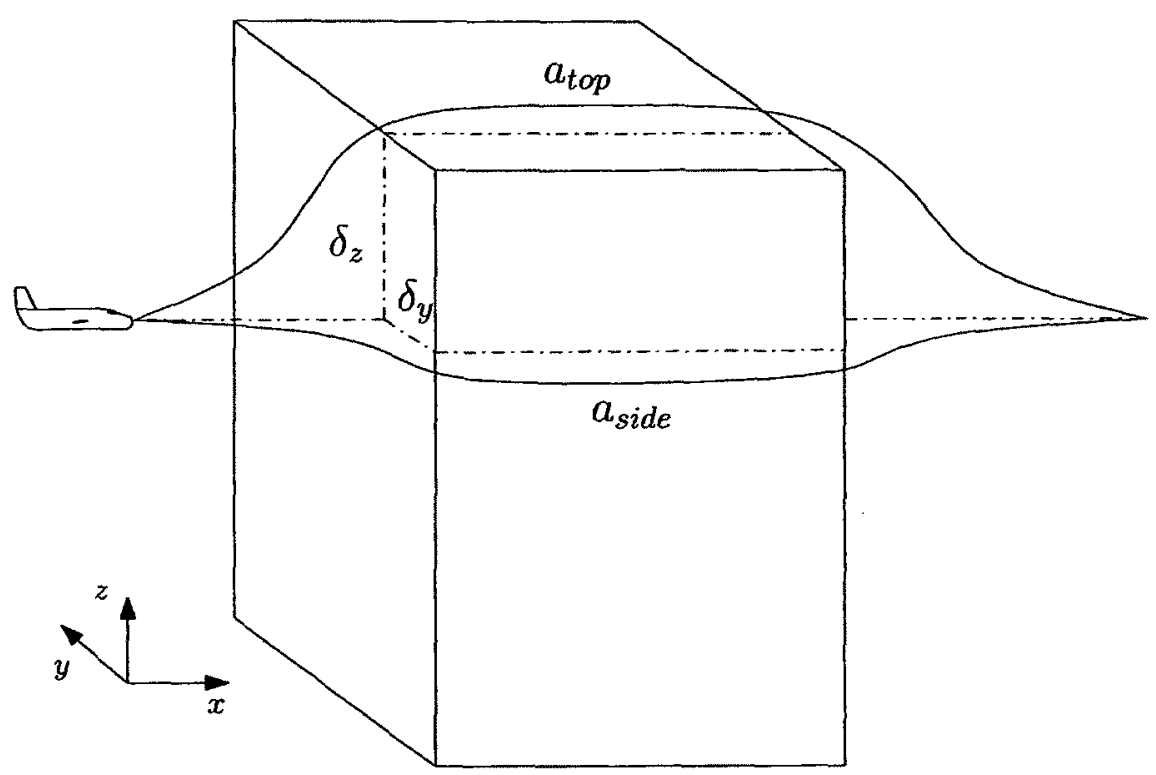

Figure 5.12: Projection of avoiding maneuver on obstacle

\subsubsection{Selector}

The Selector chooses when to engage OA to perform and start an evasive maneuver. When flying, the nominal flight path of the aircraft is constantly checked for intersection with obstacles. If such an intersection is detected, the Selector needs to engage OA. However, in order to keep the aircraft in non-avoidance mode as long as possible, a limit has to be defined as to when OA should engage. This limit will be called an Activation Area (AcA) and is shown in Figure 5.13 in the top plane.

It is desirable for the avoiding velocity field to be parallel to the nominal flight path on the line of entry to the AcA. Note that this means zero course angle for a fly-around maneuver and zero path angle for a fly-over maneuver. If this property is not satisfied, the aircraft would be commanded to reach an initial course/path angle instantly after entering the AcA. Due to the fact that the aircraft cannot fulfill such a command instantly a gradual increase in the command from zero is desired.

However, even for very large distances from the obstacle, the velocity field will never have zero course/path angle. In Figure 5.14, the maximum course/path angle 


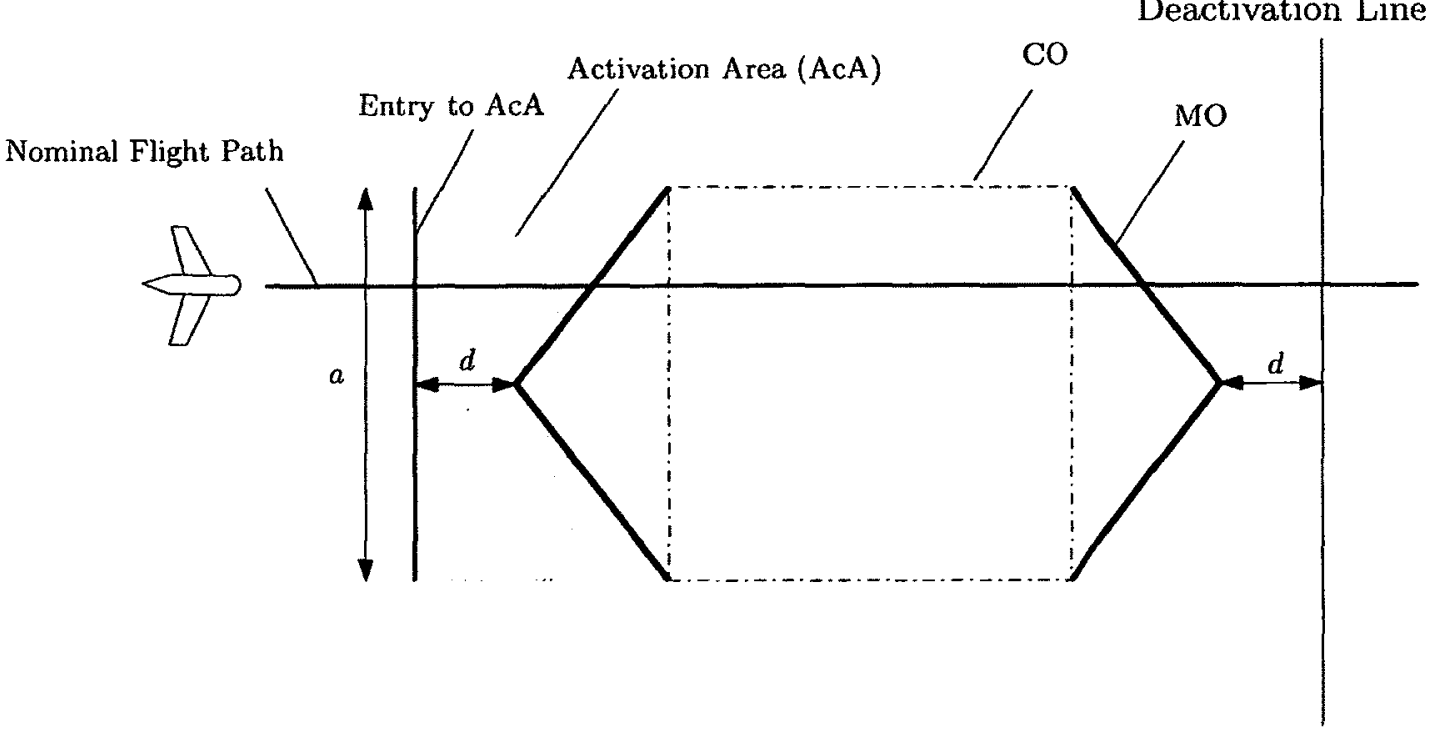

Figure 5.13: Activation Area (AcA) in the top plane

at the the entry line to the AcA is shown for different values of $d / a$. Note that the different curves correspond to different values of $h / a$. Furthermore, the higher $d / a$ is, the lower the entry course/path angle is.

The value of $d$ is set to $d=a$, where the maximum path/course angle, which corresponds to a zero wedge length, is $6.05^{\circ}$. The Selector maintains OA active until it crosses the Deactivation Line shown in Figure 5.13. The Deactivation line is also chosen to be at a distance of $d=a$ from the obstacle's wedge tip. As a result, the aircraft will leave avoidance mode with a course/path angle that is close to zero.

Finally, it should be noted that the Deliberator decides which AcA, i.e. the top plane or the side plane, is used by the Selector.

Similar to the concept developed here, the limits of the AR in Section 5.1.1.1 are - defined such that $d=a$. 


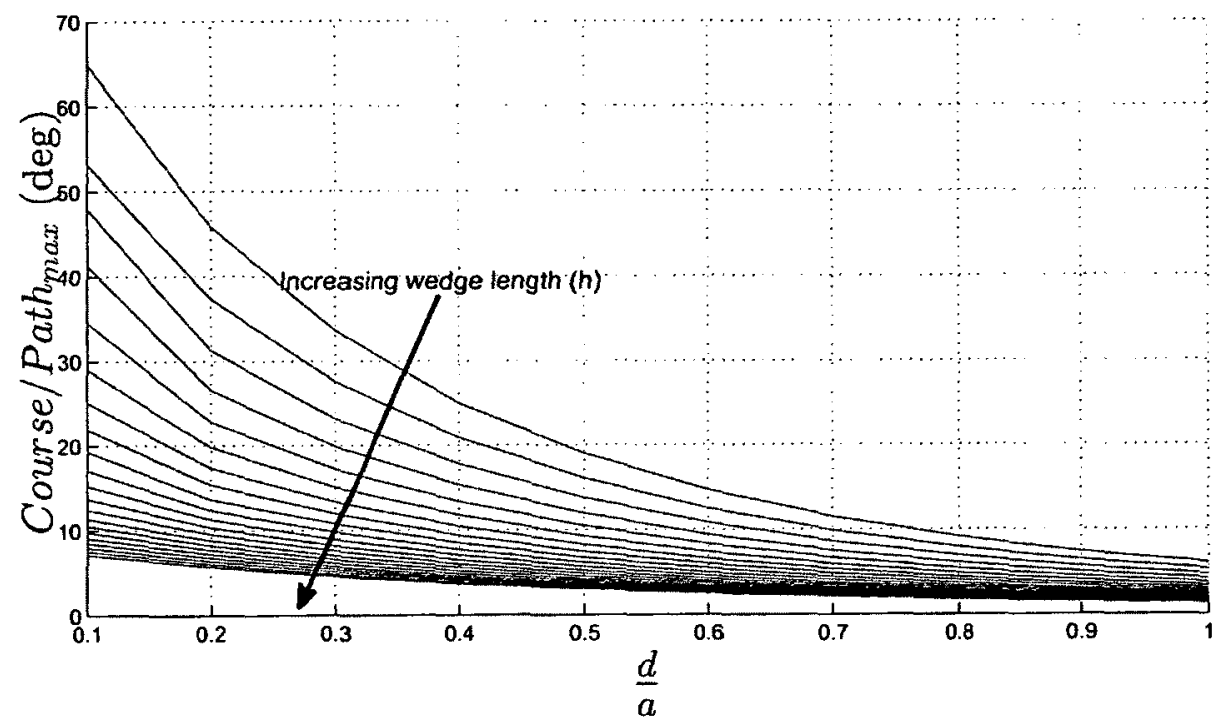

Figure 5.14: Maximum course/path angle $(0<h / a<2$ with 0.1 increments $)$

\subsubsection{Setting OA Parameters}

In order to compute the velocity field around an obstacle, three parameters need to be set: $V_{\infty}$ in (3.41), $\Lambda_{g}$ and $\left(x_{g}, y_{g}\right)$ in (3.69) for a fly-around maneuver and $\left(x_{g}, z_{g}\right)$ for a fly-over maneuver.

In Section B.4 it is shown that in the absence of a sink, the value of $V_{\infty}$ does not affect the pathlines around an obstacle. Therefore, without loss in generality, the free-stream velocity is taken to be

$$
V_{\infty}=1 \mathrm{~m} / \mathrm{s}
$$

The sink is set to lie on the nominal flight path. Therefore for a fly around maneuver,

$$
y_{g}=y \text { (nominal flight path) }
$$


and for a fly-over maneuver,

$$
z_{g}=z \text { (nominal flight path) }
$$

Recall that the reason a sink is added to the potential flow is to attract all pathlines back to the nominal flight path. To calculate $\Lambda_{g}$ from (3.79), $l$ is set such that all pathlines that are within the ARs of $\mathrm{CO}(\mathrm{s})$ lie within

$$
\left(y_{g}-l / 2, y_{g}+l / 2\right)
$$

for a fly-around maneuver and within

$$
\left(z_{g}-l / 2, z_{g}+l / 2\right)
$$

for a fly-over maneuver.

From Figure 5.15, for a fly-around maneuver

$$
l=2 \times \max (\alpha, \beta)
$$

and

$$
x_{g}=\max \left(x_{\max }\left(A R_{e x p}\right), x_{\max }\left(A R_{i m p}\right)\right)+\epsilon
$$

where $\epsilon$ is a very small value. $\epsilon$ is added to set the sink outside of the zone where OA is being performed. This is done to avoid the singularity of the sink itself. Furthermore, the "returning effect", shown in Figure 5.16, will be outside of the effective OA zone.

Similarly, for a fly-over maneuver, $l$ and $x_{g}$ are computed from (5.11) and (5.12), respectively. 


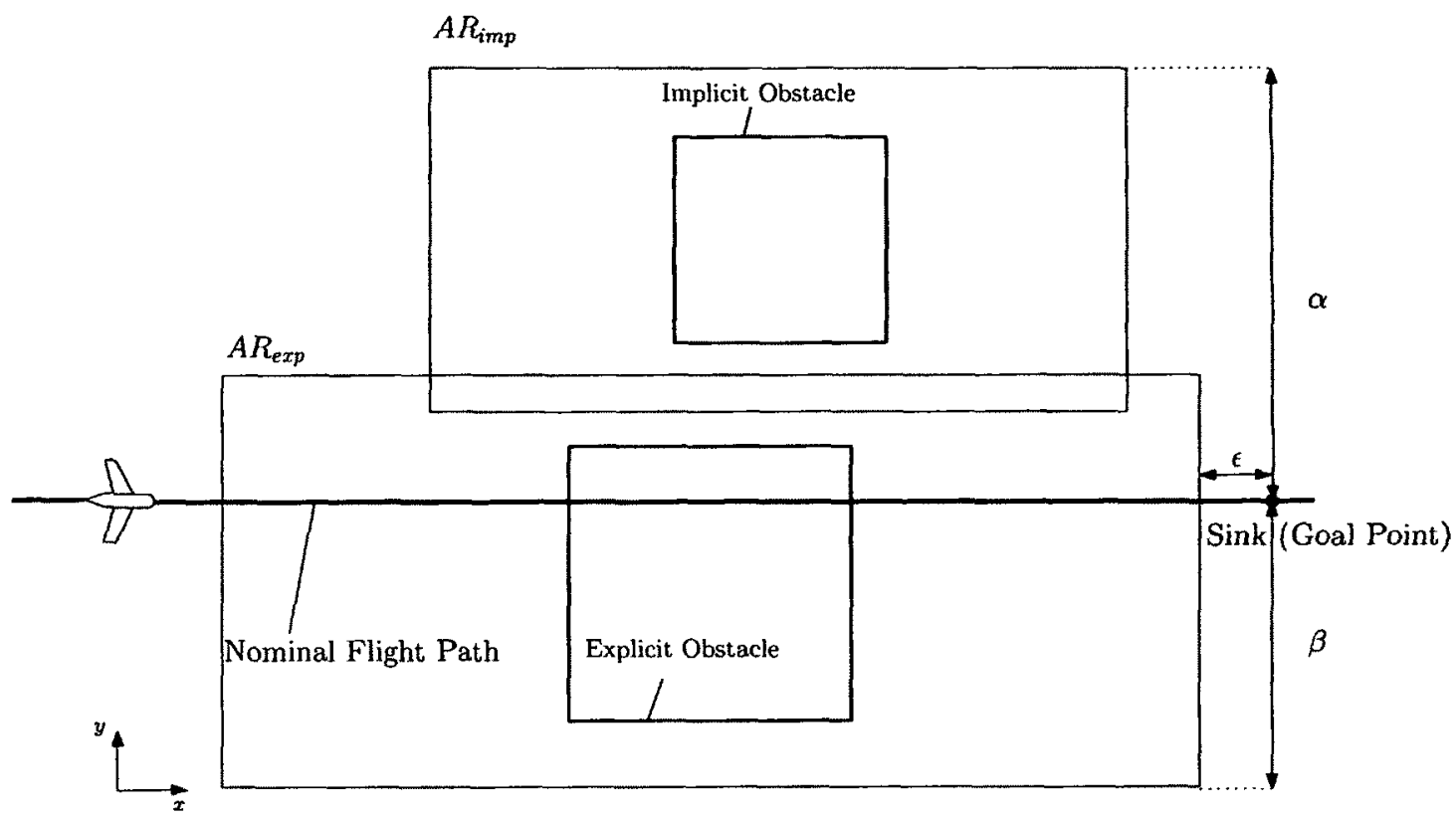

Figure 5.15: Defining $l$, to compute $\Lambda_{g}$

\subsubsection{Calculating the Required Wedge Length, $h$}

In the process of implementing $\mathrm{OA}$, in several instances $h$ is required, e.g. while defining the AR and the AcA.

In order to calculate the required wedge length in the top plane, $h / a$ is interpolated from Figure 3.28 based on $R_{m i n}^{T} / a$. Then

$$
h=\frac{h}{a} \times a
$$

Similarly in the side plane, the same procedure is used while using $R_{\min }^{P}$ and $H$ instead of $R_{\min }^{T}$ and $a$, respectively.

\subsection{Obstacle Avoidance Results}

In this section, the six degree of freedom Aerosonde model is used to test the OA scheme developed in this thesis. The Aerosonde is subjected to multiple obstacle 


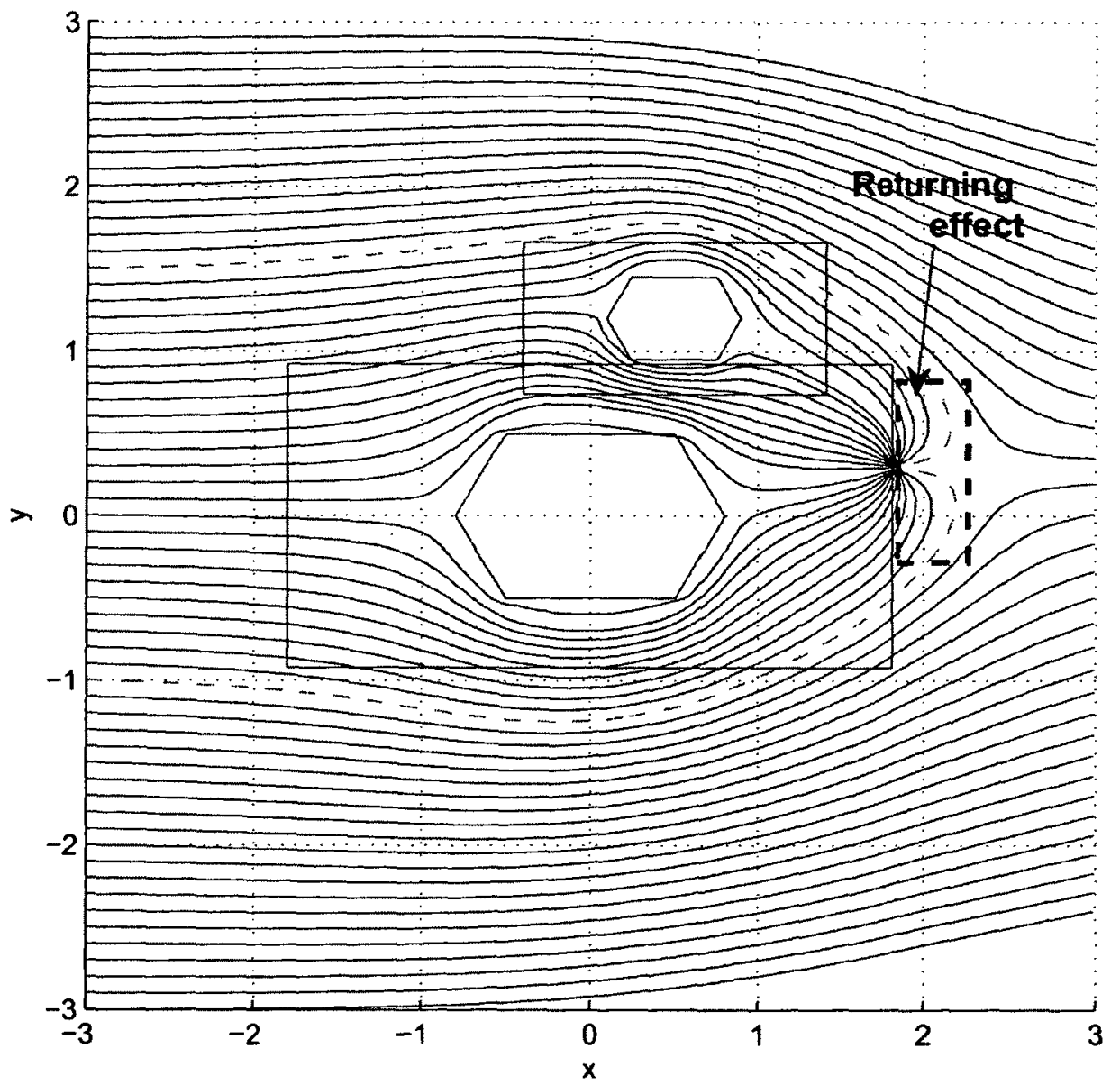

Figure 5.16: The returning effect caused by a sink 
scenarios and the aircraft's response is assessed. The resulting avoidance maneuvers are discussed in detail.

The Aerosonde is set to fly at a cruise speed of $23 \mathrm{~m} / \mathrm{s}$ (from Table 4.1 the Aerosonde's cruise speed is $51 \mathrm{mph} \approx 23 \mathrm{~m} / \mathrm{s}$ ). Furthermore, its bank angle is limited to 60 degrees. Note that for the cruise speed mentioned, the aircraft was tested and was able to maintain altitude while keeping a 60 degree bank angle. Therefore, from $(3.62)$

$$
n=2
$$

and from (3.59) the minimum turning radius is

$$
R_{\min }^{T}=31.13 m
$$

and from (3.64), the minimum pull-up radius is

$$
R_{\min }^{P}=53.92 m
$$

Note that all obstacle scenarios introduced from here on are assumed to have been already magnified according to (5.1), (5.3) and (5.4).

\subsubsection{Fly-around Maneuver}

The aircraft encounters a single obstacle of height $H=75 \mathrm{~m}$ and width $a=50 \mathrm{~m}$. Note that since there is only one obstacle

$$
a_{\text {top }}=a_{\text {side }}=a
$$

The scenario is tested for three different values of $\delta_{y}=20 \mathrm{~m}, 10 \mathrm{~m}, 0 \mathrm{~m}$. The aircraft flies at $50 \mathrm{~m}$ AGL, and is set to maintain this altitude. Therefore, $\delta_{z}=25 \mathrm{~m}$, and from 
(5.7), the Deliberator will chose to perform a fly-around maneuver in all three cases. Figures 5.17 to 5.19 , illustrate the $\mathrm{MO}$, the AR, the goal point and the trajectory followed by the UAV. In these figures, the bold black line is the actual trajectory of the aircraft, whereas the blue line is a pathline whose starting point is the point the aircraft enters the AcA. Finally, the goal point is marked with a square. Note that upon crossing the Deactivation line, the Selector deactivates OA and the aircraft is simply commanded to follow a course of $z^{2} \mathrm{ero}^{3}$.

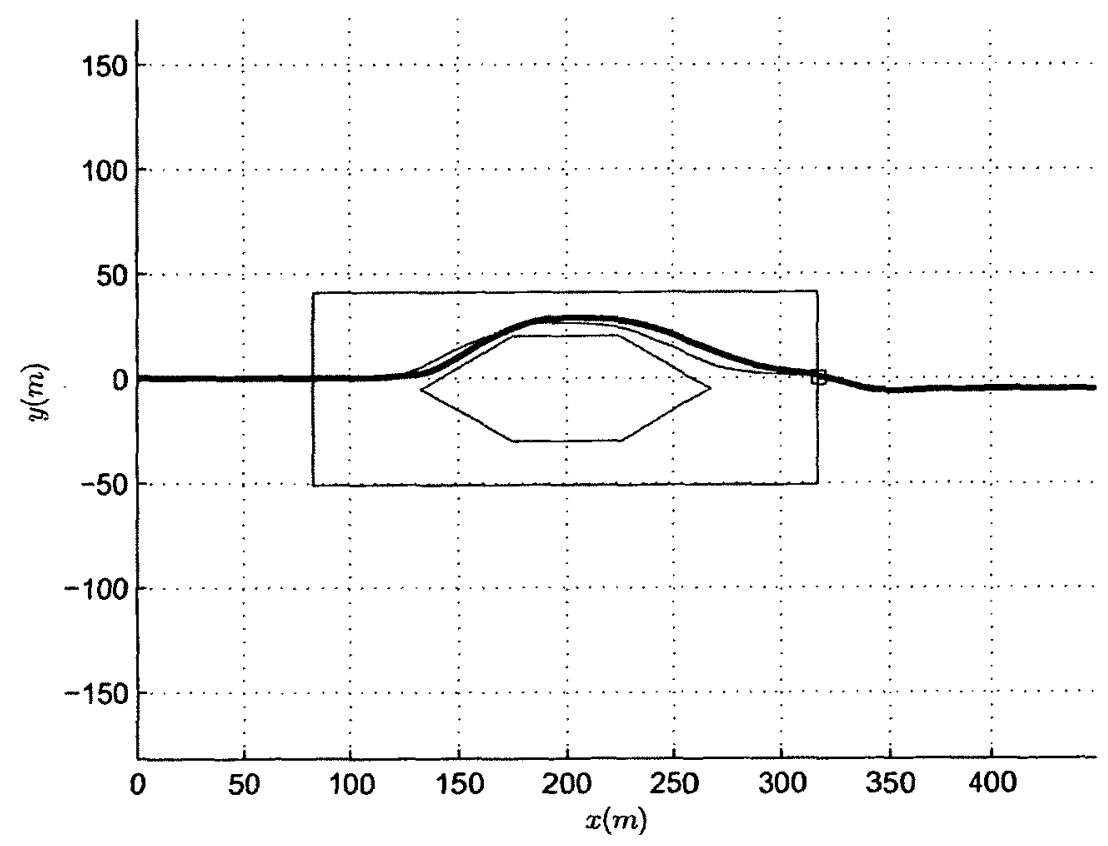

Figure 5.17: Fly-around maneuver for an obstacle of length $50 \mathrm{~m}$, where $\delta_{y}=20 \mathrm{~m}$

It can be seen that the aircraft trajectory has some minor deviations from its corresponding pathline. This occurs because there is a delay between when the.course computed from OA is commanded and when the actual course reaches that value. In an ideal but unrealistic situation where the aircraft's response to a command would

\footnotetext{
${ }^{3}$ If the MicroPilot Autopilot would be used, the aircraft would be commanded to reduce crosstrack error using the controller of Figure 4.6. As such, the crosstrack error observed in Figure 5.17 would not occur.
} 


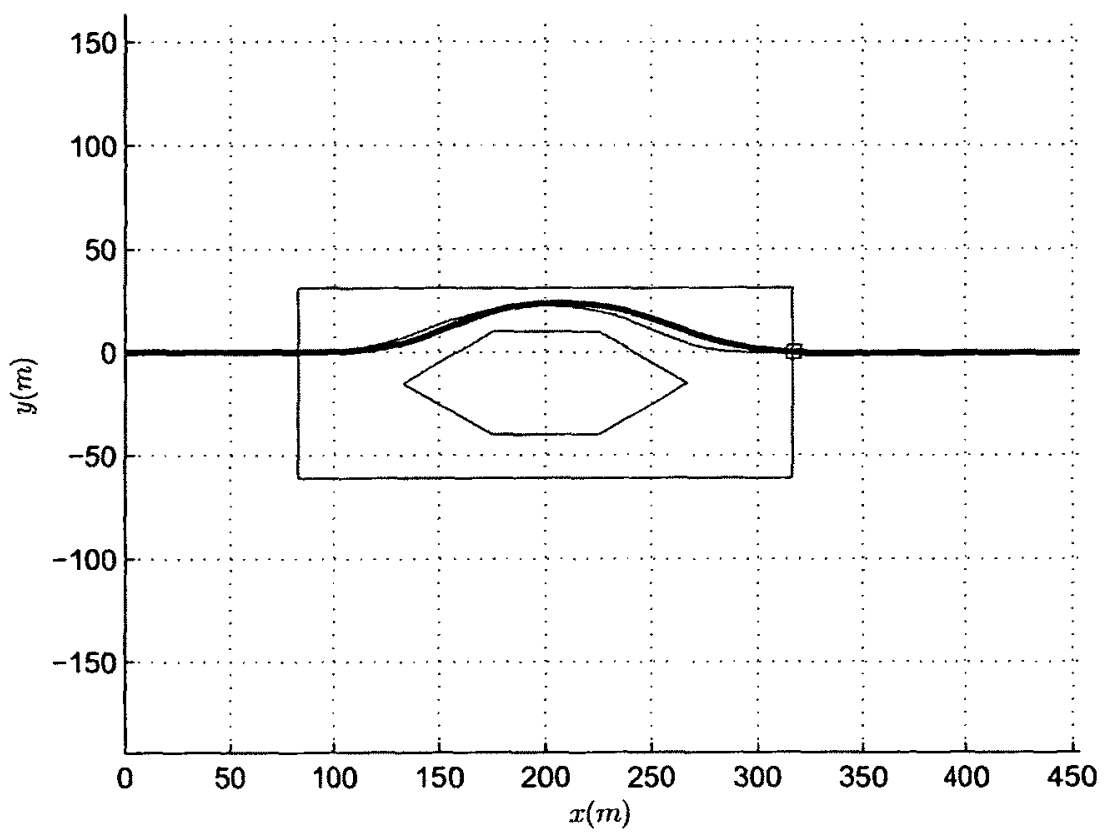

Figure 5.18: Fly-around maneuver for an obstacle of length $50 \mathrm{~m}$, where $\delta_{y}=10 \mathrm{~m}$

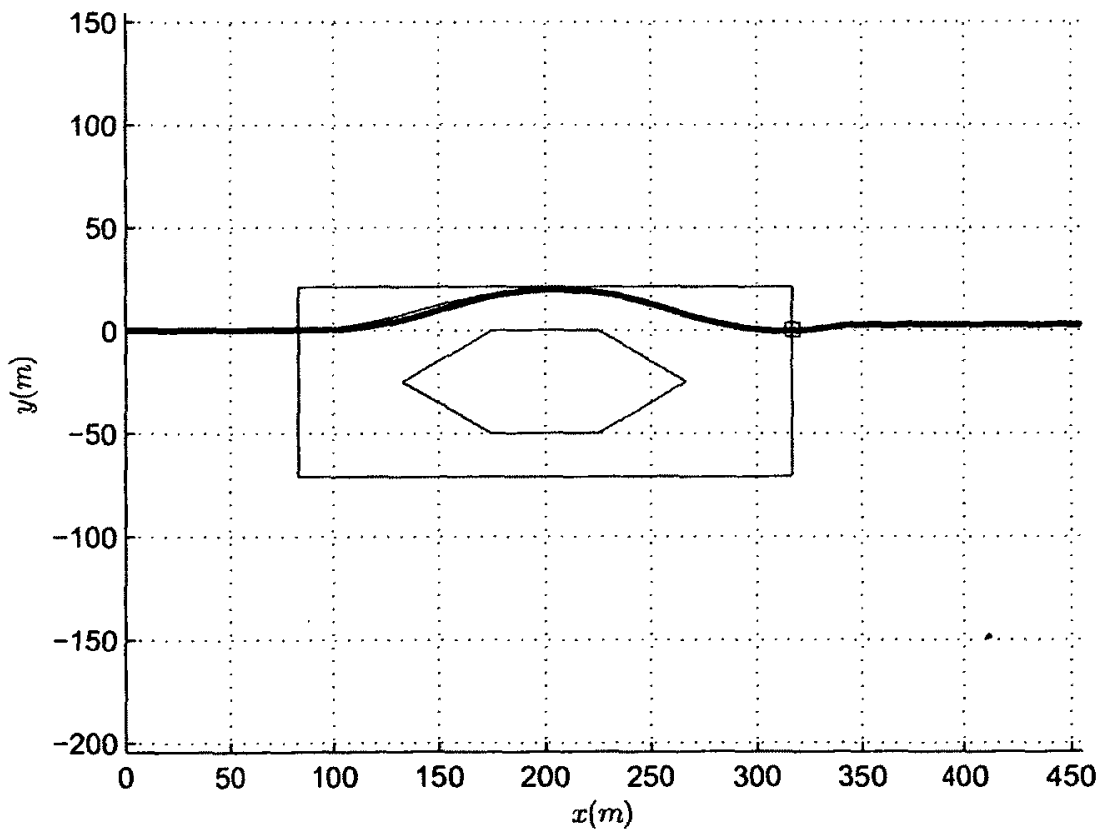

Figure 5.19: Fly-around maneuver for an obstacle of length $50 \mathrm{~m}$, where $\delta_{y}=0 \mathrm{~m}$ 
be instantaneous, the aircraft trajectory and pathline would coincide. Note that for lower values of $\delta_{y}$, the aircraft's trajectory is closer to its corresponding pathline. This happens because for lower values of $\delta_{y}$, lower turning rates are commanded and therefore the aircraft can better follow its command.

In Figure 3.15, a scenario was shown where an obstacle's depth was larger than its width. However, its real depth only became available after the avoidance maneuver had started. In Figure 5.20, this scenario is tested where equal depth and width is initially assumed such as in Figure 5.17. However, at $x=200 \mathrm{~m}$, the obstacle's real depth of $2 a=100 \mathrm{~m}$ is discovered and the velocity field is recalculated. Note that even

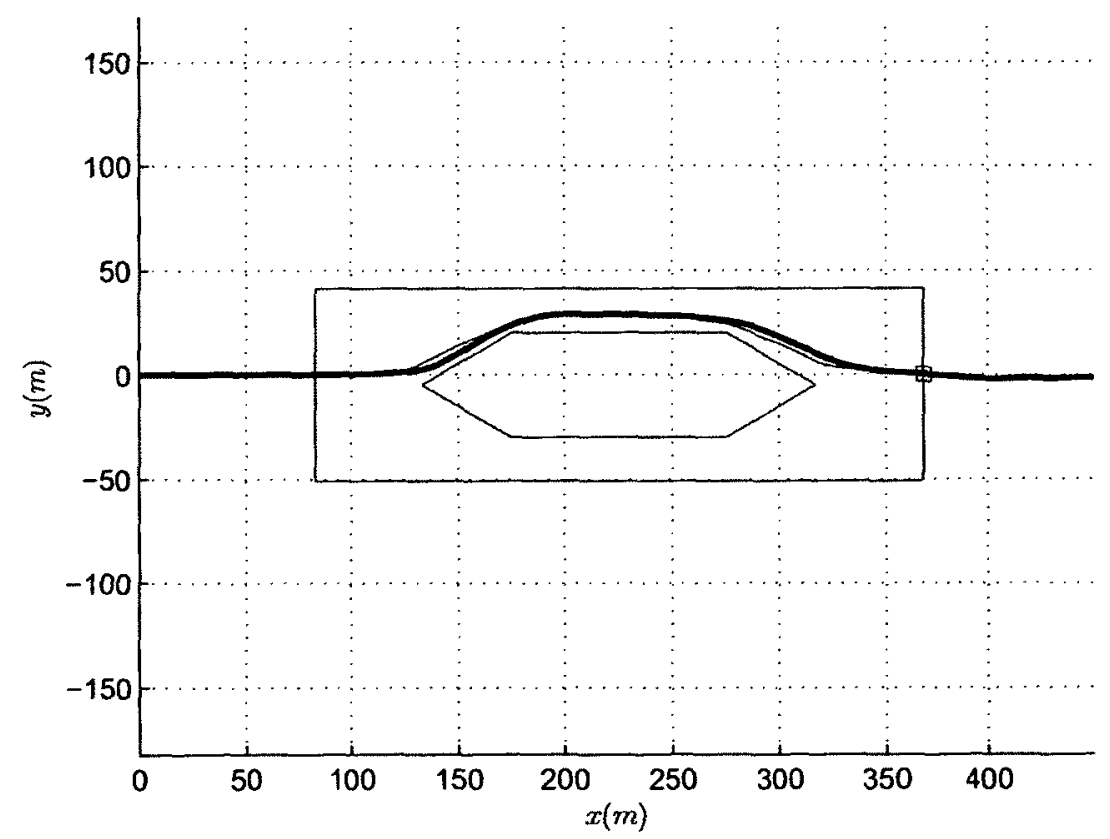

Figure 5.20: Avoidance maneuver with updated obstacle depth while performing the avoidance maneuver

though the obstacles dimension changes while an avoiding maneuver is in process, the avoiding maneuver corrects itself and successfully avoids the obstacle. 


\subsubsection{Fly-over Maneuver}

The same scenario of Figure 5.17, i.e. $\delta_{y}=20 \mathrm{~m}, H=75 \mathrm{~m}$ and $a=50 \mathrm{~m}$, is repeated for three flight altitudes: $60 \mathrm{~m}, 67.5 \mathrm{~m}$ and $70 \mathrm{~m}$ AGL. According to (5.7), the Deliberator will command a fly-over maneuver. In Figures 5.21 to 5.23 the avoidance maneuvers are shown. Note that the side view of the obstacle are shown in gray.

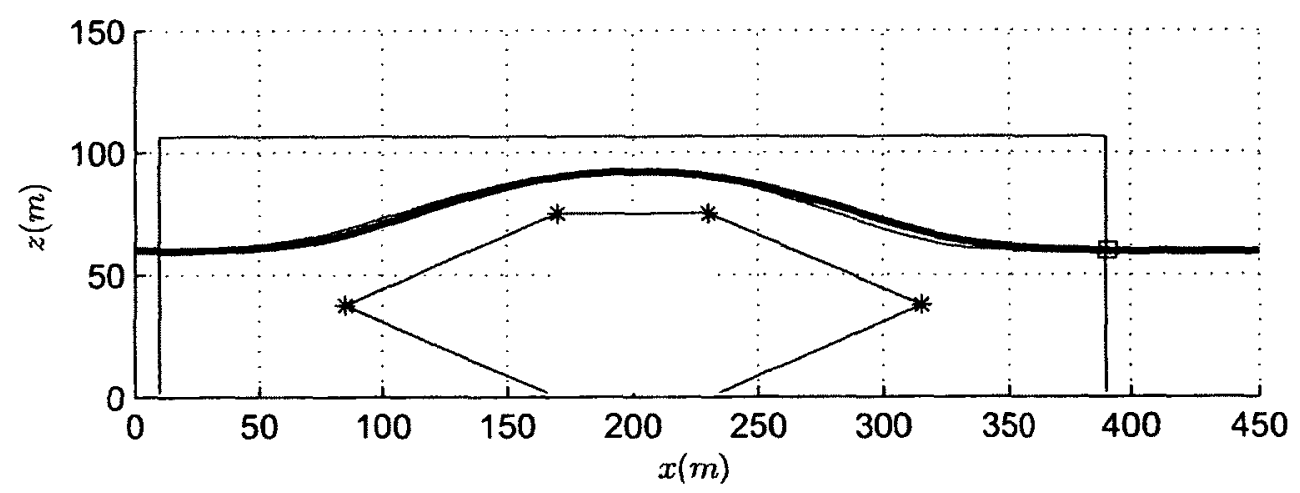

Figure 5.21: Fly-over maneuver for an obstacle of $75 \mathrm{~m}$ height, where $\delta_{z}=15 \mathrm{~m}$

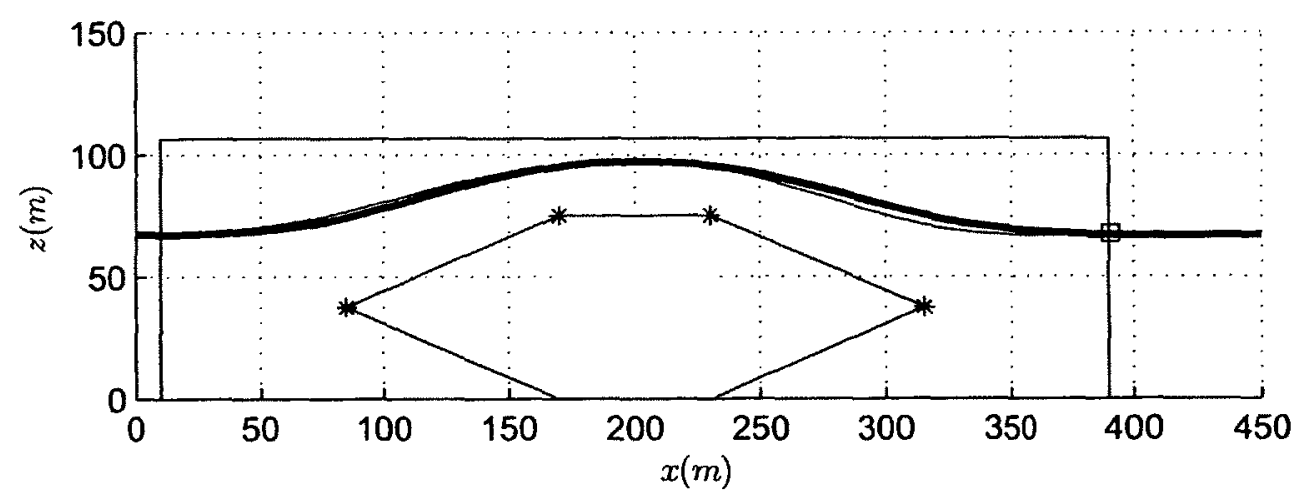

Figure 5.22: Fly-over maneuver for an obstacle of $75 \mathrm{~m}$ height, where $\delta_{z}=7.5 \mathrm{~m}$

The trend seen here is very similar to that of the fly-around maneuvers.

In Figure 5.24, the aircraft is flown at an altitude of $37.5 \mathrm{~m}$ AGL toward an obstacle with $H=75 m, a=80 m, \delta_{y}=40$. Here again the Deliberator commands a fly-over maneuver. From (5.5) 


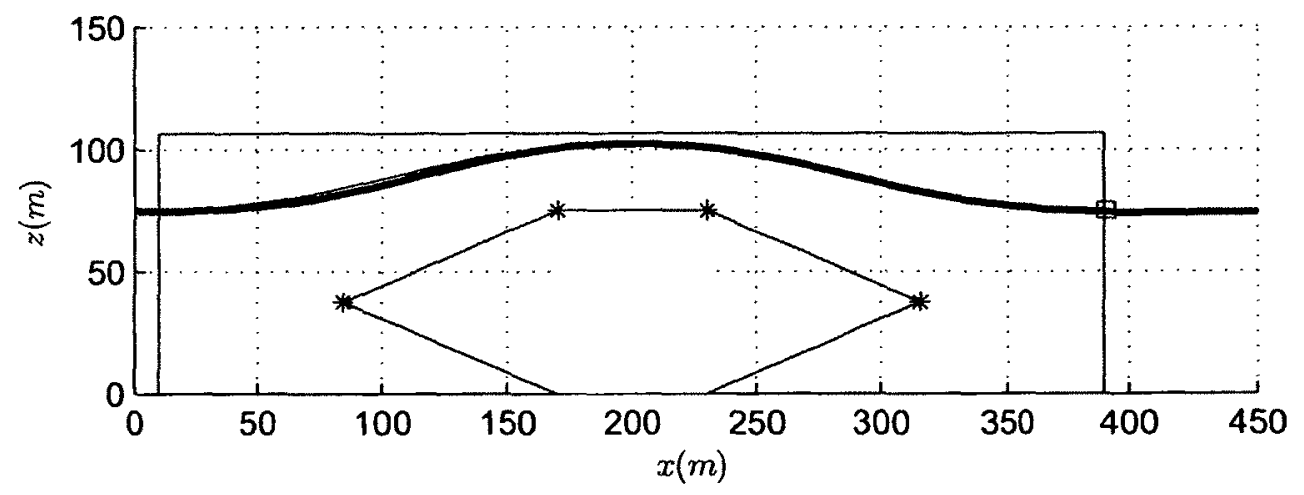

Figure 5.23: Fly-over maneuver for an obstacle of $75 \mathrm{~m}$ height, where $\delta_{z}=0 \mathrm{~m}$

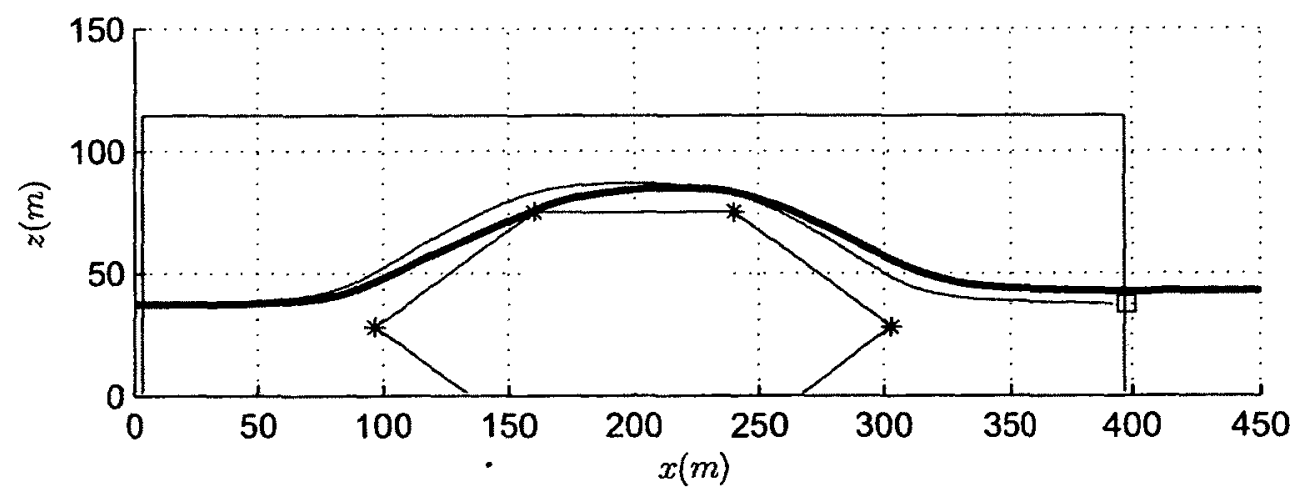

Figure 5.24: Fly-over maneuver for an obstacle of $75 \mathrm{~m}$ height, where $\delta_{z}=37.5 \mathrm{~m}$ 


$$
\delta_{z}=37.5>0.4 H=30
$$

Therefore, from (5.6)

$$
H=93.75 m
$$

In this case, it can be seen that the pull-up maneuver requires a path angle that the aircraft cannot achieve. This results in a larger difference between the avoidance maneuver and the pathline than previous examples. The reason the aircraft cannot achieve such path angle is that the amount of thrust available is limited. As the aircraft's pitch angle increases, a larger component of thrust needs to be used to counteract weight, hence throttle needs to be increased to a point where the maximum throttle is reached.

To take this limitation into consideration, a maximum path angle can be chosen for the aircraft and if the wedge length computed from minimum pull-up radius violates the maximum path angle, the wedge length can be further increased.

\subsubsection{Multiple Obstacles}

In the event of multiple obstacles, the obstacle consideration subsystem either rejects, combines or simultaneously considers multiple obstacles (Section 5.1.1). The Deliberator, defined in Section 5.1.2, then chooses between a fly-over and fly-around maneuver. Note that the chosen maneuver strongly depends on the aircraft's nominal trajectory with respect to the obstacles.

The scenario illustrated in Figure 5.1 is used, and the avoidance maneuvers are shown for three different nominal flight paths. The dimensions of the obstacles are shown in Figure 5.25.

Case I: In this scenario, illustrated in Figure 5.26, the nominal flight path, which is at $40 \mathrm{~m}$ AGL, only intersects the first obstacle. Therefore, obstacle consideration 


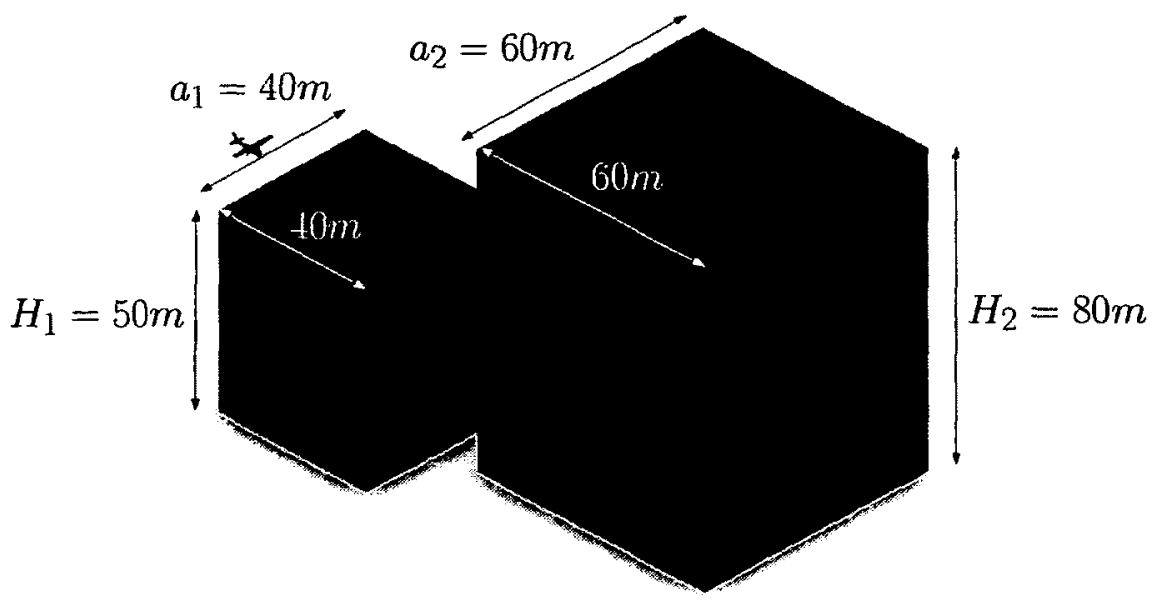

Figure 5.25: Two obstacle scenario - Obstacle dimensioning
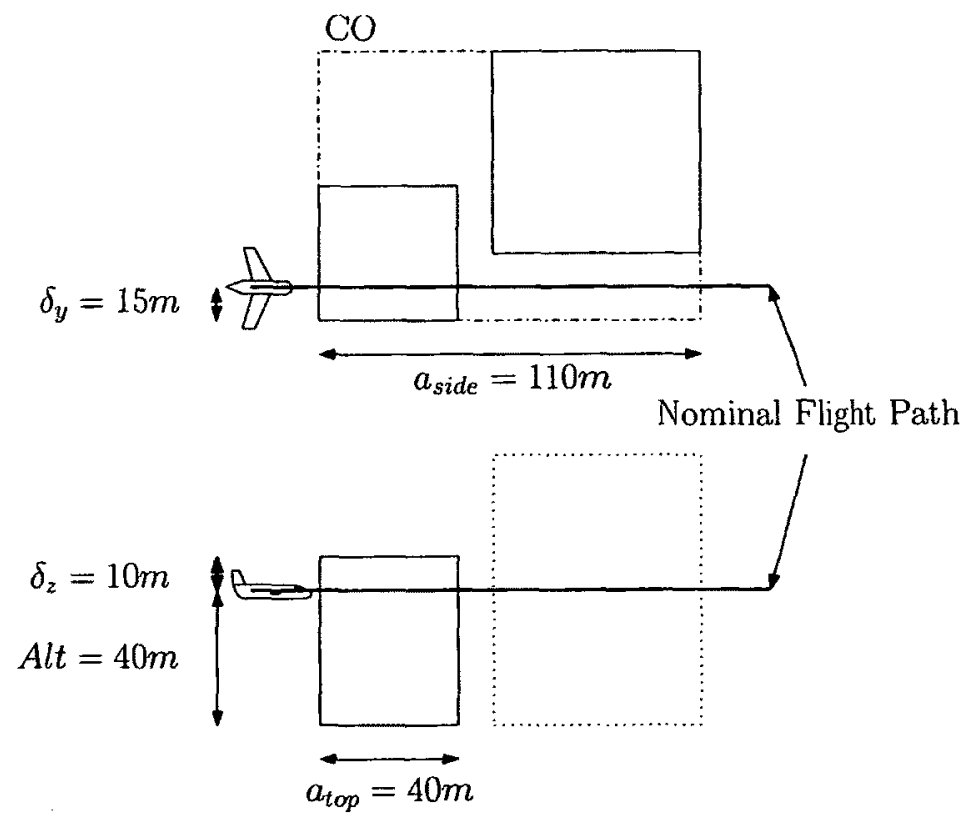

Figure 5.26: Two obstacle scenario - Flight case I 
in the side plane ignores the second obstacle. However, in the top plane, the second obstacle lies in the AR of the first obstacle, hence, the two obstacles are combined. The Deliberator then assesses (5.7):

$$
110+2 \times 15>40+2 \times 10
$$

Therefore a fly-over maneuver is chosen.

Case II: In Figure 5.27, the aircraft is flying at $70 \mathrm{~m}$ AGL and is flying clear of the first obstacle. Therefore, it is ignored in the obstacle consideration of both planes. For this case, (5.7) is

$$
60+2 \times 15>60+2 \times 10
$$

which also leads to commanding a fly-over maneuver.

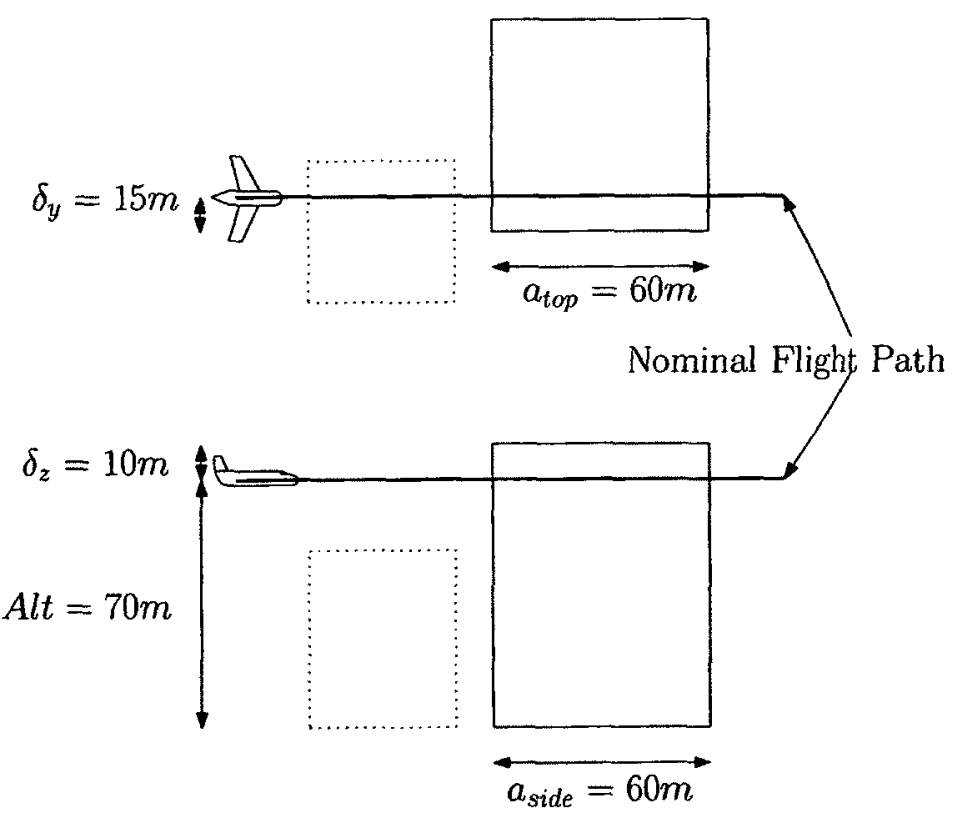

Figure 5.27: Two obstacle scenario - Flight Case II 
Case III: In Figure 5.28, the aircraft's flight path ( $40 \mathrm{~m}$ AGL) intersects both obstacles in both planes and therefore, the two obstacles are combined in both planes. Here, (5.7) is

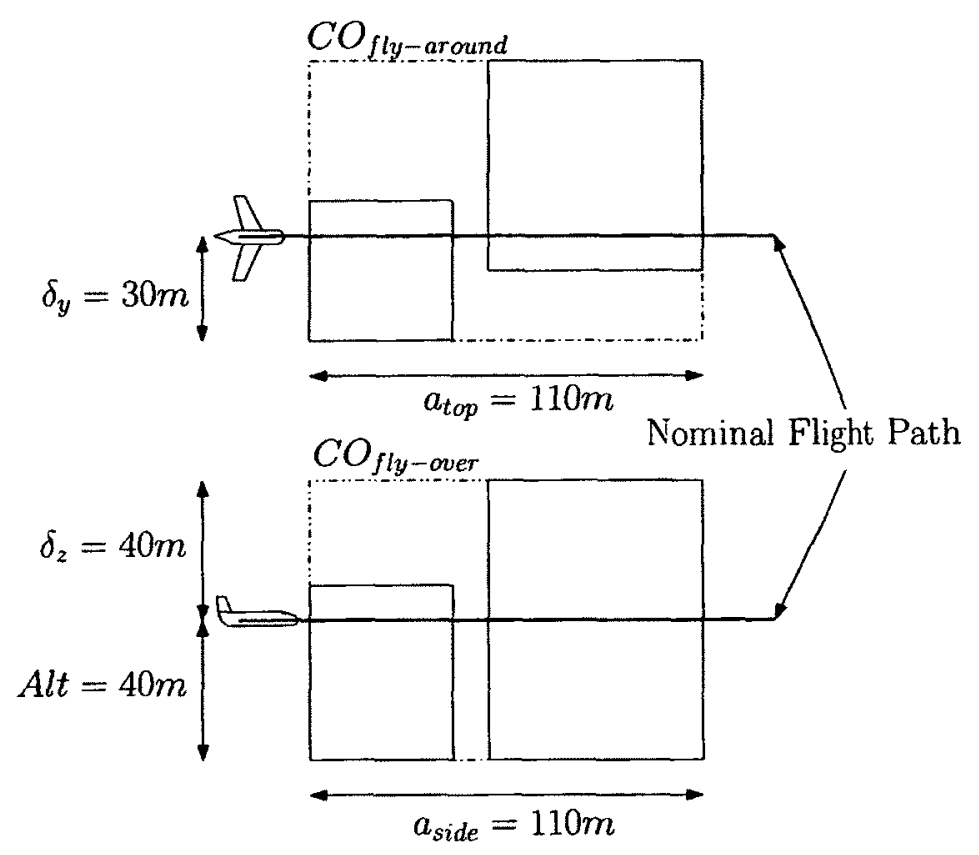

Figure 5.28: Two obstacle scenario - Flight case III

$$
110+2 \times 30<110+2 \times 40
$$

As a result, a fly-around maneuver is commanded. Note that if a fly-over maneuver would have been chosen by the Deliberator, the obstacle would have to be resized because $\delta_{z}>0.4 H$

In Figure 5.29, the trajectory flown by the aircraft when encountering the obstacles is shown for all three cases.

\subsection{Summary}

In the first section of this chapter, the subsystems of the OA hierarchy were defined in detail. It was explained that obstacle consideration is performed within the top and 


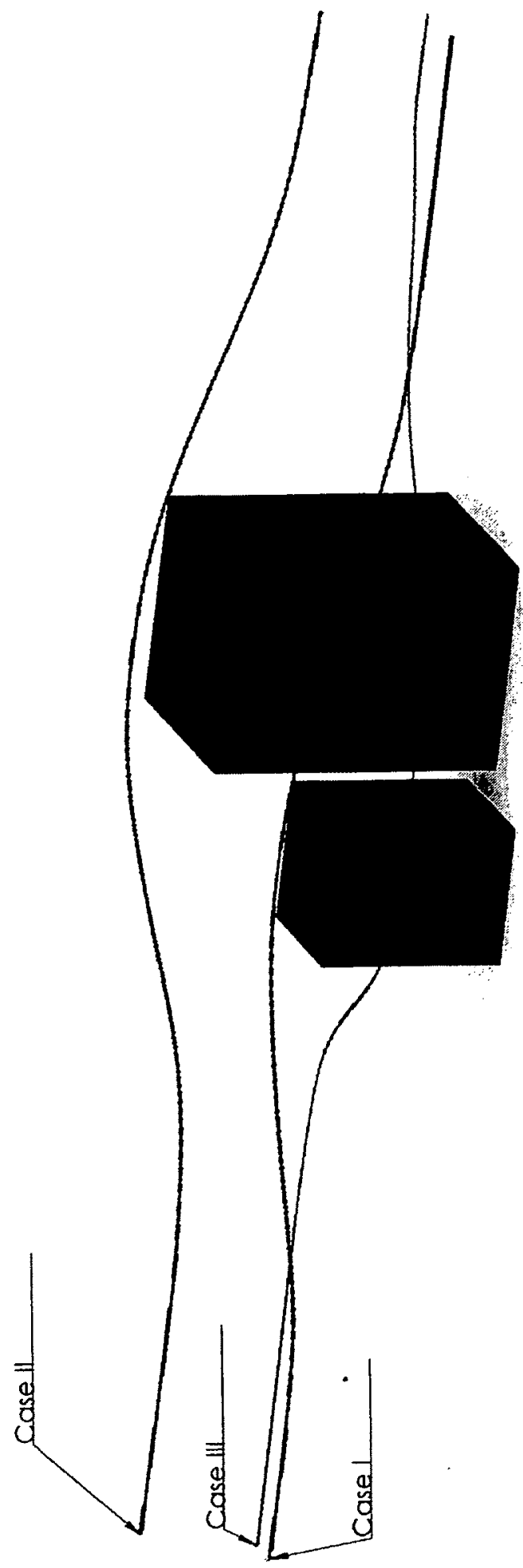

Figure 5.29: OA maneuvers in a scenario with two obstacles for three different cases. 
side plane. In a scenario where more than one obstacle is present, a decision needs to be made: obstacles might be ignored, combined or simultaneously considered. An AR was defined and based on the intersection of the obstacles and ARs this decision was made. Moreover, it was shown that the nominal flight path of the aircraft affects the CO. Afterwards, it was explained that the Deliberator chooses between a flyover and fly-around maneuver based on data obtained from obstacle consideration. Also, wedges were added to the front and back of the $\mathrm{CO}(\mathrm{s})$ to incorporate the aircraft's minimum turning and pull-up radii. Then, the velocity field around the considered obstacle was computed and employed to guide the aircraft during the evasive maneuver. Finally, an AcA was defined to choose when to engage from normal flight to OA.

In the second part of the chapter, various scenarios were flown with the Aerosonde model. It was shown that the Aerosonde successfully avoids obstacles and that the evasive maneuvers are not such that the aircraft is incapable of flying. However, it was noticed that fly-over maneuvers may be harsher than the aircraft can perform. 


\section{Chapter 6}

\section{Autonomous Aircraft Test Bed}

As part of this research, an aircraft test bed was developed to experimentally test OA algorithms. The test bed was developed by integrating enhanced avionics into an off-the-shelf RC model aircraft. The enhanced avionics consist of a commercial autopilot and telemetry.

It should be noted that the obstacle avoidance algorithms from the previous chapters were not implemented on the test bed, and that this chapter only details the test bed development for the benefit of future work.

\subsection{ATB 10}

Within the GeoSurv II project, a line of Avionics Test Beds (ATBs) were developed in the past by undergraduate students to assess the performance of various avionics components. Therefore, the autonomous test bed developed within this research inherited the ATB naming system and was named as ATB 10.

\subsubsection{Senior Telemaster}

The model aircraft used for the test bed is a Senior Telemaster, shown in Figure 6.1. The Telemaster is a high-wing tail dragger with a wingspan of $2.41 \mathrm{~m}$ (95") and 


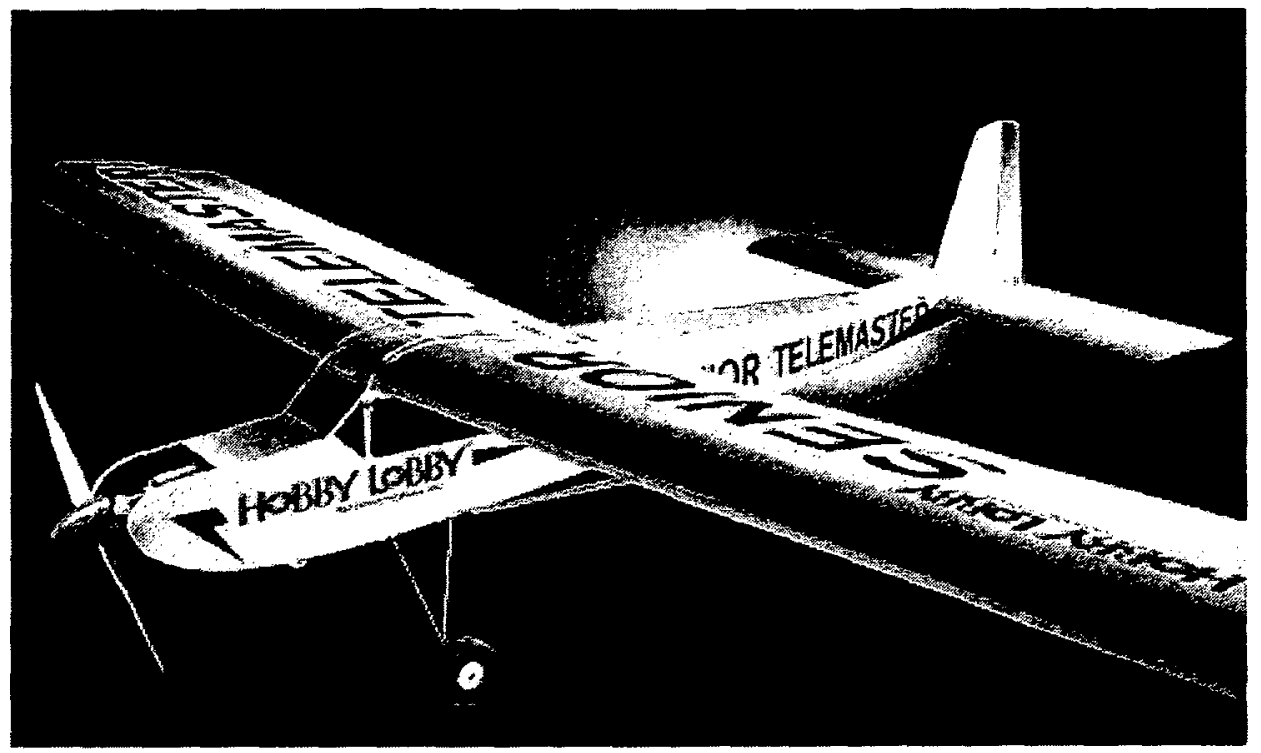

Figure 6.1: The Senior Telemaster

a length of $1.60 \mathrm{~m}\left(63^{\prime \prime}\right)$. The model has three sets of control surfaces: ailerons, elevators and a rudder. For propulsion, the aircraft is fitted with an electric motor that is powered by a $5000 \mathrm{mAh}$ Li-Po battery. With such a battery, the test bed is able to fly for over 20 minutes $^{1}$. A nine channel $2.4 \mathrm{GHz}$ transmitter and receiver, shown in Figure 6.2, is used to transmit pilot commands to the actuators of the aircraft.

The senior Telemaster was chosen for its large load factor, and low cruise speed. These characteristics made the aircraft easier to control, and therefore a suitable test bed candidate for autonomy research. Furthermore, due to its large load factor, the test bed could carry a large payload consisting of additional equipment.

\subsubsection{Autopilot}

In Chapter 5, it was shown that the OA method computes a course/path angle command that guides the UAV to avoid obstacles. However, in order to convert these

\footnotetext{
'The actual flight time depends on the "amount of throttle" used.
} 


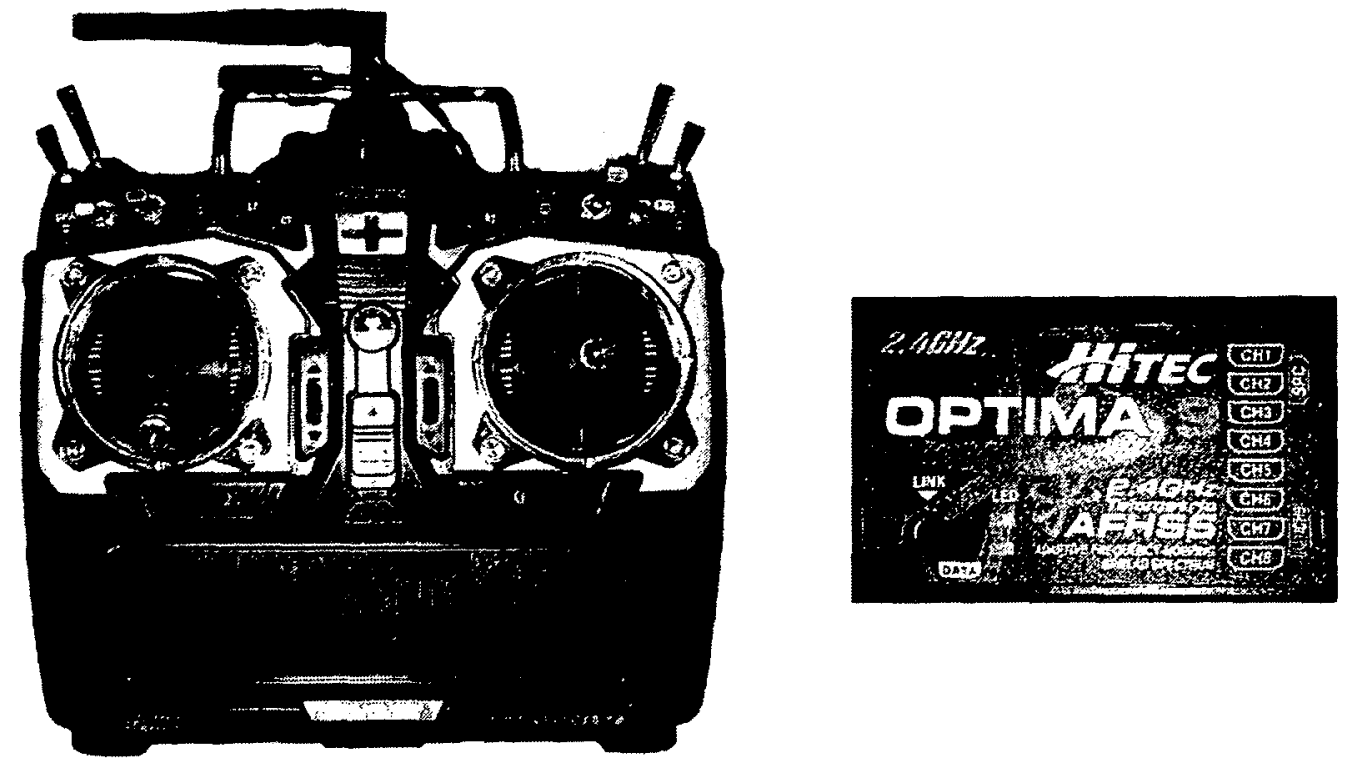

Figure 6.2: The transmitter (left) and receiver (right)

commands to control surface deflections an autopilot is required. The autopilot used on ATB 10 is an off-the-shelf MP2028 autopilot manufactured by MicroPilot ${ }^{\circledR}$. The MP2028 control architecture is very similar to that of described in Section 4.2.1. After tuning the autopilot, it can guide an aircraft through WPs for autonomous flight. All required sensors such as GPS, accelerometers and gyroscopes are embedded on the autopilot. The MP2028, shown in Figure 6.3, weighs $28 \mathrm{gr}$ and measures $10 \mathrm{~cm}$ by $4 \mathrm{~cm}$.

The operator can choose between two flight modes: Pilot In Command (PIC) and Computer In Control (CIC). In PIC mode, the commands received from the transmitter are used to guide the aircraft. In contrast, when the autopilot is switched to CIC mode, the autopilot performs the flight mission that is has been commanded and commands received from the transmitter are ignored. The flight mode is chosen by the operator and gives the operator the ability to engage and disengage the autopilot at will. For instance, the operator might want to take off and land manually due to bad weather. Furthermore, in the case of an emergency the operator can instantly 


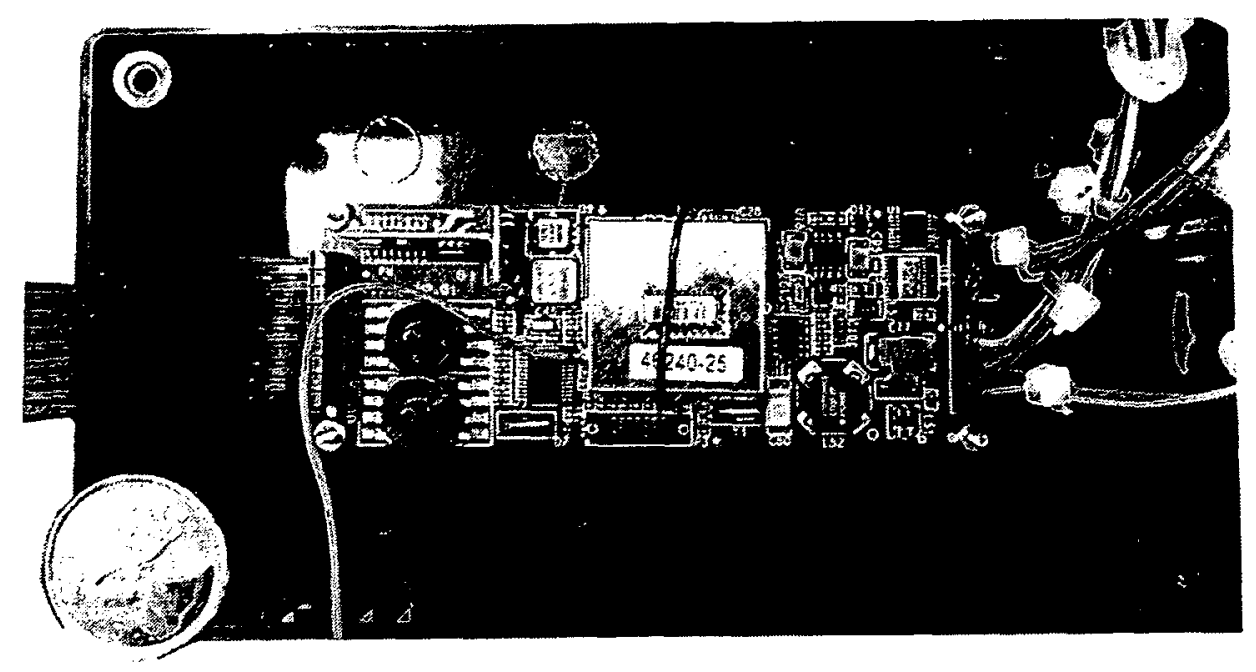

Figure 6.3: The MP2028 autopilot

take over the flight controls.

A Ground Control Software (GCS) is provided with the MP2028 called Horizon. A snapshot of the user-interface of Horizon is shown in Figure 6.4. Horizon allows the operator to monitor the UAV's flight and to change parameters such as controller gains and flight commands. The communication between the autopilot and GCS is established through a standard serial port. If telemetry is available, the parameters mentioned above can be changed in flight.

\subsubsection{Telemetry}

The telemetry used for ATB 10 was a pair of MicroHard ${ }^{\circledR}$ n920-ENC radio modems. The radio modems, shown in Figure 6.5, operate at $900 \mathrm{MHz}$ using FHSS $^{2}$ and are claimed to have a range of over $100 \mathrm{~km}$. Furthermore, they weigh $220 \mathrm{gr}$ and are capable of transmitting data wirelessly at 19200-230400 bps.

The overall setup of the ATB 10 and the ground control station is shown in Figure 6.6.

\footnotetext{
${ }^{2}$ Frequency-hopping spread spectrum
} 


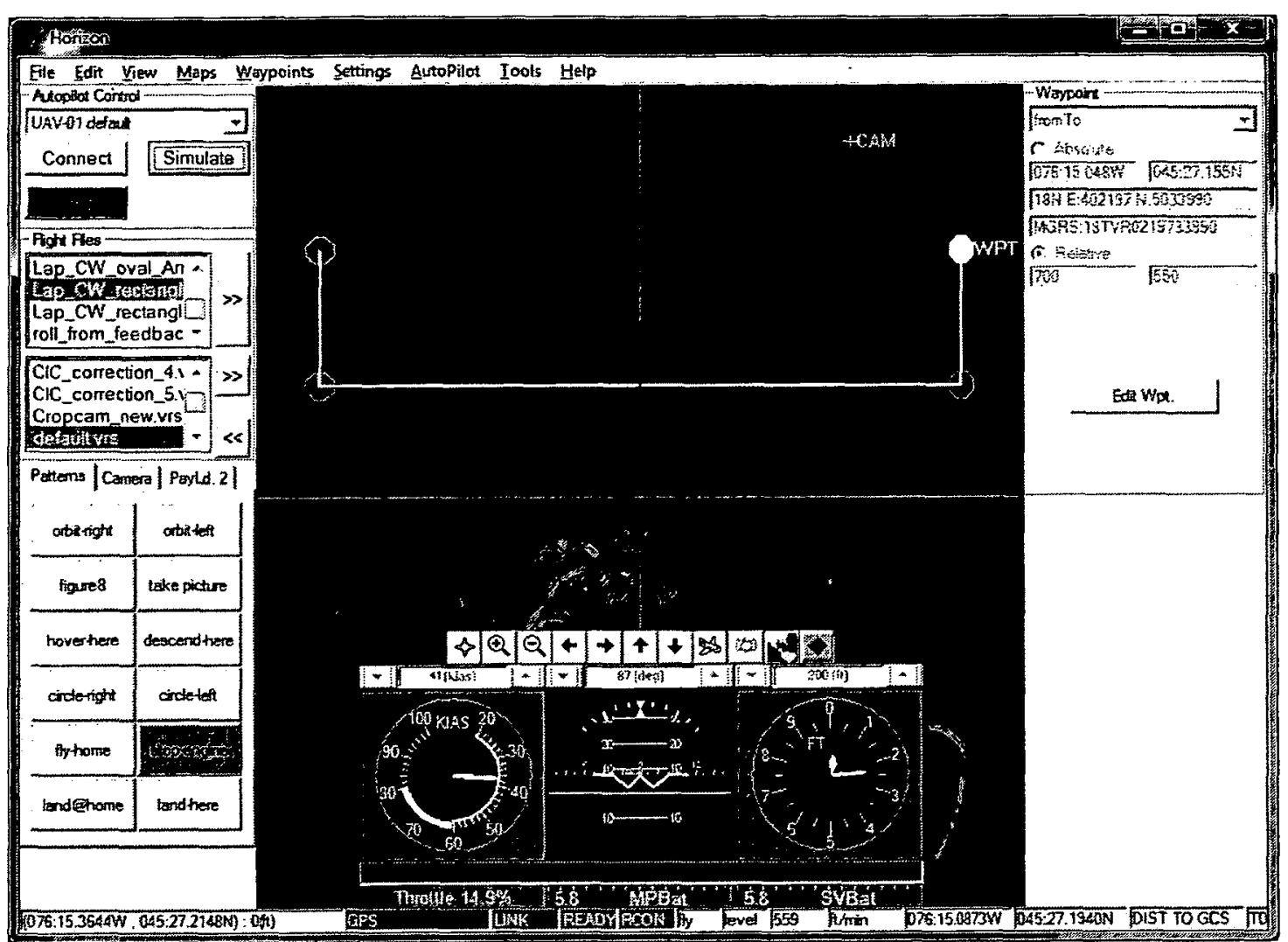

Figure 6.4: Horizon GCS showing ATB 10 in flight

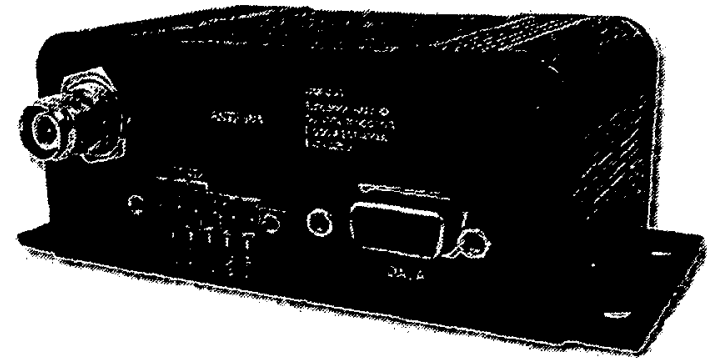

Figure 6.5: MicroHard $^{\circledR}$ n920-ENC radio modem 


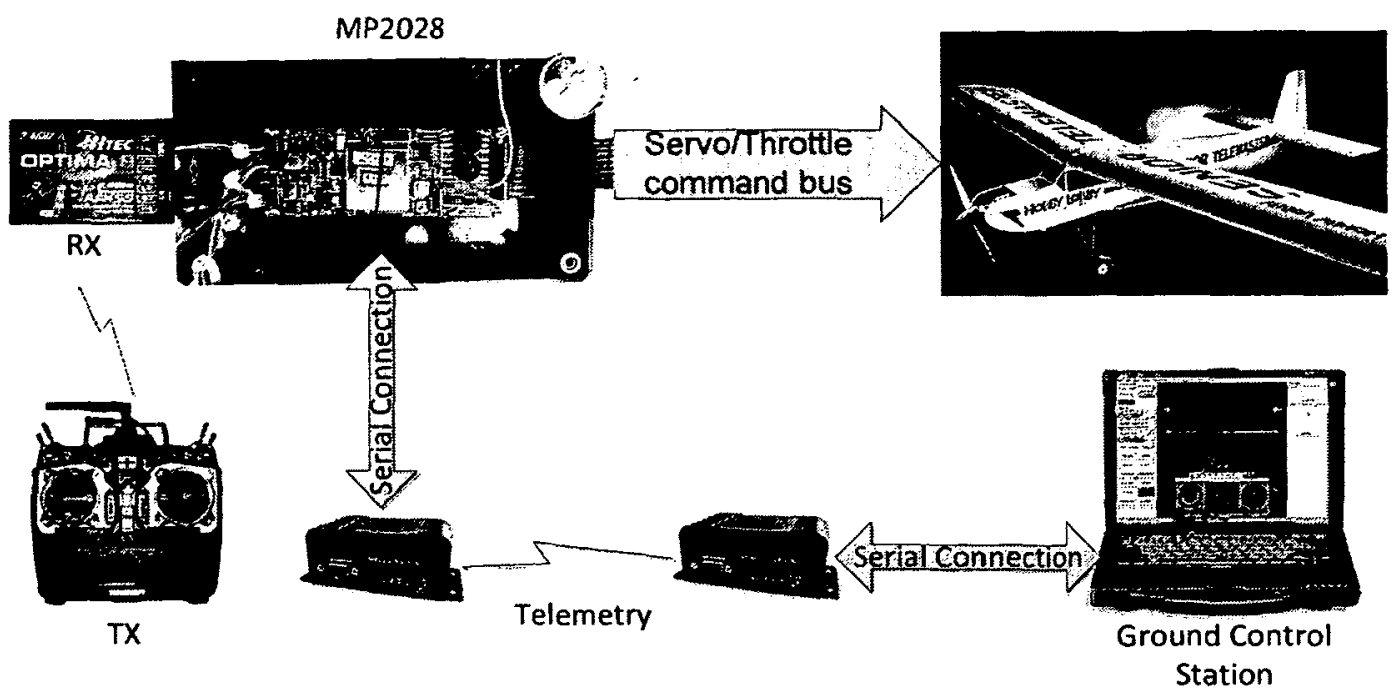

Figure 6.6: ATB 10 and ground control station setup

\subsection{Flight Tests}

The main challenge in integrating the autopilot into the airframe was tuning the PID controllers. This task was a lengthy process that took over 20 hours of flight. During each flight, the aircraft's response and commands were logged for different gain values. Upon landing the data was analyzed and based on the aircraft's responses new gain values were tested and finally chosen.

In Figure 6.7, a test flight is shown where a circuit similar to that of Figure 4.4 was flown for 10 laps during which the controllers were being fine tuned. Note that the two sections of the trajectory highlighted in red define the aircraft being in PIC mode. These segments correspond to manual take-off and landing. The trajectory with the best response is shown in Figure 6.8. Note that the star denotes the location of the ground control station and is set as the origin. Furthermore the positive direction of the vertical axis points toward North. In these flights the wind was in the North direction and therefore the aircraft was blown downwind while performing 


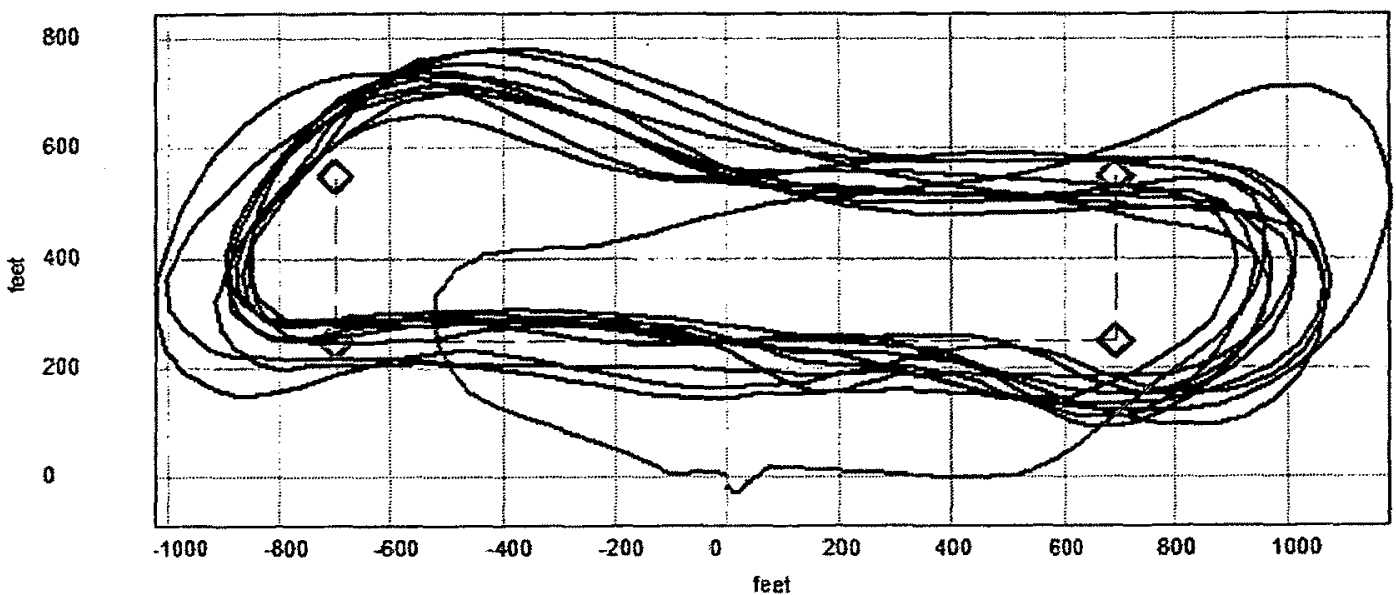

Figure 6.7: Fine-tuning controller gains over 10 laps (laps were flown clockwise)

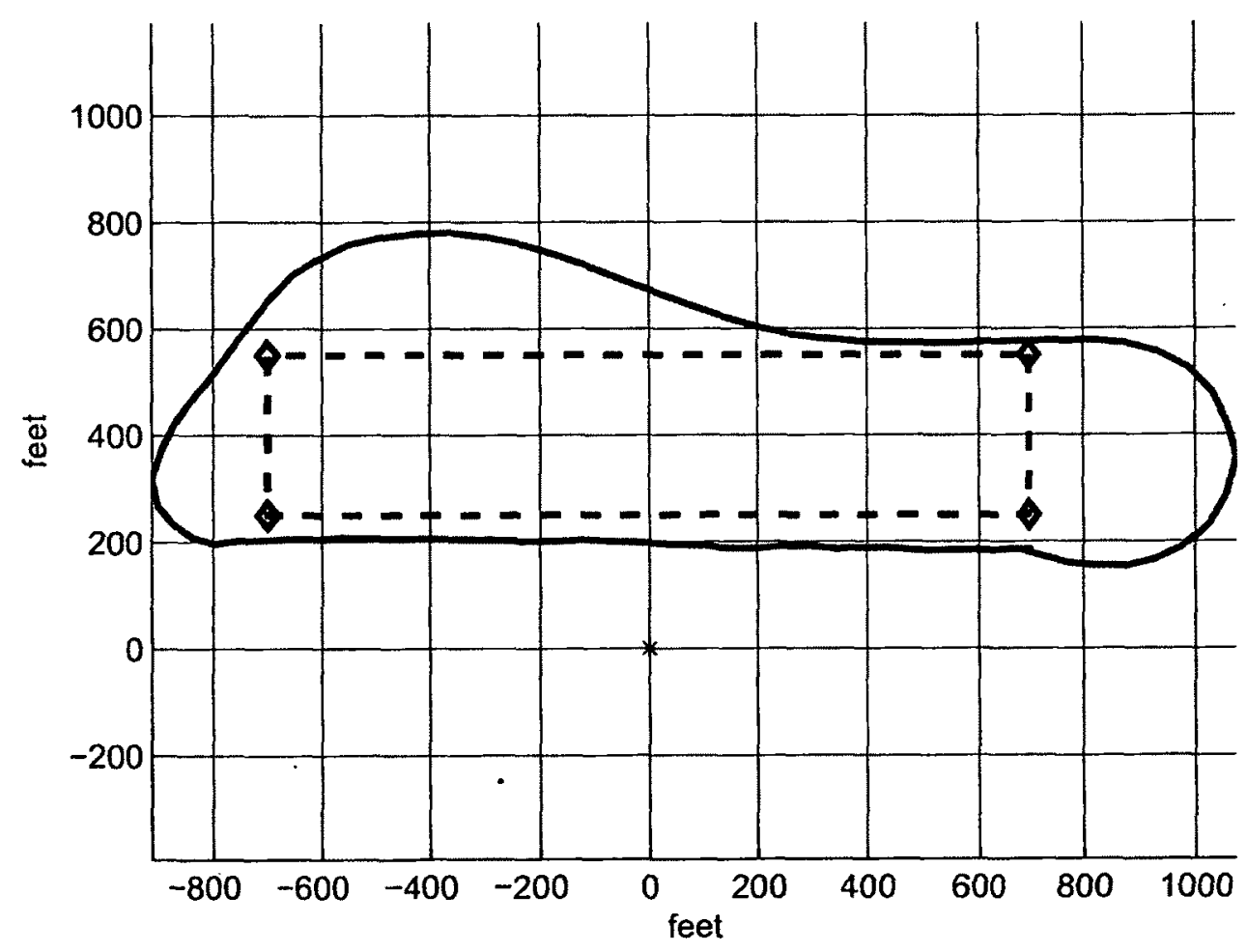

Figure 6.8: Circuit flown with fine-tuned autopilot (lap was flown clockwise) 
the North-western turn.

In Figure 6.9, a survey that involves several passes, similar to that of GeoSurv II, was commanded to ATB 10. During this flight test, the wind was very strong with violent gusts ${ }^{3}$. The effect of the gusts can be seen all along the flight trajectory.

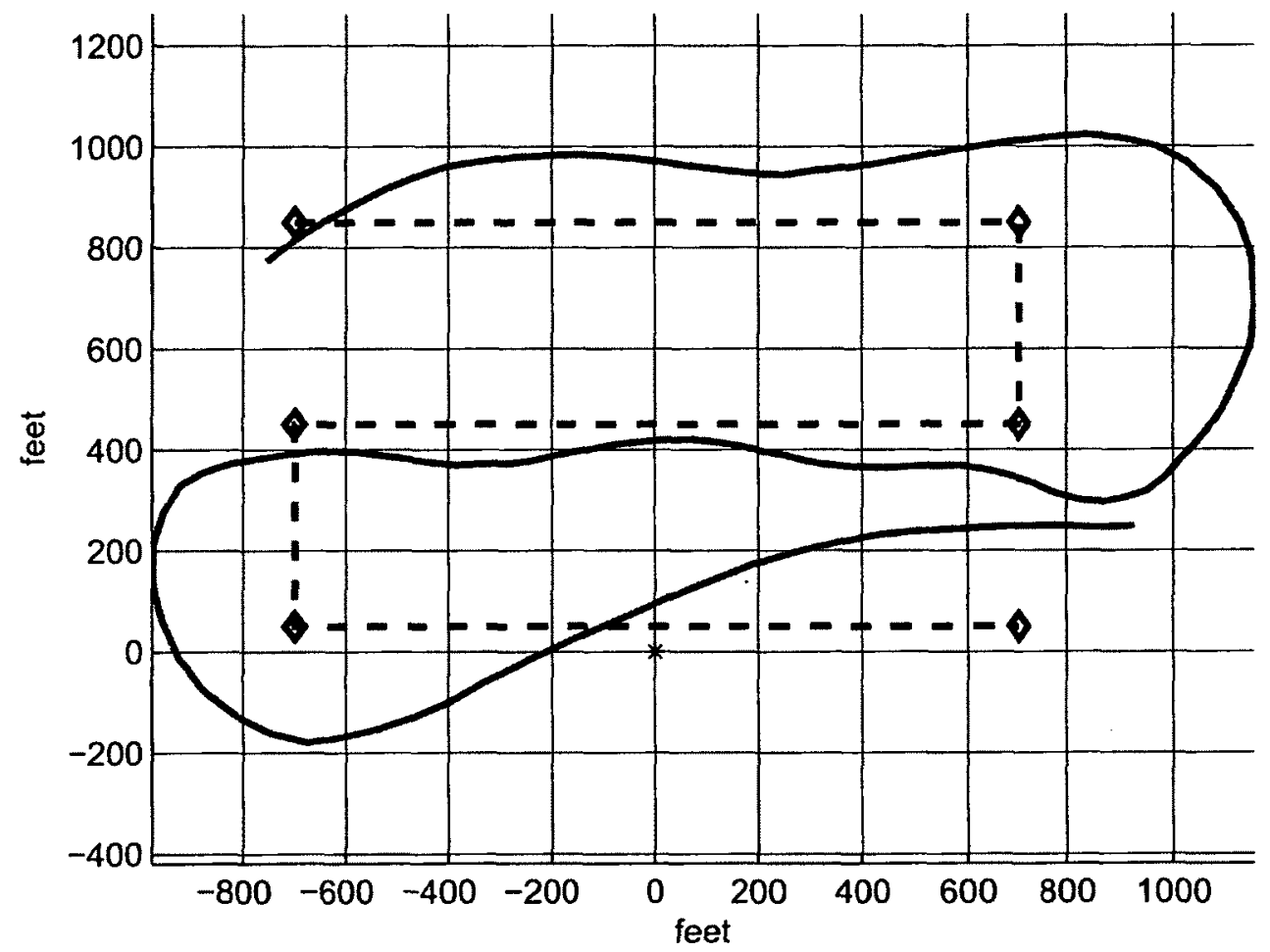

Figure 6.9: Survey flown with fine-tuned autopilot under strong wind with violent gusts

Note that in the test flights, the aircraft was to be kept within visual range so that the pilot could guide the UAV manually if needed. As a result, the aircraft's flight passes were short, limiting the UAV's time to reach the desired pattern.

In Figure 6.10, ATB 10 is shown during autonomous flight.

\footnotetext{
${ }^{3}$ According to the MP2028 manual [51], the UAV should only be flown for wind speeds that are less than half of the aircraft's cruise speed. During this flight this condition was barely met.
} 


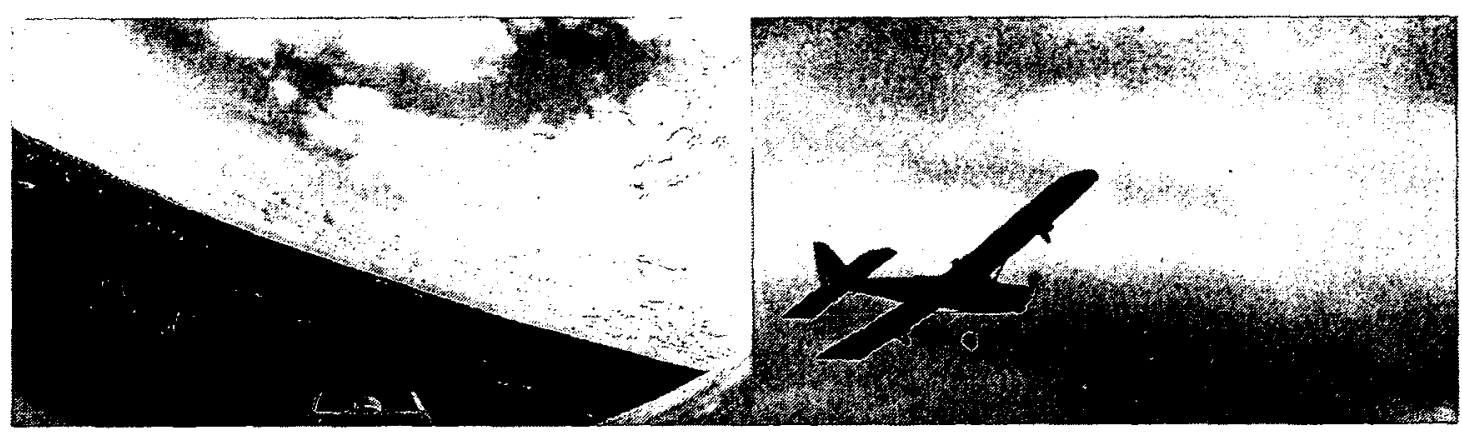

Figure 6.10: ATB 10 during autonomous flight

\subsection{Obstacle Avoidance Flight Test}

For testing, the OA algorithm is to be run at the ground control station. The aircraft is set to fly in an obstacle free environment but an imaginary obstacle is considered on the path of the aircraft. The GPS location of the aircraft is sent to the ground station, which will check when the UAV enters the AcA of the imaginary obstacle. Upon entering, the deliberator which has decided between a fly-around and fly-over maneuver, computes the desired path or course angle. Then, from the error in path/course angle, the desired pitch/heading is computed and commanded to the autopilot. The OA test setup is shown in Figure 6.11.

Due to shortage of time and bad weather, the OA algorithm was not tested on ATB 10 . 


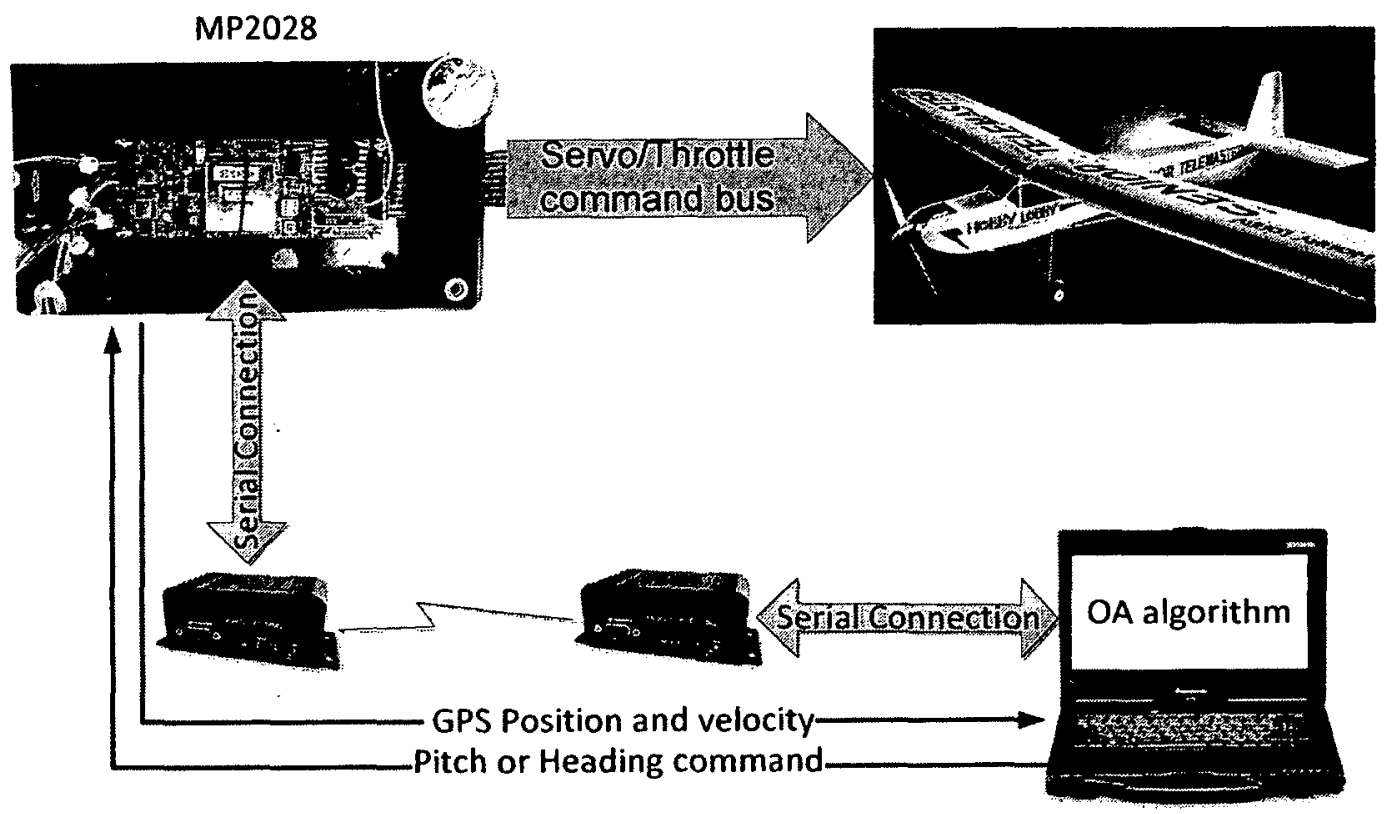

Figure 6.11: Obstacle avoidance test setup 


\section{Chapter 7}

\section{Conclusions}

The objective of this thesis was to develop a Reactive Obstacle Avoidance (ROA) method for a UAV performing geophysical surveys. The method was to consider aircraft constraints and be able to run in real-time with limited computation resources. Furthermore, the method was to be developed for potential obstacles that would be encountered when flying at low altitudes.

The method chosen for ROA was the potential flow (panel) method. Using the panel method, the obstacles were modeled within a uniform flow and the velocity field of such flow was used to guide the UAV. The method was shown to be fast and therefore achievable in real-time with limited computation resources. It was shown that the potential flow method could be used for multiple obstacles and because the potential field corresponding to the flow was harmonic, it was not vulnerable to local minima, a problem well-known in the Artificial Potential Field (APF) method.

The method proposed employed an "appropriate" modelling of obstacles in order to take aircraft constraints into account. This was achieved by adding wedges to the front and back of obstacles, which decreased the curvature of the flow's pathlines. A graph was created that showed the correlation between the wedge size and the minimum curvature of resulting trajectories. This correlation was used to determine the required wedge length based on the aircraft's constraints, i.e. minimum turning and 
pull-up radii. Furthermore, the modelling was done while considering the limitations of obstacle detection. It was shown that as soon as more data about obstacles, such as their actual depth, would become available, the avoidance algorithm would cope with the new data.

In order to test the OA method developed on an aircraft model, the non-linear model of the Aerosonde was used. However, an autopilot had to be integrated to the model for avoidance testing. This was done by using an architecture similar to that used in autopilots manufactured by MicroPilot. In order to be able to implement OA, some modifications were made to the original architecture. The main difference was the addition of two controllers: calculating pitch angle commands from path angle error and calculating heading commands from course error. The addition of these two controllers resulted in the aircraft being accurately guided. Furthermore, these modifications made the aircraft guidable in windy scenarios.

\subsection{Contributions}

- A method for ROA was developed that required low computation resources and had the ability to be run in real-time. The avoidance method took aircraft constraints into account and therefore the evasive maneuvers commanded to the UAV were feasible. In the literature, many of the avoidance methods have been developed for rotorcrafts, which have higher maneuverability than fixedwing aircraft. However, in this thesis the avoidance method was developed for a fixed-wing UAV. Furthermore, UAV Obstacle Avoidance (OA) research has highly been focused on avoiding obstacles encountered in usual flight altitudes, i.e...other aircraft. In contrast, the method developed in this thesis was focused on avoiding obstacles that were encountered in low-altitude flight.

- A test bed was developed to test the OA method developed in this thesis. 
Furthermore, the test bed created could be used for further research in the field of UAV autonomy.

\subsection{Future Work}

There is a large amount of work that can be done within the framework of OA for the GeoSurv II project. A summary of recommendations for future work is given below.

1. Testing the ROA method developed in this thesis on the ATB 10 test bed for imaginary obstacle scenarios.

2. Investigating the hardware required to run OA algorithms on-board of the UAV. The algorithm should then be implemented on the hardware. Then, OA can be performed on board of the UAV for imaginary obstacle scenarios.

3. Combining on-board Obstacle Detection (OD) and OA on ATB 10 and testing avoidance for real obstacle scenarios.

4. Integrating an avoidance method for avoiding other aircraft on ATB 10. Upon integration of such a method, ATB 10 (and potentially GeoSurv II) would have the ability to operate in non-segregated airspace.

5. Ultimately, installing OA hardware on GeoSurv II and testing avoidance scenarios. 


\section{List of References}

[1] J. A. Barnard. "The use of unmanned aircraft in oil, gas and mineral E+P activities." In "Society of Exploration Geophysicists," page 1132. Las Vegas, Nevada (2008).

[2] D. W. Casbeer, S.-M. Li, R. W. Beard, R. K. Mehra, and T. W. McLain. "Forest fire monitoring with multiple small UAVs." In "Proceedings of the 2005 American Control Conference," pages 3530-3535. IEEE (2005).

[3] M. De Biasio, T. Arnold, R. Leitner, and R. Meestert. "UAV based multispectral imaging system for environmental monitoring." In "Proceedings 2011 20th IMEKO TC2 Symposium on Photonics in Measurement (20th ISPM 2011)," pages 69-73. Shaker Verlag GmbH (2011).

[4] A. R. Girard, A. S. Howell, and J. K. Hedrick. "Border patrol and surveillance missions using multiple unmanned air vehicles." In "43rd IEEE Conference on Decision and Control (CDC)," volume 1, pages 620-625. IEEE (2004).

[5] X. Li and L. Yang. "Design and implementation of UAV intelligent aerial photography system BT." In "Proceedings of the 2012 4th International Conference on Intelligent Human-Machine Systems and Cybernetics, IHMSC 2012," volume 2, pages 200-203. IEEE Computer Society (2012).

[6] D. Hausamann, W. Zirnig, G. Schreier, and P. Strobl. "Monitoring of gas pipelines - a civil UAV application." Aircraft Engineering and Aerospace Technology 77(5), 352-360 (2005).

[7] G. P. Jones IV, L. G. Pearlstine, and H. F. Percival. "An assessment of small unmanned aerial vehicles for wildlife research." Wildlife Society Bulletin 34(3), 750-758 (2006).

[8] J. A. Barnard. "Use of unmanned air vehicles in oil, gas and mineral exploration activities." In "AUVSI Unmanned Systems North America," (2010). 
[9] R. M. Caron. Aeromagnetic Surveying Using a Simulated Unmanned Aircraft System. Master's thesis, Carleton University (2011).

[10] R. Verbickas. Convolutional Neural Network-based Vision Systems for Unmanned Aerial Vehicles. Master's thesis, Carleton University (2013).

[11] L. A. Loeff and A. H. Soni. "An algorithm for computer guidance of a manipulator in between obstacles." Transactions of the ASME. Series B, Journal of Engineering for Industry 97(3), 836-842 (1975).

[12] L. Marce, M. Julliere, and H. Place. "Strategy of obstacle avoidance for a mobile robot." RAIRO Automatique 15(1), 5-18 (1981).

[13] K. L. Doty and S. Govindaraj. "Robot obstacle detection and avoidance determined by actuator torques and joint positions." In "Conference Proceedings of IEEE Southeastcon '82," pages 470-473. IEEE (1982).

[14] B. L. Chapman and N. Perreira. "Algorithm for intelligent control of a robot manipulator." In "Proceedings of the International MOTORCON Conference," pages 334-344. Intertec Communications Inc, Orlando, FL, USA (1983).

[15] O. Khatib. "Real-Time Obstacle Avoidance for Manipulators and Mobile Robots." The International Journal of Robotics Research 5(1), 90-98. ISSN 0278-3649 (1986).

[16] M. Hwangbo, J. Kuffner, and T. Kanade. "Efficient two-phase 3D motion planning for small fixed-wing UAVs." In "IEEE International Conference on Robotics and Automation," pages 1035-1041. Roma, Italy (2007).

[17] J. Amin, J. Boskovic, and R. Mehra. "A fast and efficient approach to path planning for unmanned vehicles." In "AIAA Guidance, Navigation, and Control Conference and Exhibit," AIAA 2006-6103. Keystone, Colorado (2006).

[18] J. Saunders, B. Call, and A. Curtis. "Static and dynamic obstacle avoidance in miniature air vehicles." In "Infotech@Aerospace," AIAA 2005-6950. Arlington, Virginia (2005).

[19] G. Fasano, D. Accardo, A. Moccia, C. Carbone, U. Ciniglio, F. Corraro, and S. Luongo. "Multi-Sensor based Fully Autonomous Non-Cooperative Collision Avoidance System for UAVs." Journal of Aerospace Computing, Information, and Communication 5, 338-360 (2008). 
[20] A. Smith, D. Coulter, and C. Jones. "UAS collision encounter modeling and avoidance algorithm development for simulating collision avoidance." In "AIAA Modeling and Simulation Technologies Conference and Exhibit," AIAA 20087043. Honolulu, Hawaii (2008).

[21] P. Angelov, C. D. Bocaniala, C. Xideas, C. Patchett, D. Ansell, M. Everett, and G. Leng. "A passive approach to autonomous collision detection and avoidance." In "Tenth International Conference on Computer Modeling and Simulation," pages 64-69. IEEE Computer Society (2008).

[22] L. Peng and Y. Lin. "A closed-form solution of horizontal maneuver to collision avoidance system for UAVs." In "Control and Decision Conference (CCDC)," pages 4416-4421. IEEE (2010).

[23] A. Tsourdos, B. White, and M. Shanmugavel. Cooperative path planning of unmanned aerial vehicles. American Institute of Aeronautics and Astronautics ; Wiley, West Sussex, U.K. ISBN 9781600867798 (2011).

[24] S. M. LaValle. Planning Algorithms. Cambridge University Press. ISBN 9780521862059 (2006).

[25] P. Chandler, S. Rasmussen, and M. Pachter. "UAV cooperative path planning." In "AIAA Guidance, Navigation and Control Conference," American Institute of Aeronautics and Astronautics (2000).

[26] A. Mujumdar and R. Padhi. "Evolving philosophies on autonomous obstacle/collision avoidance of unmanned aerial vehicles." Journal of Aerospace Computing, Information, and Communication 8, 17-41 (2011).

[27] S. M. Lavalle. "Rapidly-exploring random trees: A new tool for path planning." Technical report, Department of computer Science, Iowa State University, Ames, IA, USA (1998).

[28] S. LaValle and J. Kuffner. "Randomized kinodynamic planning." The International Journal of Robotics Research 20(5), 378-400 (2001).

[29] S. Griffiths and J. Saunders. "Maximizing miniature aerial vehicles." IEEE Robotics $\mathcal{G}$ Automation Magazine (September) (2006).

[30] P. Khosla and R. Volpe. "Superquadric artificial potentials for obstacle avoidance and approach." In "Proceedings. 1988 IEEE International Conference on Robotics and Automation," pages 1778-1784. IEEE Comput. Soc. Press (1988). 
[31] S. Ge and Y. Cui. "New potential functions for mobile robot path planning." IEEE Transactions on Robotics and Automation 16(5), 615-620 (2000).

[32] S. Ge and Y. Cui. "Dynamic motion planning for mobile robots using potential field method." Autonomous Robots 13(3), 207-222 (2002).

[33] J.-O. Kim and P. Khosla. "Real-time obstacle avoidance using harmonic potential functions." IEEE Transactions on Robotics and Automation 8(3), 338-349 (1992).

[34] S. Waydo and R. Murray. "Vehicle motion planning using stream functions." In "Proceedings of the 2003 IEEE International Conference on Robotics and Automation," pages 2484-2491. IEEE, Taipei, Taiwan (2003).

[35] F. Fahimi, C. Nataraj, and H. Ashrafiuon. "Real-time obstacle avoidance for multiple mobile robots." Robotica 27(02), 189-198 (2008).

[36] F. Fahimi, H. Ashrafiuon, and C. Nataraj. "Obstacle avoidance for spatial hyperredundant manipulators using harmonic potential functions and the mode shape technique." Journal of Robotic Systems 20(1), 23-33 (2003).

[37] J. Richalet. "Industrial applications of model based predictive control." Automatica 29(5), 1251-1274 (1993).

[38] D. Shim and S. Sastry. "An evasive maneuvering algorithm for UAVs in see-andavoid situations." In "Proceedings of the 2007 American Control Conference," pages 3886-3891. IEEE, New York City, USA (2007).

[39] D. Shim, H. Chung, H. Kim, and S. Sastry. "Autonomous exploration in unknown urban environments for unmanned aerial vehicles." In "Proc. AIAA GN\&C Conference," (2005).

[40] J. D. Anderson. Introduction to Flight. McGraw-Hill, New York, third edition. ISBN 0070016410 : (1989).

[41] C. R. Chester. Techniques in partial differential equations. McGraw-Hill, New York (1971).

[42] S. J. Axler, P. Bourdon, and W. Ramey. Harmonic function theory. Springer, New York, second edition. ISBN 0387952187 (2001).

[43] I. H. Shames. Mechanics of fluids. McGraw-Hill, New York, second edition. ISBN 0070563853 (1982). 
[44] J. D. Anderson. Fundamentals of aerodynamics. McGraw-Hill, Boston, MA, fourth edition. ISBN 0072950463 (2007).

[45] J. Stewart. Multivariable Calculus. Thomson Brooks/Cole, Belmont, sixth edition. ISBN 9780495011637 (2008).

[46] Dictionary of Scientific and Technical Terms. McGraw-Hill, New York, sixth edition (2003).

[47] I. Gradshteyn and I. Ryzhik. Table of Integrals, Series, and Products. Elsevier, Amsterdam, seventh edition. ISBN 978-0-12-373637-6 (2007).

[48] Museum of "Insitu Aerosonde Laima." http://www.museumofflight.org/aircraft/insitu-areosonde-laima. Accessed: 12 November 2012.

[49] Unmanned Dynamics, Hood River, OR. AeroSim aeronautical simulation blockset Version 1.2 Users's Guide.

[50] S. Kurnaz, O. Cetin, and O. Kaynak. "Fuzzy Logic Based Approach to Design of Flight Control and Navigation Tasks for Autonomous Unmanned Aerial Vehicles." Journal of Intelligent and Robotic Systems 54(1-3), 229-244. ISSN 0921-0296 (2008).

[51] MicroPilot Autopilot Installation \& Operation. Stony Mountain, MB, 20111207 edition (2011).

[52] H. Chao, Y. Cao, and Y. Chen. "Autopilots for small unmanned aerial vehicles: A survey." International Journal of Control, Automation and Systems 8(1), 36-44 (2010).

[53] M. Kumon, Y. Udo, H. Michihira, M. Nagata, I. Mizumoto, and Z. Iwai. "Autopilot System for Kiteplane." IEEE/ASME Transactions on Mechatronics 11(5), 615-624 (2006).

[54] E. Johnson and S. Kannan. "Adaptive flight control for an autonomous unmanned helicopter." In "AIAA Guidance, Navigation, and Control Conference and Exhibit," Number August in AIAA 2002-4439. Monterey, California (2002).

[55] H. Kim and D. Shim. "A flight control system for aerial robots: algorithms and experiments." Control Engineering Practice 11 (2003). 


\section{Appendix A}

\section{The $\delta$-Region}

In Section 3.5.2.2, it was mentioned that pathlines that are within the $\delta$-region of an obstacle are skipped. In Figures A.1 to A.5 the $R_{m i n}-h$ graph is plotted for different values of $\delta$. It can be seen that for smaller values of $\delta$, longer wedge lengths are required. The requirement for longer wedges comes from the pathlines whose starting points are within the $\delta$-region. However, the same long wedge length has to be used for pathlines with starting points outside of the $\delta$-region.

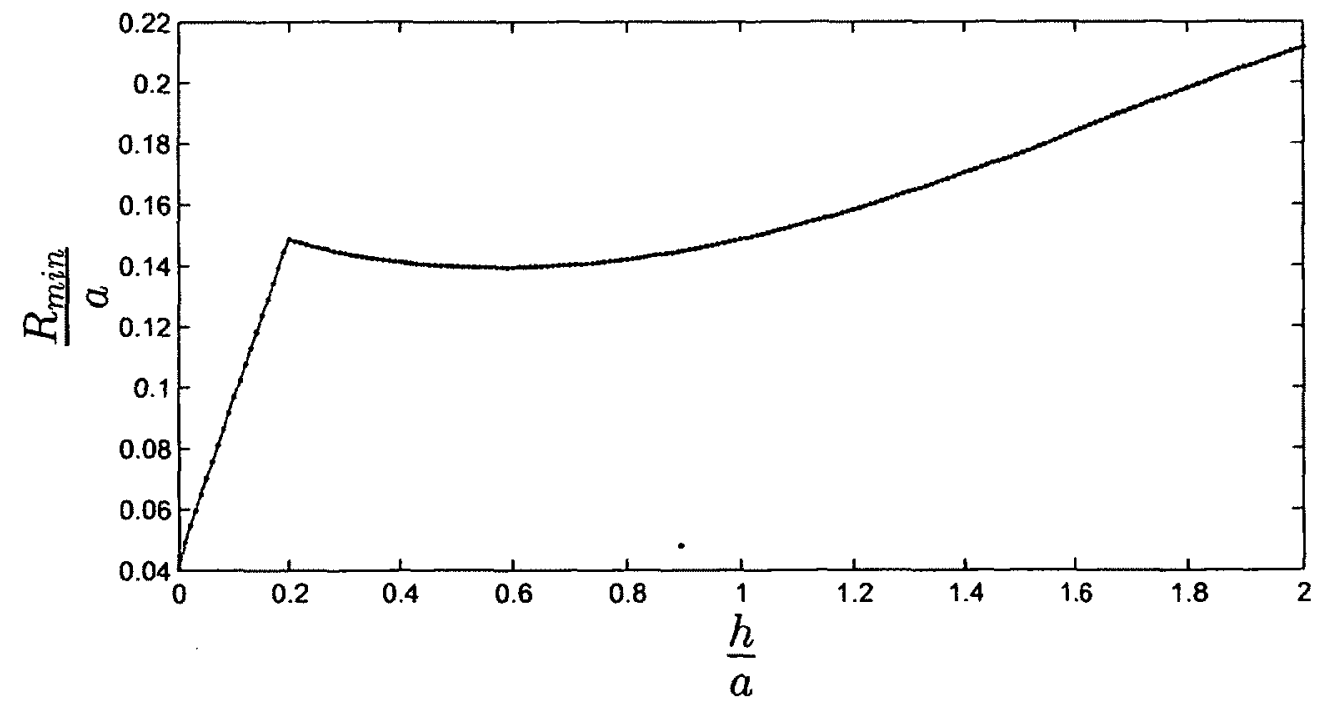

Figure A.1: Minimum radius of curvature for different wedge lengths for $\delta=0.02$ 


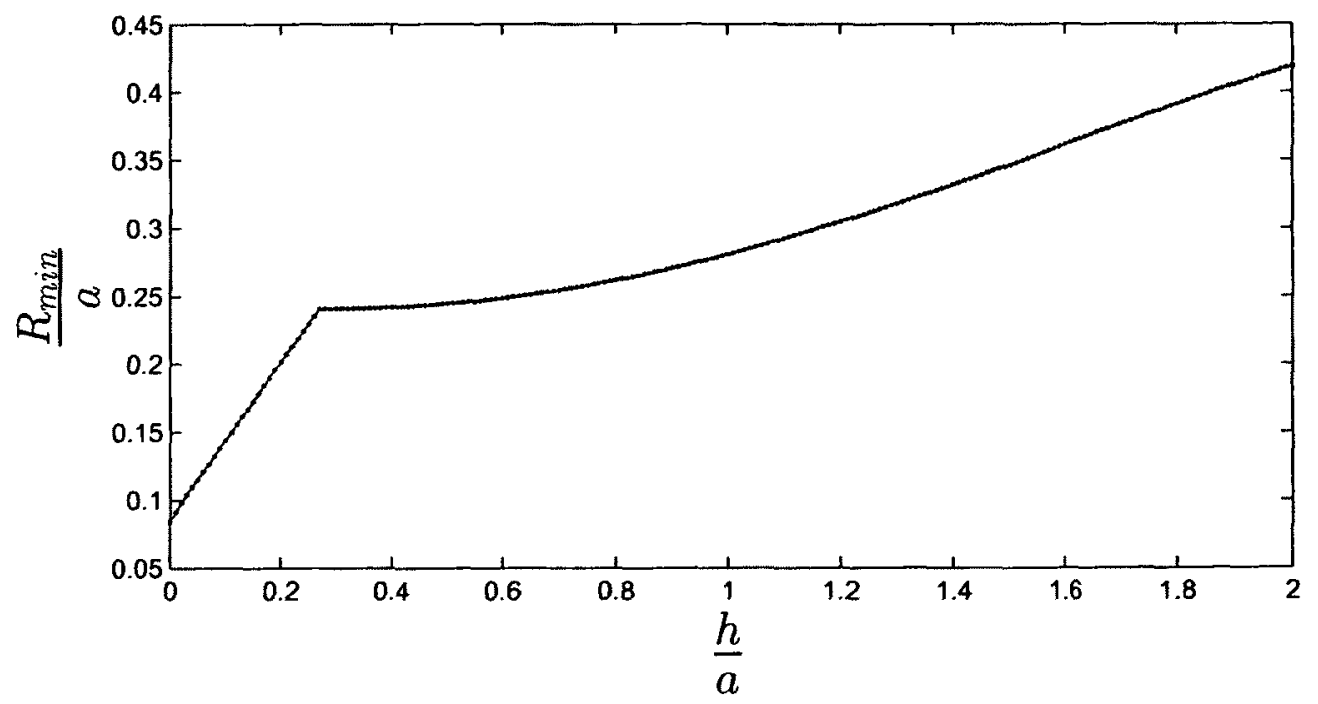

Figure A.2: Minimum radius of curvature for different wedge lengths for $\delta=0.04$

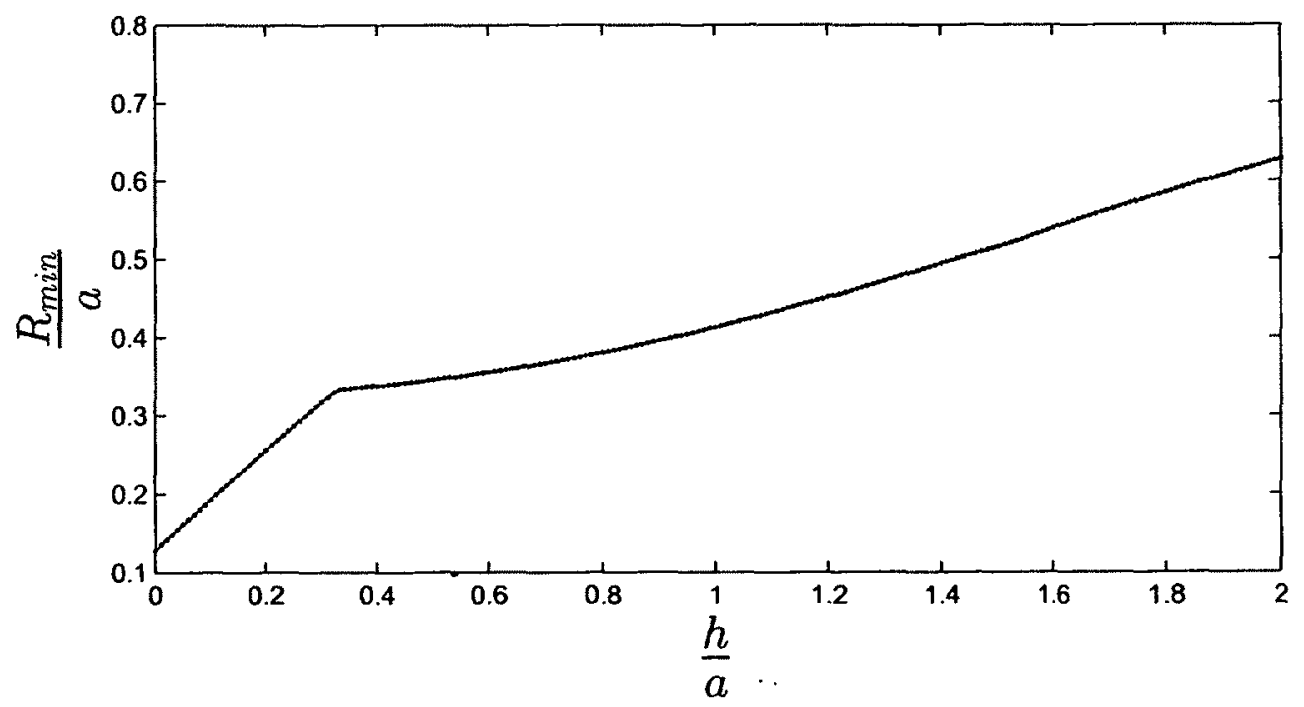

Figure A.3: Minimum radius of curvature for different wedge lengths for $\delta=0.06$ 


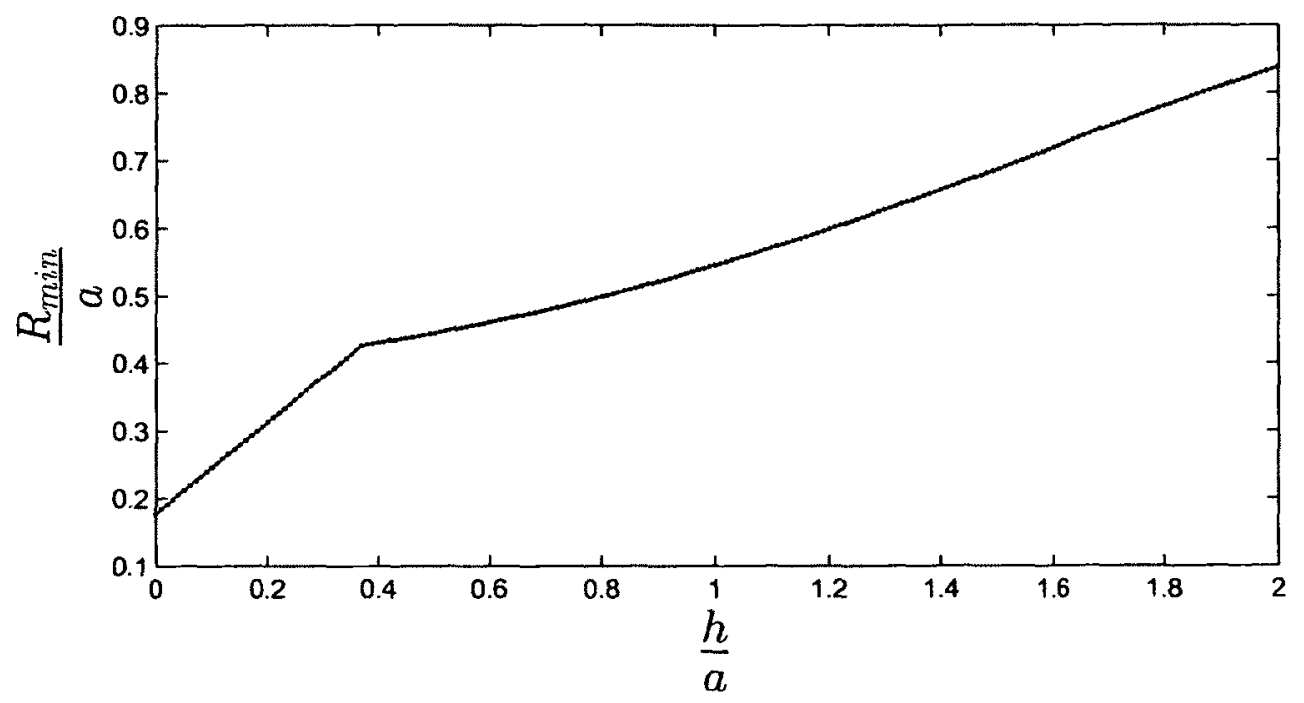

Figure A.4: Minimum radius of curvature for different wedge lengths for $\delta=0.08$

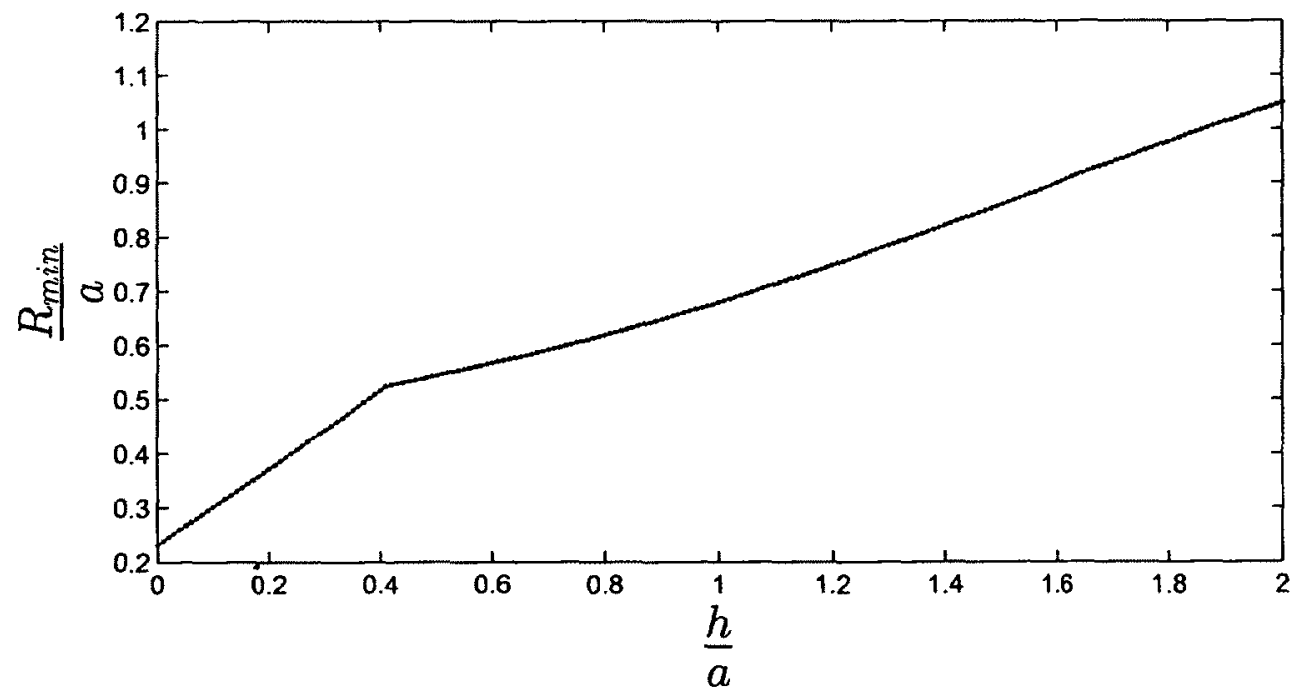

Figure A.5: Minimum radius of curvature for different wedge lengths for $\delta=0.1$ 


\begin{tabular}{|c|c|}
\hline$R_{\min }=31.13 m, a=50 m$ & $h(m)$ \\
\hline$\delta=0.02$ & 434.11 \\
\hline$\delta=0.04$ & 177.03 \\
\hline$\delta=0.06$ & 98.67 \\
\hline$\delta=0.08$ & 64.11 \\
\hline$\delta=0.1$ & 40.66 \\
\hline
\end{tabular}

Table A.1: Different wedge length required for a specific scenario based on the choice of $\delta$

In Table A.1, the wedge length for a specific scenario is interpolated from Figures A.1 to A.5 for different values of $\delta$. In the scenario used $R_{\min }=31.13 \mathrm{~m}$ and $a=50 m$.

However, using higher values for $\delta$ will result the aircraft being in the $\delta$-region more often, thus requiring more obstacle resizing. Therefore, in this thesis the value of $\delta$ is limited to 0.1 . 


\section{Appendix B}

In this thesis, obstacle avoidance trajectories for a fixed-wing aircraft are obtained by considering potential fluid flow around the obstacle. A pathline is a continuously differentiable curve in space, which yields the path along which a fluid particle flows around an obstacle (for any particle with initial condition on that particular pathline). As explained in Chapter 3, a pathline provides a suitable trajectory for an aircraft to follow in order to avoid an obstacle.

\section{B.1 Scaling of the Velocity Field}

It will now be shown that a pathline is independent of the magnitude of the velocity field.

Let $r(p):[a, b] \rightarrow \mathbb{R}^{3}$ be a pathline, and parameterize it by some variable $p \in \mathbb{R}$ in some closed interval $a \leq p \leq b$. The method used in this thesis to generate a pathline is from the potential flow velocity field $V(r): \mathcal{D} \rightarrow \mathbb{R}^{3}$, defined on some domain $\mathcal{D} \subset \mathbb{R}^{3}$. For the velocity fields given in Section 3.4, we may take the domain to be $\mathcal{D}=\mathbb{R}^{3} / \mathcal{O}$, where $\mathcal{O} \subset \mathbb{R}^{3}$ is a closed set defined by the obstacle. Then, it is readily seen that the velocity field $\mathrm{V}(\mathbf{r})$ given in Section 3.4 is continuous on the domain $\mathcal{D}$. Furthermore, since $\mathbf{V}(\mathbf{r}) \cdot \hat{\mathbf{n}}(\mathbf{r})=\mathbf{0}$ on the boundary of the obstacle $\partial \mathcal{O}$, where $\hat{\mathbf{n}}(\mathbf{r})$ is the unit outward pointing normal at each given point on the obstacle boundary, it must be that any pathline with initial condition $\mathbf{r}(a) \in \mathcal{D}$ satisfies $\mathbf{r}(p) \in \mathcal{D}$ for all 
$p \in[a, b]$ (that is, if it starts in $\mathcal{D}$, it must stay in $\mathcal{D}$ ). Finally, it is to be noted that the velocity fields given in Section 3.4 satisfy $V(r) \neq 0$ for all $\mathbf{r} \in \mathcal{D}$.

If the pathline parameterization is time $(p=t)$, then the pathline $\mathbf{r}(t)$ is an integral curve of the first order differential equation

$$
\frac{d \mathbf{r}}{d t}=\mathbf{V}(\mathbf{r})
$$

with initial condition $\mathbf{r}(a) \in \mathcal{D}$ given. Since $d \mathbf{r} / d t \neq \mathbf{0}$ for all $t \in[a, b]$ (because $\mathbf{V}(\mathbf{r}) \neq \mathbf{0}$ on $\mathcal{D})$, the pathline may equivalently be parameterized by its arc-length, $s$, measured along the pathline from the initial condition $\mathbf{r}(a)$. That is, the pathline may be given by $\overline{\mathbf{r}}(s):\left[0, s_{\max }\right] \rightarrow \mathbb{R}^{3}$, where $\overline{\mathbf{r}}(s)=\mathbf{r}(t(s))$, and $s_{\max }$ is the length of the pathline. It is a property of the arc-length that

$$
\frac{d s}{d t}=\left|\frac{d \mathbf{r}}{d t}\right|
$$

Therefore, from (B.1), it is readily found that the pathline is also an integral curve of

$$
\frac{d \overline{\mathbf{r}}}{d s}=\frac{1}{|\mathbf{V}(\overline{\mathbf{r}})|} \mathbf{V}(\overline{\mathbf{r}})
$$

with initial condition $\overline{\mathbf{r}}(0)=\mathbf{r}(a)$.

We are free to parameterize $s$ by any other parameterization $\tau$ (which can be thought of as a different time-scale). In particular, let $g(s) \geq \epsilon>0$ be a strictly positive continuous function, and suppose that $s$ is the solution of the first order differential equation

$$
\frac{d s}{d \tau}=g(s)
$$

on an appropriate interval $\tau \in[c, d]$ with initial condition $s(c)=0$. Note that $s(\tau)$ is a strictly increasing function, and the upper limit of the interval $d$ is set such 
that $s(d)=s_{\max }$ (which can be done since $d s / d \tau \geq \epsilon>0$ ). Then, the pathline is equivalently represented by $\hat{\mathbf{r}}(\tau):[c, d] \rightarrow \mathbb{R}^{3}$, where $\hat{\mathbf{r}}(\tau)=\overrightarrow{\mathbf{r}}(s(\tau))$.

Now, let $\ddot{a}(\mathbf{r})>0$ be a strictly positive continuous function on $\mathcal{D}$, and let $g(s)$ in (B.3) be given by

$$
g(s)=a(\overline{\mathbf{r}}(s))|\mathbf{V}(\overline{\mathbf{r}}(s))|
$$

Clearly, $g(s)>0$ for $s \in\left[0, s_{\max }\right]$, and is continuous. Note that since the pathline $\overline{\mathbf{r}}(s)$ represents a closed and bounded set on the interval $\left[0, s_{\max }\right]$, we can readily find an $\epsilon>0$ such that $g(s) \geq \epsilon$ (just take $\epsilon=\min _{s \in\left[0, s_{\text {max }}\right\}}\{g(s)\}>0$ ). Finally, by combining (B.3) and (B.4) with (B.2), it is readily found that the pathline is also an integral curve of

$$
\frac{d \hat{\mathbf{r}}}{d \tau}=a(\hat{\mathbf{r}}) \mathbf{V}(\hat{\mathbf{r}})
$$

with initial condition $\hat{\mathbf{r}}(c)=\mathbf{r}(a)$.

To conclude, it has been shown that pathlines are integral curves of the differential equations (B.1), (B.2) and (B.5). The variables used to parameterize the pathlines $(t, s, \tau)$, can simply be viewed as variables of integration in (B.1), (B.2) and (B.5), and may be interpreted as different time-scales. Therefore, the right-hand sides of (B.1), (B.2) and (B.5) may all be viewed as velocity fields leading to the same pathlines. As such it may be concluded that a pathline is completely determined by the direction of the velocity field at each point. The magnitude simply changes the temporal nature with which the pathline is traversed. This justifies why, at each point in an aircraft's trajectory, the direction of the local velocity field may be used to provide either a commanded course or commanded path angle (without consideration of the aircraft speed). With perfect tracking, the aircraft will then follow a fluid pathline. 


\section{B.2 Scaling of the Coordinate System}

Consider a velocity field $\mathbf{V}(\mathbf{r})$. As shown in the previous section, the resulting pathlines are integral curves of (B.1). Let $\mathbf{r}(t)$ be a pathline of corresponding to (B.1) with initial condition $\mathbf{r}(a)$, and consider a scaling of the coordinates $\mathbf{r}_{s}=\alpha \mathbf{r}$. In particular, consider the curve $\mathbf{r}_{s}(t)=\alpha \mathbf{r}(t)$. Then, it is readily found that $\mathbf{r}_{s}(t)$ is an integral curve of

$$
\frac{d \mathbf{r}_{s}}{d t}=\alpha \mathbf{V}\left(\mathbf{r}_{s} / \alpha\right)
$$

with initial condition $\mathbf{r}_{s}(a)=\alpha \mathbf{r}(a)$. As seen in the previous section, the pathlines are unaffected by scaling of the velocity field. Hence, $\mathbf{r}_{s}(t)$ is equivalently an integral curve of

$$
\frac{d \mathbf{r}_{s}}{d t}=\mathbf{V}\left(\mathbf{r}_{s} / \alpha\right)
$$

with initial condition $\mathbf{r}_{s}(a)=\alpha \mathbf{r}(a)$, and therefore is a pathline generated by the velocity field $\mathbf{V}\left(\mathbf{r}_{s} / \alpha\right)$. That is, scaling of the coordinate system results in the same scaling of the pathlines.

\section{B.3 Scaling an Obstacle}

In this section, it is shown that if an obstacle is scaled, the shapes of pathlines do not change (the pathlines of the scaled square are geometrically similar to that of the unit square).

Say that the unit square obstacle was scaled by a factor of $\alpha>0$. Therefore 
examining (3.53) suggests ${ }^{1}$

$$
\begin{aligned}
& A_{s}=\alpha A \\
& B_{s}=\alpha^{2} B \\
& C_{s}=C \\
& D_{s}=\alpha D
\end{aligned}
$$

Furthermore it is clear that

$$
S_{j_{s}}=\alpha S_{j}
$$

By substituting the values of (B.8) and (B.9) into (3.54), it can be seen that $I_{i, j}$ will not change. Consequently, from (3.42) it is concluded that the panel strengths will remain constant, i.e.

$$
\lambda_{i_{s}}=\lambda_{i}
$$

Now, set $x_{s}=\alpha x, y_{s}=\alpha y$. Then, from (3.56) to (3.58), (3.48) and (3.49) it can be concluded that

$$
\begin{aligned}
& J_{x j_{s}}\left(x_{s}, y_{s}\right)=J_{x j}(x, y)=J_{x j}\left(x_{s} / \alpha, y_{s} / \alpha\right) \\
& J_{y j_{s}}\left(x_{s}, y_{s}\right)=J_{y j}(x, y)=J_{y j}\left(x_{s} / \alpha, y_{s} / \alpha\right) .
\end{aligned}
$$

Finally from (3.46), (3.47), (B.10) and (B.11) it is obvious that the velocity field is given by

$$
\begin{aligned}
& V_{x_{s}}\left(x_{s}, y_{s}\right)=V_{x}\left(x_{s} / \alpha, y_{s} / \alpha\right) \\
& V_{y_{s}}\left(x_{s}, y_{s}\right)=V_{y}\left(x_{s} / \alpha, y_{s} / \alpha\right) .
\end{aligned}
$$

As shown previously, this means that if an obstacle is scaled by a factor of $\alpha$, all

\footnotetext{
${ }^{1}$ The $s$ subscript is used for the scaled obstacle
} 
pathlines will be scaled by $\alpha$ as well. Consequently,

$$
R_{\min }=\alpha R_{\min }
$$

\section{B.4 Effect of $V_{\infty}$}

In this section it is shown that in the absence of a goal the magnitude of $V_{\infty}$ does not affect the pathlines.

Let

$$
V_{\infty}^{\prime}=\beta V_{\infty}
$$

From (3.42)

$$
\lambda_{i}^{\prime}=\beta \lambda_{i}
$$

and from $(3.46),(3.47)$

$$
\begin{aligned}
& V_{x}^{\prime}=\beta V_{x} \\
& V_{y}^{\prime}=\beta V_{y}
\end{aligned}
$$

From (B.16) it is concluded that even though, the magnitude of the velocity changes, its direction remains unchanged. Hence, as shown previously, the pathlines do not change. 


\section{Appendix C}

\section{Definitions}

Course: The course is the direction of travel expressed as an angle in the horizontal plane between a reference line and a tangent to the flight path.

Heading: The heading is the direction that the aircraft's nose is pointing.

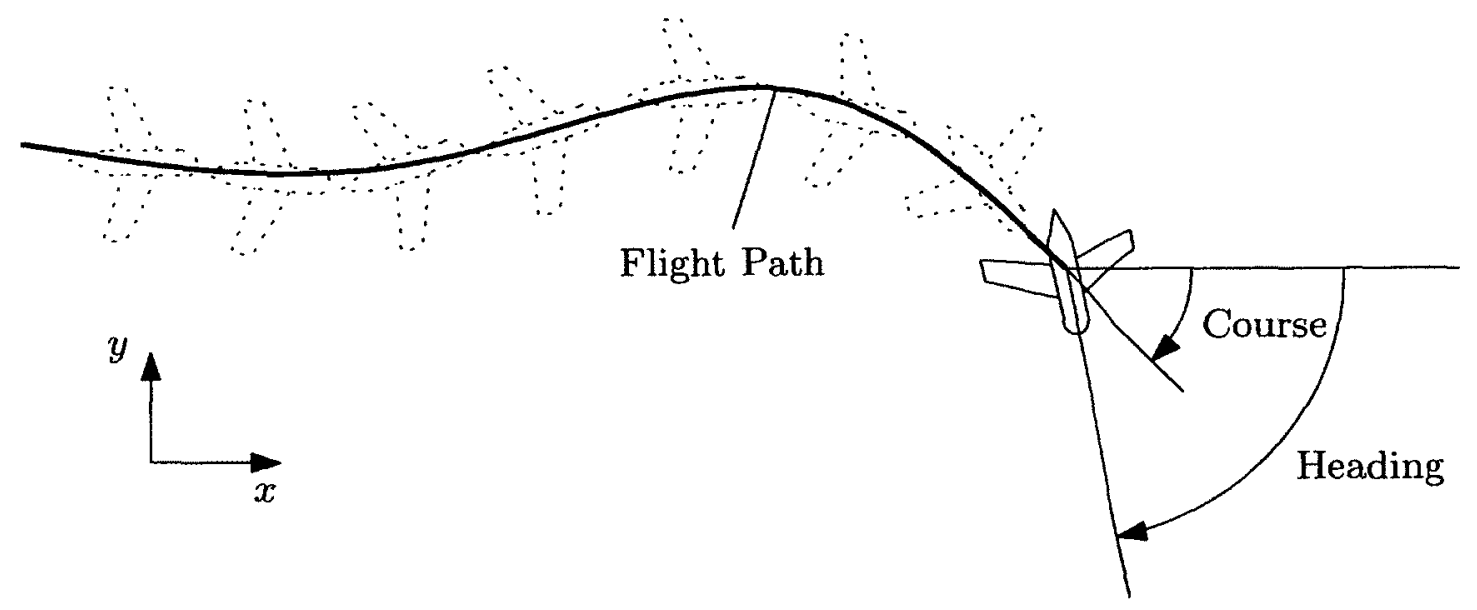

Figure C.1: Aircraft course and heading

Path Angle: The path angle is the angle between the horizontal and a tangent to the flight path. 


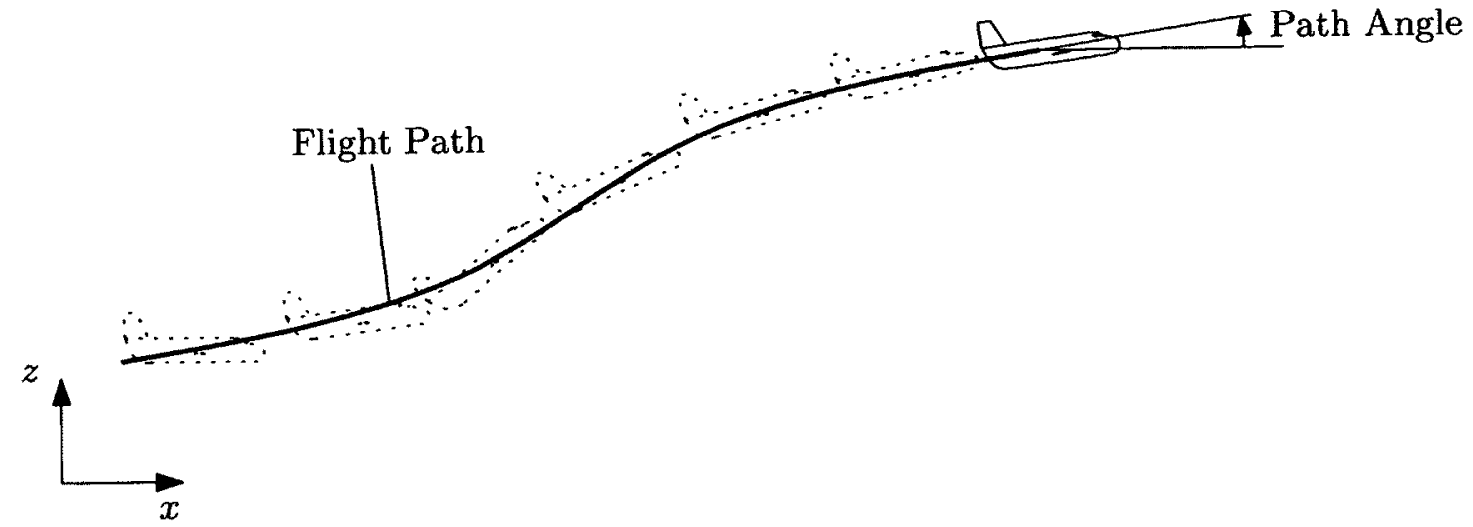

Figure C.2: Aircraft path angle 